\title{
Anwendung und Wirkungsweise des I.K. an der Hand von Krankengeschichten aus der Carl Spenglerschen Praxis.
}

\section{Von}

\author{
Dr. R. Benöhr, Assistent von Dr. C. Spengler
}

und A. Hoffmann in Davos, jetzt Spezialist für Lungenleiden in Mainz.

Mit 9 Kurventafeln.

In folgendem veröffentlichen wir aus der Carl Spenglerschen Praxis $130^{\circ}$ Krankengeschichten, die uns zum Verständnis der Anwendung und Wirkungsweise des I.K. Charakteristisches zu bieten scheinen. Dabei haben wir uns von folgenden Gesichtspunkten leiten lassen: Initialfälle, deren Heilung als unschwierig nichts Charakteristisches bietet, haben wir ausgeschieden; vorwiegend bringen wir fiebernde Fälle, um die verschiedenen Arten der Entfieberung zu zeigen; dann Fälle, die längere Zeit erfolglos klimatisch oder spezifisch in Behandlung waren; ferner solche, die durch ihre besondere Schwere und Hartnäckigkeit Schwierigkeiten boten. Aus diesen Gruppen haben wir die Repräsentanten ausgesucht, weil es aus Raummangel nicht möglich ist, sämtliche Krankengeschichten zu veröffentlichen. Klassifiziert sind sie nach der Carl S penglerschen KlassenstadienEinteilung, die sich im Laufe der Jahre durch ihre Einfachheit und Übersichtlichkeit sehr bewährt hat. Des Zusammenhangs halber sei dieselbe hier wiederlıolt:

I. Klasse. Nichtfiebernde.

II. Klasse. Fiebernde.

1. Stadium.

Inaktive geschlossene Tuberkulose mit beliebigem physikalischem Befund geschlossener Formen. Tuberkulinre. aktion.
Aktive, geschlossene Tuberkulose mit leichtem tuberkulösem Fieber, sonst wie Stadium 1, Klasse I. 
2. Stadinm.

Inaktive Initialphthise ohne nachweisbare Kavernen, mit tuberkulösen Sputummengen bis $20 \mathrm{ccm}$ in 24 Stunden. Passive Begleitinfektion.
Aktive Initialphthise mit leichtem oder mittlerem tuberkulösem Fieber, sonst wie Stadium 2, Klasse I. Leichto tuberkulöse Larynx- und Pleurakomplikationen. Aktive Begleit- und subaktive Mischinfektion.

3. Stadium.

a) Inaktive kavernöse Phthise mit mehr als $20 \mathrm{ccm}$ tuberkulösen Sputams in 24 Stunden. Eventuell leichte tuberkulöse Komplikationen; Larynx, Pleura, Darm. Passive Begleit- und passive Mischinfektion.

b) Nur durch operative Eingriffe schlussfähige, starrwandige Höhlen. a) Aktive, kavernöse Phthise mit tuberkulösem oder subkutanem Mischfieber (lokalisierte Mischinfektion). Event. tuberkulöse Komplikationen, Darm,Blase, Pleura, Larynx.

b) Nieren- und andere schwerste Komplikationen. Diffuse Mischphthise, käsige Pneumonie. Akute disseminierte und miliare Tuberkulose.

Bezüglich der Bazillenbefunde bedienen wir uns der C. Spengle rschen Bazillenskala, die hier auch kurz wiederholt sei:

0-1. T.B. äusserst selten; im ganzen Präparat nur vereinzelt.

1. Vorhanden. Spärliche Bazillen und nicht in jedem Gesichtsfeld.

1-2. Ziemlich zahlreich; in jedem Gesichtsfeld weniger als 12.

2. Zahlreich. In jedem Gesichtsfeld durchschnittlich 12 .

2-3. Sehr zahlreich; in jedem Gesichtsfeld mehr als 12, aber noch zählbar.

3. In grossen Mengen; nicht mehr zäblbar. $3 \rightarrow \infty$ wie in Reinkultur.

Die Erfolge der I.K.-Behandlung werden durch die beifolgenden Krankengeschichten deutlich erläutert. Die Wirkungen des Mittels fassen wir nochmals zusammen:

\section{Antitoxische Wirkung.}

Die ausgesprochene antitoxische Wirkung besteht in der Beseitigung der toxischen Erscheinurigen, wie Fieber, Schlaflosigkeit, nervöse Störungen, Nachtschweisse usw., und als deren Folge Hebung des Allgemeinbefindens, Appetit- und Gewichtszunahme. Dadurch wird auch die Leistungsfähigkeit gesteigert.

\section{Bakteriolytisch-anatomische Wirkung.}

Die Iytischen Elemente des I.K. greifen die Bakterien in ihren Hüllen an, lösen die Hülle auf und bringen das Stäbchen zum Zer- 
fall und splittern es. Dadurch werden die Ekto- und Endotoxine gelöst, die dann ihrerseits ihre aktive tuberkulinisierende Wirkung ausüben (Autotuberkulinisierung). Die Abnahme der Bazillen geht in verschiedener Weise vor sich. Entweder die Bazillen nehmen unter der I.K.-Behandlung kontinuierlich ab, um dann definitiv zu verschwinden. Oder aber die Bazillenzahl vermindert sich rasch, in kurzer Zeit von 2-3 der Spenglerschen Skala auf 0-1, danach ein- oder mehrmaliger Massenausstoss von Bazillen, wieder 0-1, wieder Massenausstoss, und dann gänz lich es Verschwinden („Pendelabnahme").

Dieses Bild der Bazillenabnahme kann man natürlich nur durch $\mathrm{h} \ddot{u} \mathbf{u f i g e}$ Untersuchungen des Sputums gewinnen, wie sie in Spenglers Institut an der Tagesordnung sind. Man erlangt nicht durch wenige sporadische Untersuchungen, sondern durch fortlaufende, bei jedem Patienten in kurzen Zwischenräumen vorgenommene Sputumanalysen eine ständige Kontrolle über das Verhalten der Bakterien. Die Durchschnittszahl der Sputumuntersuchungen in Spenglers Institut beträgt für den einzelnen Fall 20--30, von einem Fall liegen sogar 96 Untersuchungen vor. Besonders häufig sind die Untersuchungen im Stadium der Abnahme und im bazillenfreien Stadium. Neben diesen mikroskopischen Untersuchungen finden noch häufig kulturelle statt, um die Entwickelung von Stäbchen aus eventuell noch vorhandenen lebensfähigen Splittern (Sporen) zu konstatieren.

Während nun die kombinierte antitoxische und lytische Wirkung die Anwendung von I.K. für Arzt und Patienten viel angenehmer macht, so hat I.K., wie sich aus der klinischen Beobachtung ergibt, auf den ganzen Heilungsprozess der Tuberkulose eine viel promptere Wirkung, als die übrigen Spezifika. Vor allem auffallend ist das rasche Schwinden der katarrhalischen Erscheinungen, auch bei Fällen, die vorher ohne Erfolg klimatisch und anderweitig spezifisch behandelt worden sind (s. z. B. Nr. 3, 7, 9, 13, 20, 23, 37, 38, 39, 40, 42, $61,64,67,68,91,107,108,118,119,126)$.

\section{Wirkung auf die Temperatur.}

Besondere Beachtung verdient die Wirkung des I.K. auf die Temperatur des Kranken. Bei einer Beihe von Fällen sieht man bei der Anwendung des Mittels, auch schon bei kleinsten Dosen, eine prompte Entfieberung (s. Kurve Nr. 2). Bei anderen wird die Entfieberung erst bei längerer Behandlung erreicht (s. Kurve Nr. 6); solche sind besonders Fälle mit Pleura-Komplikationen.

Allerdings sind dieser Entfieberung zuweilen einige Temperaturerhöhungen vorangegangen, die als lytische aufzufassen sind. Diese 
Temperaturerhöhungen erstrecken sich für gewöhnlich nur auf einige Stunden oder Tage.

Nach Ablauf der febrilen Erscheinungen sieht man oft die Temperatur völlig normiert (Immunkurve), die zuvor mehr oder minder zahlreichen Bazillen vermindert oder verschwunden, den anatomischen Lungenbefund wesentlich gebessert. Aus oben Gesagtem ergibt sich als selbstverständlich, dass auch afebril in Behandlung gekommene Patienten im Verlauf derselben vorübergehend kleine febrile Reaktionen zeigen können (s. Kurve Nr. 10).

In der Lysis findet sich oft die Temperatur erhöht, andererseits macht die Lysis auch ihre Einwirkung anf das Lungengewebe geltend, wodurch vorübergehend die katarrhalischen Erscheinungen gesteigert erscheinen können. Wenn daher von mancher Seite behauptet wird, man habe unter I.K. Verschlimmerungen gesehen, so können wir dies nur auf eine Verkennung der oben geschilderten Wirkung des I.K. zurückführen.

\section{Anwendungsweise.}

Bezüglich der dem Präparat beigegebenen Anwendungsweise bemerken wir, dass dieselbe für den praktischen Arzt nur als Anleitung dienen soll. Die optimale Behandlung ist für jeden Fall durchaus individuell. Ein Schema lässt sich nicht angeben. Im allgemeinen empfiehlt es sich, die I.K.-Kur mit einer Injektion von 2-5 Teilstrichen der VII. - V. Verdünnung, d. h. $\frac{2-5}{10} \mathrm{ccm}$ einer zehnmillionen- bis hunderttausendfacben Verdünnung der Stammlösung zu beginnen. Nach der ersten Injektion wartet man zweckmässig 8-10 Tage und steigert dann in kürzeren Intervallen (3-6 Tage) die Dosis jedesmal um das Zehnfache, eventuell mehr, bis auf einen Teilstrich Originallösung (s. Kurve 2 und 3). Bei Anwendung des Originals kann man die Dosen in folgender Reihenfolge anwenden: 1 Teilstrich, 2, 5, 10 d. h. $1 \mathrm{ccm}$. Oft erweist es sich als zweckmässig, vom Original auf die Anfangsdosen zurückzugreifen und einen neuen Anstieg in rascherem Tempo vorzunehmen, um das Hundert- oder Tausendfache zum Original ansteigend. Die lytischen Wirkungen des Präparats werden bei diesem Vorgehen am meisten ausgenutzt. Sehr gut ist auch für viele Fälle das "retrograde" Vorgehen, d. h. man steigt, wie oben beschrieben, auf die Originallösung, und dann in gleichem Verhältnisse zurück auf die V.-VII. Verdünnung. Lytische Wirkungen bieten an sich kein Hindernis zur Weiterführung der Immunisierung. Man wird bei Reaktionen mit Rücksicht auf die im I.K. enthaltenen Antitoxine ruhig in der Behandlung fortschreiten können. Überwiegt 
trotzdem die lytische Wirkung die antitoxische, so wird man die Lysis oft durch eine Injektion (oder Einreibung) einer um das 1000 oder 10000 fachen Verdünnung koupieren können. .

Was von den Injektionen gesagt ist, gilt auch von den Einreibungen, wie oben erläutert ist (s. Kurve Nr. 11).

Bei Befolgung obiger Regeln wird ein Schaden für den Patienten niemals entstehen können, hingegen wird der in der I.K.-Therapie erfahrene Arzt durch individuelle Modifikation das für den jeweiligen Patienten therapeutische Optimum finden.

Was nun die von uns veröffentlichten Krankengeschichten betrifft, so möchten wir darauf hinweisen, dass - wie sich aus den Anamnesen ergibt - eine ganze Reihe von Patienten vor Eintritt in die I.K.-Behandlung bereits in Davos oder anderweitig klimatische oder spezifische Kuren ohne Erfolg durchgemacht baben. Viele unserer Fälle machen den Eindruck, geheilt zu sein. Trotzdem haben wir den Ausdruck "geheilt" absichtlich vermieden, weil sich die Richtigkeit einer solchen Behauptung erst nach einer oder mehreren etappenweise vorgenommenen Nachuntersuchungen oder Nachbehandlungen rechtfertigen lässt. Insbesondere verweisen wir auf die relativ kurzen Behandlungszeiten, innerhalb deren auch bei den schwersten Fällen, bei denen eine Heilung erst nach Jahren möglich ist, trotzdem eine auffallende Besserung erzielt wurde. Setzen doch auch in Davos die finanziellen Verhältnisse des Patienten dem Arzt oft ein "non possumus" entgegen.

Der Natur unseres Kurorts entsprechend kommen hier hauptsächlich nur Lungentuberkulose und deren Komplikationen in Behandlung. An anderen Organen lokalisierte Tuberkulosen haben wir hier in Davos selten. Immerhin verfügt Dr. C. Spengler über einige solcher Fälle, in denen I.K. ebenfalls eine günstige Wirkung ausübte. Zwei Lupusfälle wurden durch I.K. mit recht gutem Erfolg behandelt. Aus dem Tiefland berichtet uns Dr. Schmitt-Weingarten über die Heilung eines Falles von Lupus durch I.K.: an beiden Beinen bestanden zahlreiche Geschwüre, in deren Sekret Splitter nachgewiesen wurden. Ferner nennen wir unter anderem eine hier in Davos bereits längere Zeit ohne Erfolg behandelte Phlyktäne, die jetzt als vollständig geheilt $\mathrm{zu}$ betrachten ist; dann ein sehr ausgedehntes, hartnäckiges skrofulöses Ekzem bei einem hereditär belasteten anderthalbjährigen Kind, durch I.K.-Einreibungen geheilt. Der später geborene Bruder dieses Kindes kam mit gleichen Erscheinungen in die Behandlung und wurde ebenfalls geheilt. Hier seien auch die guten I.K.-Erfolge bei Kinderskrofulose erwähnt, die Dr. Selter 
und Dr. Gernsheim auf dem Kongress Niederrhein.-Westfäl. und süddeutscher Kinderärzte, Wiesbaden, April 1909, bekannt gaben.

Chirurgische Tuberkulosen kamen nicht in Behandlung. Hingegen wurde uns von mehreren Seiten über erfolgreiche Verwendung der I.K.-Therapie berichtet. Siehe z. B. die untenstehende Arbeit von Dr. Westphal.

Zum Schluss einige Bemerkungen zu unseren beigegebenen Temperaturkurven. Vorausgeschickt sei, dass wir der Raumerparnis halber bei einigen nur die Tagesminima und -Maxima aufgezeichnet haben. Im Gegensatz zu den I.K.-Injektionen sind die Einreibungen mit $\infty$ bezeichnet.

Kurve 1 (s. Krankengeschichte Nr. 35) bietet das Bild einer unruhigen, leicht toxisch beeinflussten Kurve, die nach einigen Wochen unter Verlust des Sputums in eine anhaltende Immunkurve übergent.

Kurve 2 (s. Krankengeschichte Nr. 111). Dieser Fall war bereits seit einiger Zeit fiebernd in Davos. Die sofort nach Einsetzen der I.K.-Therapie eingetretene Entfieberung ist daher nicht als eine klimatische anzusehen. Der Schluss der Kurve ist wieder als Immunkurve anzusprechen.

K urve 3 (s. Krankengeschichte Nr. 124). Die Kurve einer akut einsetzenden floriden Phthise (Lungen- und Darmtuberkulose) mit infauster Prognose. Man vergleiche damit den in $3^{1 / 2}$ Monaten erreichten Erfolg. (S. auch physikalischen Befund).

K urve 4 (s. Krankengeschichte Nr. 101). Gleichfalls ein schwerer durch I.K. in relativ kurzer Zeit wesentlich gebesserter Fall, der schon mehrere Monate vor Eintritt in die Behandlung hochfiebernd war. Patient ist noch in Behandlung. Die Temperaturerhöhung am 29. September ist als Ausdruck einer lytischen Reaktion anzusehen.

Kurve 5 (s. Krankengeschichte Nr. 96). Die anhaltende Wirkung des I.K. beweist der Auszug der Temperaturkurve nach 6 Monaten. Bezüglich der Schwere des Falles verweisen wir ganz besonders auf die Krankengeschichte.

Kurve 6 (s. Krankengeschichte Nr. 99) zeigt uns, dass man auch bei schwersten Fällen, die der Besserung hartnäckigen Widerstand entgegensetzen, schliesslich Erfolg haben wird. Auch hier zeigt die 11/2 Monate spätere Kurve die anhaltende Wirkung des I.K.

Kurve 7 (s. Krankengeschichte Nr. 17). Bei dieser 60jährigen Frau verschwanden die Tuberkelbazillen nach der ersten Injektion definitiv. 
Kurve 8 (s. Krankengeschichte Nr. 49). Tuberkelbazillen nach drei Injektionen definitiv verloren.

K u rve 9 (s. Krankengeschiclite Nr. 21). Tuberkelbazillen gleichfalls in kurzer Zeit definitiv verschwunden.

Kurve 10 (s. Krankengeschichte Nr. 53). Ein afebriler Fall, dessen Temperatur im Verlauf der Behandlung mehrere Erhöhungen (bis 38,3) aufweist, die als lytische Reaktionen aufzufassen sind. Im späteren Verlauf wird die Temperatur wieder durchaus ruhig und normiert. Man beachte auch den Sputumabfall!

Kurve 11 (s. Krankengeschichte Nr. 114). Behandlung fast nur mit Einreibungen; zum Schluss eine typische Immunkurve.

Wir glauben durch beigegebene Krankengeschichten den Beweis für die Wirksamkeit des I.K. erbracht zu haben. Mit den von Dr. C. Spengler erreichten Erfolgen decken sich die Erfolge zahlreicher anderer Ärzte. Die Misserfolge einzelner Ärzte auf das Mittel zurückzuführen, wäre angesichts des grossen positiven Materials ungerecht; es liegt vielmehr nahe, sie auf den Anwendenden selbst zurückzuführen. Zum Teil liegt es auch an der schiefen Beurteilung des Erreichten. So weisen z. B. die von Dr. Simon (Zeitschrift für Tuberkulose, Bd. XV, 1909) publizierten Fälle grösstenteils befriedigende Resultate auf; u. a. ist bei den meisten der behandelten Patienten die Leistungsfähigkeit wesentlich gehoben; den Gegenbeweis, dass diese Besserungen nicht auf I.K. zurïckzuführen sind, ist Dr. Simon schuldig geblieben. Das gleiche gilt von Dr. Kerlé und Dr. Weirauch. In übrigen weisen wir hier auf die Bemerkung von Dr. Hollos hin („Tuberkulöse Intoxikationen“, erschienen bei Franklintársulat, Budapet 1909): „Ich schreibe die ungünstigen Resultate eher der schablonenhaften, nicht individualisierenden Behandlung zu. Und wenn jemand wie Roepke, einfach I.K. als Kochsalzlösung betrachtet und auch so damit umgeht, so wird eine solche Behandlung dem Kranken eher schädlich als nützlich sein". Siehe auch Wallerstein: „Über I.K.“ Berliner Klinische Wochenschrift No. 14. 1910. 
Klasse I,

\begin{tabular}{|c|c|c|c|c|c|}
\hline \multirow{2}{*}{ Nr. } & \multirow{2}{*}{$\begin{array}{l}\text { Name, } \\
\text { Alter, } \\
\text { Beruf }\end{array}$} & \multirow{2}{*}{ Anamnese } & \multirow{2}{*}{$\begin{array}{c}\text { Kom- } \\
\text { plikationen }\end{array}$} & \multicolumn{2}{|c|}{ Lungenbefund } \\
\hline & & & & Eintritt & Entlassung \\
\hline 1 & $\begin{array}{l}\text { H., } \\
28 \text { J., } \\
\text { Fräulein }\end{array}$ & $\begin{array}{c}\text { Vor } 8 \text { Wochen } \\
\text { Hämoptoe ; Schlaf } \\
\text { Appetit schlecht }\end{array}$ & - & $\begin{array}{l}\text { Beide Oblpp. rel. } \\
\text { Dpf., rauhes vesiko- } \\
\text { bronchial. Atmen. } \\
\text { Rechte Spitze, bes. } \\
\text { H., vereinzeltes } \\
\text { Knarren }\end{array}$ & Keine Rhonchi mehr \\
\hline 2 & $\begin{array}{l}\mathrm{Z}, \\
51 \mathrm{~J} ., \\
\text { Kaufmann }\end{array}$ & $\begin{array}{l}\text { Seit mehreren } \\
\text { Wochen Husten }\end{array}$ & - & $\begin{array}{l}\text { R. Spitze vesiko- } \\
\text { bronchial, einzelne } \\
\text { Rhonchi u. Rauhig- } \\
\text { keiten }\end{array}$ & $\begin{array}{l}\text { R. Oblpp. einige feine } \\
\text { Rhonchi u. seltenes } \\
\text { Giemen; H Rauhig- } \\
\text { keiten } \\
\text { L. Spitze rauhes } \\
\text { vesiko.bronchial. } \\
\text { Atmen, Knacken }\end{array}$ \\
\hline 3 & $\begin{array}{l}\text { W.̈, } \\
18 \ddot{J}, \\
\text { Schüler }\end{array}$ & $\begin{array}{l}\text { Vetter und Cousine } \\
\text { an Tuberkulose ge- } \\
\text { storben. Vor } 1 / 2 \mathrm{~J} . \\
\text { Pleuritis sicca } \\
\text { dextra,späterHusten } \\
\text { und spärliche Blu- } \\
\text { tungen. Seit } 7 \text { Woch. } \\
\text { in Davos ohne Erfolg }\end{array}$ & - & $\begin{array}{l}\text { R. bis 3. Interkostal- } \\
\text { raum Mitte Scapulae } \\
\text { Schallkürzung, } \\
\text { hinten unten breite } \\
\text { Schwarte. Vorn bis } \\
\text { 3. Interkostalraum } \\
\text { Inspirium ver- } \\
\text { schärft, ebenso } \\
\text { hinten. In Fossa } \\
\text { supraspinata nach } \\
\text { Husten in der Tiefe } \\
\text { feine feuchte } \\
\text { Rhonchi. L hinten } \\
\text { Spitze Rhonchi an- } \\
\text { gedeutet }\end{array}$ & $\begin{array}{l}\text { L. Spitze hinten } \\
\text { rauhes Atmen, keine } \\
\text { Rhonchi, auch rechts } \\
\text { keine Rhonchi }\end{array}$ \\
\hline 4 & 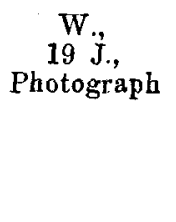 & $\begin{array}{l}\text { Vor } 1 \text { Jahr Pleuritis } \\
\text { und spitzenkatarrb. } \\
6 \text { Monate in Davos, } \\
\text { zu Hause Ruckfall }\end{array}$ & - & $\begin{array}{l}\text { L. Seite schleppt. } \\
\text { L. Spitze u. Lingula } \\
\text { vesiko-bronchial. } \\
\text { Atmen und Knattern }\end{array}$ & $\begin{array}{l}\text { L. Oberlappen } \\
\text { schleppt. Über Lin- } \\
\text { gula rauhes Atmen, } \\
\text { ab und zu ein grober } \\
\text { Bronchialrhonchus. } \\
\text { Hinten unten raubes } \\
\text { Atmen }\end{array}$ \\
\hline 5 & $\begin{array}{l}\text { St., } \\
46 \mathrm{~J}_{, 2} \\
\text { Schmied }\end{array}$ & $\begin{array}{c}\text { Seit } 1 \frac{1}{2} \text { Jahren } \\
\text { krank, aber stets } \\
\text { gearbeitet }\end{array}$ & - & $\begin{array}{l}\text { R. Spitze rel. Dpfg. } \\
\text { vesiko-bronchial. } \\
\text { Atmen mit Rauhig. } \\
\text { keiten. Atmung } \\
\text { überall leise. }\end{array}$ & $\begin{array}{l}\text { Über beiden Spitzen } \\
\text { reines vesiko.bron- } \\
\text { chial. Atmen } \\
\text { (Narbenatmen) }\end{array}$ \\
\hline 6 & $\begin{array}{l}\text { Z., } \\
48 \stackrel{\text { J., }}{\text { Beamter }}\end{array}$ & $\begin{array}{c}\text { Kränklich seit } \\
\text { Jahren. Vor 10 J. } \\
\text { Syphilis, seitdem } \\
\text { heiser. Lungenkrank } \\
\text { seit Wochen. Aus- } \\
\text { wurf. T.-B. zahlreich } \\
\text { nachgewiesen }\end{array}$ & - & $\begin{array}{l}\text { Über beiden Spitzen } \\
\text { vesiko-bronchial. } \\
\text { Atmen und Rhonchi. } \\
\text { Rauschen über bei- } \\
\text { den Lungen }\end{array}$ & $\begin{array}{l}\text { Über den Spitzen } \\
\text { vesiko-bronchial. } \\
\text { Atmen, Links vorn } \\
\text { oben einzelne Rhon- } \\
\text { chi. Kein Rauschen } \\
\text { mehr }\end{array}$ \\
\hline
\end{tabular}


Stadium 2.

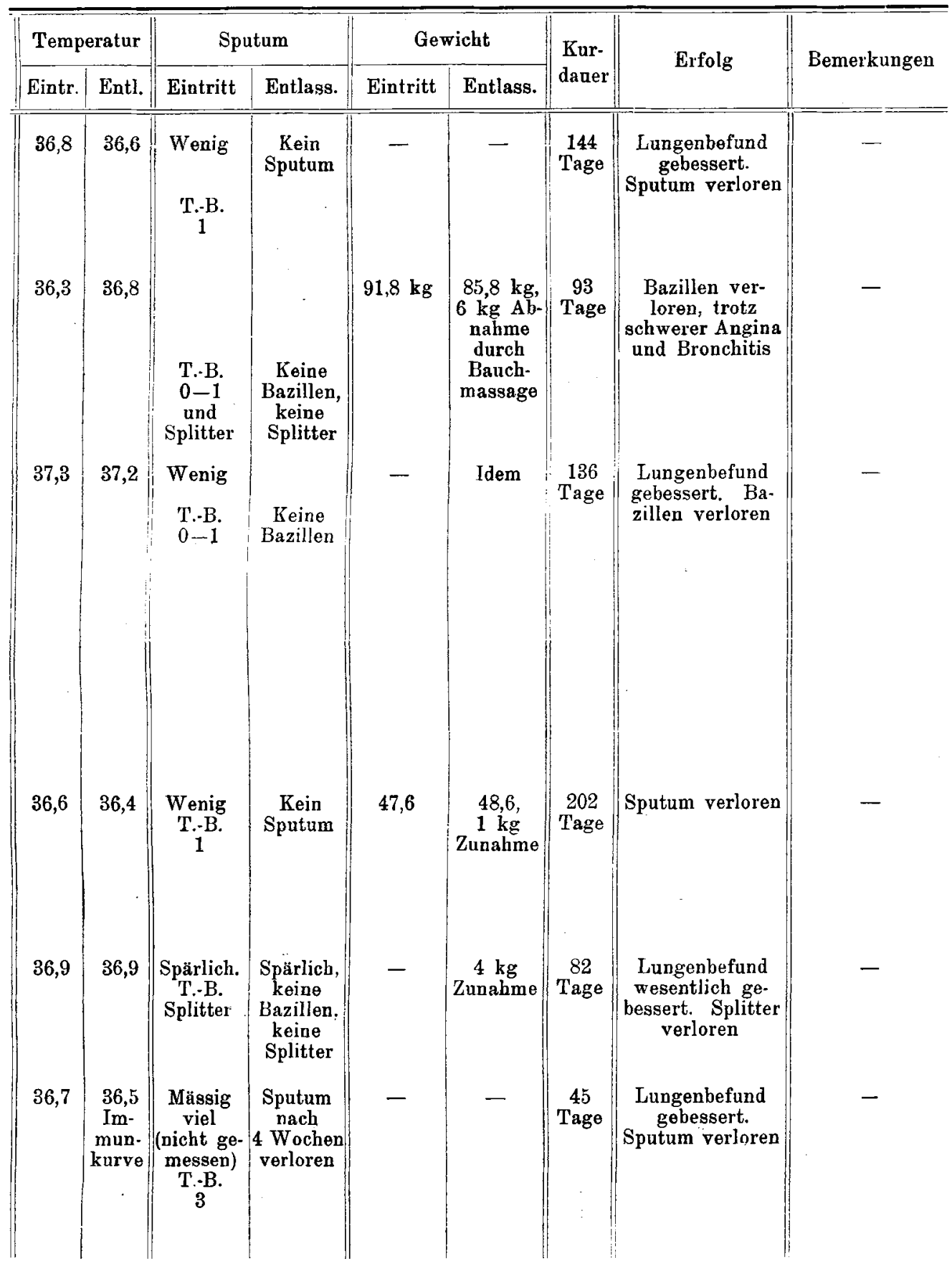




\begin{tabular}{|c|c|c|c|c|c|}
\hline \multirow{2}{*}{$\mathrm{Nr}$. } & \multirow{2}{*}{$\begin{array}{l}\text { Name, } \\
\text { Alter, } \\
\text { Beruf }\end{array}$} & \multirow{2}{*}{ Anamuese } & \multirow{2}{*}{$\begin{array}{c}\text { Kom- } \\
\text { plikationen }\end{array}$} & \multicolumn{2}{|c|}{ Lungenbefund } \\
\hline & & & & Eintritt & Entlassung \\
\hline 7 & $\begin{array}{l}\text { Z., } \\
25 \text { J., } \\
\text { Frau }\end{array}$ & $\begin{array}{l}\text { Krank seit } 2 \text { Jahren. } \\
\text { Frühere Kuren in } \\
\text { Davos und Leysin. } \\
\text { Auswurf wenig, aber } \\
\text { bazillenhaltig. Seit } \\
\text { dem 14. Jahr rezidi- } \\
\text { vierende Pleuritis } \\
\text { sicca }\end{array}$ & - & $\begin{array}{l}\text { R. Oberlappen relat. } \\
\text { Dämpfung, scharfes } \\
\text { vesiko-bronchial. } \\
\text { Atmen, in der Spitze } \\
\text { einige exspiratori- } \\
\text { sche feuchte } \\
\text { Rhonchi. Interska- } \\
\text { pular einige mittlere } \\
\text { Rhonchi. } \\
\text { L. Unterlappen, bes. } \\
\text { nach Basis żu, zahl. } \\
\text { reiche knatternde } \\
\text { Rhonchi }\end{array}$ & $\begin{array}{c}\text { Keine Rhonchi, } \\
\text { keine Rauhigkeiten }\end{array}$ \\
\hline 8 & $\begin{array}{l}\text { C., } \\
27 \mathrm{~J} ., \\
\text { Frau }\end{array}$ & $\begin{array}{c}\text { Seit 11/2 Jahr. Brust- } \\
\text { schmerzen. Vorbe- } \\
\text { handelt mit I.K. }\end{array}$ & - & $\begin{array}{l}\text { Hinten überall hoch- } \\
\text { gradig abgeschwäch- } \\
\text { tes und rauschendes } \\
\text { Atmen mit Aus- } \\
\text { nabme des l. Unter- } \\
\text { lappens }\end{array}$ & $\begin{array}{c}\text { Rauschen noch vor- } \\
\text { handen, aber ge- } \\
\text { ringer }\end{array}$ \\
\hline 9 & $\begin{array}{l}\text { S., } \\
22 \text { J., } \\
\text { Frau }\end{array}$ & \begin{tabular}{|l} 
Schwester an Tbc. $\dagger$. \\
Vor 12 Jahr. schwere \\
Meningitis, vor 8 \\
Jahren Lungenent- \\
ziindung, 2 Monate \\
dauernd; vor 3 Jahr. \\
Pleurit. sicca dextra, \\
die sich vor 2 Jahren \\
wiederholte, mit \\
Bronchopneumonie \\
verbunden. - Starke \\
Hämoptoe $(400$ ccm). \\
Seit 8 Monaten im \\
Davoser Sanatorium; \\
die letzen 2 Monate \\
Behandlung m. ATO. \\
Befinden nach \\
wie vor schlecht
\end{tabular} & - & $\begin{array}{l}\text { Brustumfang } 82 / 88 . \\
- \text { R. v. o. und l. h. } \\
\text { o. rel. Dämpfung. } \\
\text { Über beiden Oblpp. } \\
\text { rauhes Atmen. } \\
\text { R. giemendes Ge- } \\
\text { räusch über Oblpp. } \\
\text { und Mttlpp. zu hören. } \\
\text { R. h. interskapular } \\
\text { rauhes undrauschen- } \\
\text { des Atmen }\end{array}$ & $\begin{array}{l}\text { Brustumfang } 80 / 89 . \\
\text { R. Spitze abge- } \\
\text { schwächtes, etwas } \\
\text { rauschendes Atmen, } \\
\text { h. über der Spitze } \\
\text { Spur von Broncho- } \\
\text { phonie. - } \\
\text { Sonst uberall Vesi- } \\
\text { kuläratmen. Rau- } \\
\text { schen fast ver- } \\
\text { schwunden }\end{array}$ \\
\hline 10 & $\begin{array}{c}\text { C., } \\
23 \text { J., } \\
\text { Student }\end{array}$ & $\begin{array}{c}\text { Vor 1 Jahr Lungen- } \\
\text { katarrh konstatiert. } \\
\text { Husten, Schwäche, } \\
\text { Brustschmerzen, vor } \\
\text { 3 Wochen nach In- } \\
\text { fluenza Verschlim- } \\
\text { merung }\end{array}$ & Struma & $\begin{array}{l}\text { Über beiden Spitzen } \\
\text { Ravschen, rauhes } \\
\text { vesiko-bronch. Ex- } \\
\text { spirium. } \\
\text { L. Spitze hinten } \\
\text { feines Knacken. } \\
\text { Überall lautes rau- } \\
\text { schendes, zu vesiko- } \\
\text { bronchial. Charakter } \\
\text { neigendes Atmen }\end{array}$ & $\begin{array}{c}\text { Über den Spitzen } \\
\text { verschärftes reines } \\
\text { Vesikuläratmen. } \\
\text { Rauschen über den } \\
\text { Lungen noch vor- } \\
\text { handen }\end{array}$ \\
\hline 11 & $\begin{array}{c}\text { L., } \\
22 \mathrm{~J}_{.,} \\
\text {ökonom }\end{array}$ & $\begin{array}{l}\text { Vater an Tbe. } \dagger \\
\text { Ebenso Mutter und } \\
\text { Bruder. - Krank } \\
\text { seit 3 Monaten. - } \\
\text { Nachtschweisse ; } \\
\text { Appetit schlecht. }\end{array}$ & - & $\begin{array}{c}\text { Starkes Rauschen } \\
\text { uber beiden Lungen. } \\
\text { L. Untlpp. Inspi- } \\
\text { ration etwas vesiko- } \\
\text { bronchial mit inter- } \\
\text { poniertem Rauschen }\end{array}$ & $\begin{array}{l}\text { R. Spitze vesiko- } \\
\text { bronchial, rein; Spur } \\
\text { rel. Dpfg. - Sonst } \\
\text { überall vesikuläres } \\
\text { Atmen }\end{array}$ \\
\hline
\end{tabular}




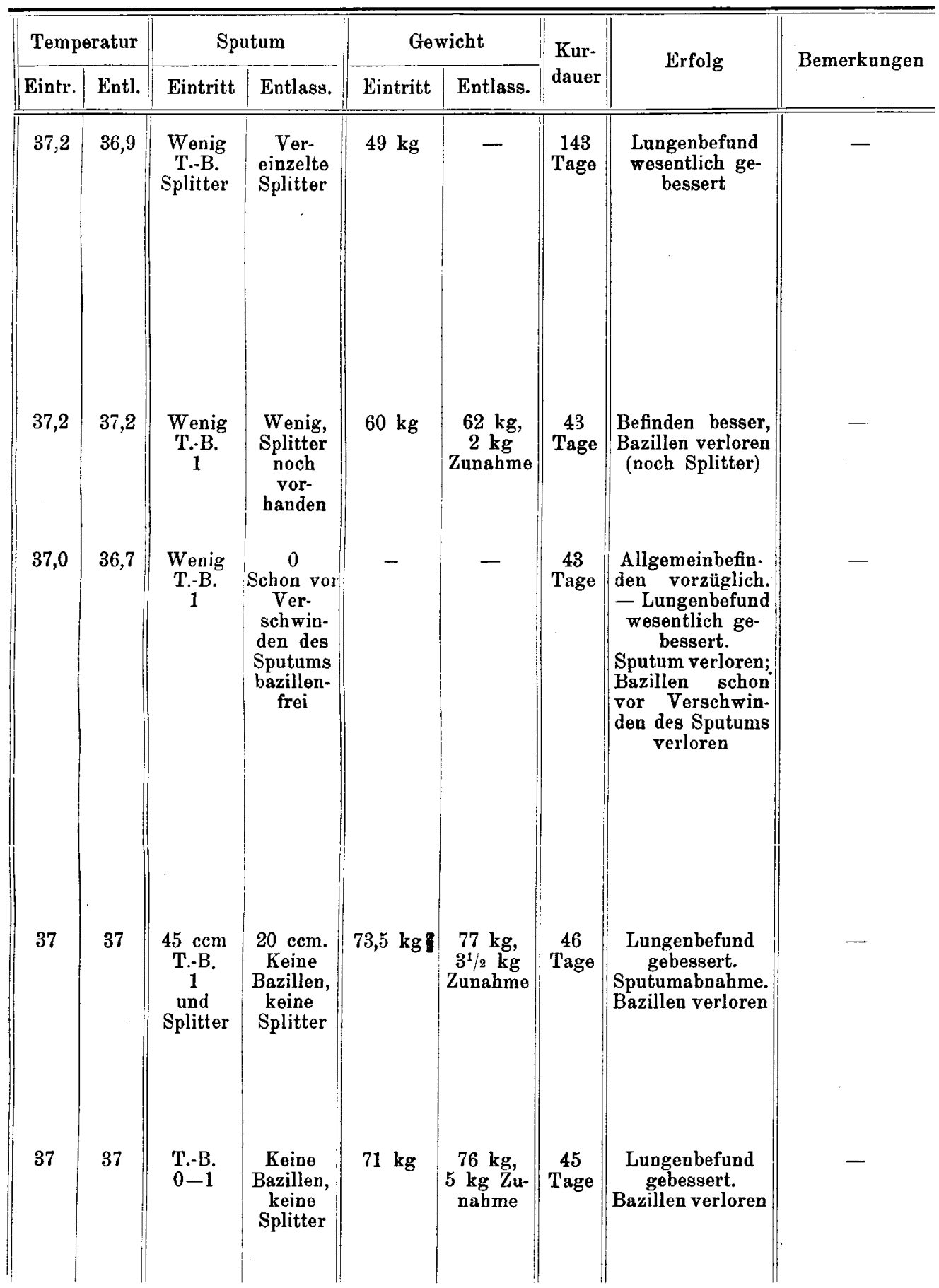




\begin{tabular}{|c|c|c|c|c|c|}
\hline \multirow{2}{*}{$\mathrm{Nr}$. } & \multirow{2}{*}{$\begin{array}{l}\text { Name, } \\
\text { Alter, } \\
\text { Beruf }\end{array}$} & \multirow{2}{*}{ Anamnese } & \multirow{2}{*}{$\begin{array}{c}\text { Kom- } \\
\text { plikationen }\end{array}$} & \multicolumn{2}{|c|}{ Lungenbefund } \\
\hline & & & & Eintritt & Entlassung \\
\hline 12 & $\begin{array}{c}\text { B., } \\
20 \mathrm{~J} . \\
\text { Kaufmann }\end{array}$ & $\begin{array}{l}\text { Hatte als Kind Hals- } \\
\text { drüsen- und Hals- } \\
\text { wirbeltuberkulose.- } \\
\text { Drüsen operativ be- } \\
\text { handelt. - T.-B. im } \\
\text { Auswurf nachge- } \\
\text { wiesen }\end{array}$ & - & $\begin{array}{l}\text { Über r. Oblpp. u. } \\
\text { Mttlpp. vesiko-bron- } \\
\text { chial. Atmen; über } \\
\text { Mttlpp. vereinzelte } \\
\text { Rhonchi }\end{array}$ & $\begin{array}{l}\text { Uber d. r. Spitze et- } \\
\text { was rauhes Atmen; } \\
\text { sonst nichts. - } \\
\text { Rhonchi verschwun- } \\
\text { den }\end{array}$ \\
\hline 13 & $\begin{array}{l}\text { P., } \\
23 \text { J., } \\
\text { Fräulein }\end{array}$ & $\begin{array}{l}\text { Krank seit }{ }^{3} \text {; Jahren. } \\
\text { In Görbersdorf 3 Mo- } \\
\text { nate lang (bis jetzt); } \\
\text { daselbst mit ATO } \\
\text { behandelt. - Husten } \\
\text { und Auswurf; kein } \\
\text { Appetit }\end{array}$ & - & $\begin{array}{l}\text { R. Spitze vesiko- } \\
\text { broncho - bronchial. } \\
\text { Atmen, H. rauh. } \\
\text { R. H. U. und seitlich } \\
\text { abgeschwächtes und } \\
\text { nicht ganz reines } \\
\text { Atmen (früher Pleu- } \\
\text { ritis) }\end{array}$ & $\begin{array}{l}\text { R. Spitze Narben- } \\
\text { atmen, keine } \\
\text { Rhonchi }\end{array}$ \\
\hline 14 & $\begin{array}{l}\text { St., } \\
43 \text { J., } \\
\text { Architekt }\end{array}$ & $\begin{array}{l}\text { Vater an Tbc. ge- } \\
\text { storben. Husten seit } \\
7 \text { Monaten. Auswurf } \\
\text { nicht untersucht }\end{array}$ & - & $\begin{array}{l}\text { R. hinten stark ab- } \\
\text { geschwächtes und } \\
\text { rauschendes Atmen. } \\
\text { L. hinten ebenfalls } \\
\text { Rauschen, Atemge- } \\
\text { räusch weniger ab- } \\
\text { geschwächt wie R. }\end{array}$ & $\begin{array}{l}\text { R. Sp. vorn ver- } \\
\text { scliärftes reinesVesi- } \\
\text { kulär-Atmen, infra- } \\
\text { klavikular Spur von } \\
\text { Rauschen. Ende des } \\
\text { Inspiriumsvesikulär. } \\
\text { Atmen } \\
\text { R. hinten Oberl. vesi- } \\
\text { kulär. Atmen mit et- } \\
\text { was Rauschen; } \\
\text { Unterl. ebenso, aber } \\
\text { sehrwenigRauschen. } \\
\text { L. hinten über Ober- } \\
\text { undUnterlappen voll- } \\
\text { kommen reines } \\
\text { scharfes vesikulär. } \\
\text { Atmen }\end{array}$ \\
\hline 15 & $\begin{array}{l}\text { M., } \\
26 \text { J., } \\
\text { Techniker }\end{array}$ & $\begin{array}{l}\text { Krank seit } 4 \text { Jahren. } \\
\text { Ausworf und Nacht- } \\
\text { schweisse }\end{array}$ & - & $\begin{array}{l}\text { Utber beiden Spitzen } \\
\text { rel. Dpfg. und uber } \\
\text { den Oblpp. leises } \\
\text { vesiko-bronchial. } \\
\text { Atmen und } \\
\text { Rauschen } \\
\text { R. Untlpp. an der } \\
\text { Basis Knattern, } \\
\text { vesiko-bronchial. } \\
\text { Atmen }\end{array}$ & $\begin{array}{l}\text { R. Oblpp. vesiko- } \\
\text { bronchial; nirgends } \\
\text { Rhonchi }\end{array}$ \\
\hline 16 & $\begin{array}{l}\text { R., } \\
41 \stackrel{\text { J., }}{\text { Arzt }}\end{array}$ & $\begin{array}{c}\text { Seit 13 Jahren krank. } \\
\text { T.-B. nachgewiesen. } \\
\text { - Syphilis }\end{array}$ & - & $\begin{array}{l}\text { L. Oblpp. Knattern } \\
\text { vorn - Vl. Rippe; } \\
\text { H. verschärftes } \\
\text { Atmen } \\
\text { R. Mttlpp. vereinzel- } \\
\text { tes Knattern }\end{array}$ & $\begin{array}{l}\text { L. Oblpp. keine } \\
\text { Rhonchi mehr; Er- } \\
\text { öffnungsknattern }\end{array}$ \\
\hline
\end{tabular}




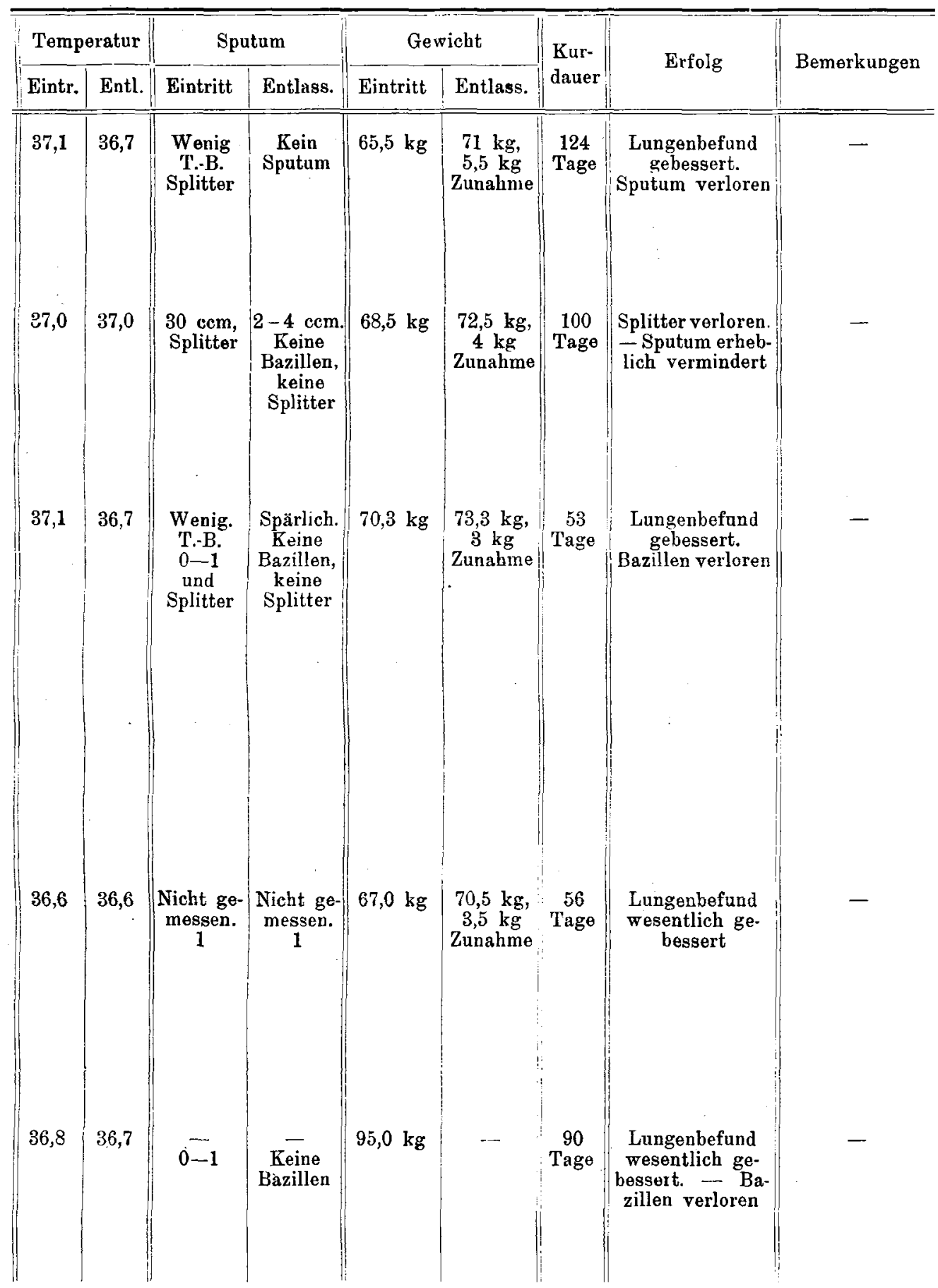




\begin{tabular}{|c|c|c|c|c|c|}
\hline \multirow{2}{*}{ Nr. } & \multirow{2}{*}{$\begin{array}{l}\text { Name, } \\
\text { Alter, } \\
\text { Beruf }\end{array}$} & \multirow{2}{*}{ Anamnese } & \multirow{2}{*}{$\begin{array}{c}\text { Kom- } \\
\text { plikationen }\end{array}$} & \multicolumn{2}{|c|}{ Lungenbefund } \\
\hline & & & & Eintritt & Entlassung \\
\hline 17 & $\begin{array}{l}\text { L., } \\
45 \mathrm{~J} ., \\
\text { Frau }\end{array}$ & $\begin{array}{l}\text { Gatte an Tbc. t. - } \\
\text { Tochter tuberkulös. } \\
\text { In letzter Zeit } \\
\text { Schmerzen in der l. } \\
\text { Brustseite. Mor- } \\
\text { gens Husten und } \\
\text { Auswurf }\end{array}$ & - & $\begin{array}{l}\text { R. Spitze rel. Dpfg., } \\
\text { vesiko-bronchial. } \\
\text { Atmen; Exspirium } \\
\text { bronchial ; keine } \\
\text { Rhonchi (Narben). } \\
\text { L. h. u. vesiko-bron- } \\
\text { chial. Atmen und } \\
\text { einige krepitierende } \\
\text { Rhonchi }\end{array}$ & $\begin{array}{l}\text { R. vorn ves.br. Ex. } \\
\text { spir. verlängert, ab- } \\
\text { wärts scharf vesik., } \\
\text { hinten Sp. wie vorn, } \\
\text { interskap. ves. - br. } \\
\text { ohne Rauhigkeiten. } \\
\text { Unterl. scharf vesi- } \\
\text { kulär } \\
\text { L. vorn Sp. ves.-br., } \\
\text { rein, infrakl. scharf } \\
\text { vesik. } \\
\text { Hinten Sp. leise ves.- } \\
\text { br. Unterl. ves.br., } \\
\text { an der Basis etwas } \\
\text { Knacken }\end{array}$ \\
\hline 18 & $\begin{array}{l}\mathrm{R} . \\
21 \mathrm{~J} ., \\
\text { Fräulein }\end{array}$ & $\begin{array}{l}\text { Vater an Tbc. ge- } \\
\text { storben. Vor 5Jahren } \\
\text { Lungenblutung. Mor- } \\
\text { gens etwas Husten } \\
\text { und Auswurf. Vor } \\
\text { 3 Jahren rechts- } \\
\text { seitige Pleuritis }\end{array}$ & - & $\begin{array}{l}\text { R.Oberl.relat.Dämp- } \\
\text { fung. Ưber derganzen } \\
\text { r. Lunge abge- } \\
\text { schwächtes rauhes } \\
\text { und rauschendes At- } \\
\text { men, dabei hinten } \\
\text { fast überall feines } \\
\text { Knarren }\end{array}$ & $\begin{array}{l}\text { R. Spitze vesiko- } \\
\text { bronchial.Atmen und } \\
\text { Knattern. UUber dem } \\
\text { r.Oberl.noch Raubig- } \\
\text { keiten. } \\
\text { L. Spitzevesiko-bron- } \\
\text { chal, rauh und etwas } \\
\text { Knattern }\end{array}$ \\
\hline 19 & $\begin{array}{c}\text { O., } \\
38 \text { J., } \\
\text { Kaufmann }\end{array}$ & $\begin{array}{l}\text { Ein Bruder lungen- } \\
\text { krank. Seit12 Jahren } \\
\text { Lungenspitzen- } \\
\text { katarrh; Tuberkel- } \\
\text { bazillen nachge- } \\
\text { wiesen. Vor11Jahren } \\
2 \text { mal Hämoptoe. - } \\
\text { Klagen über Husten }\end{array}$ & - & $\begin{array}{l}\text { L. Oblpp. vesiko- } \\
\text { bronchial.Atmen, Al- } \\
\text { veolar-Knattern u. } \\
\text { Knacken. - R.Oblpp. } \\
\text { u. Mttlpp. stark ab- } \\
\text { geschw. rauhes At- } \\
\text { men, vesiko-bron- } \\
\text { chial, Knacken }\end{array}$ & $\begin{array}{l}\text { L. Oblpp. vesiko- } \\
\text { bronchial.Atmen,Al- } \\
\text { veolar-Knattern. } \\
\text { R. Oblpp. u. Mttlpp. } \\
\text { stark abgeschw., } \\
\text { rauhes Atmen }\end{array}$ \\
\hline 20 & $\begin{array}{l}\text { K.,., } \\
32 \text { J., } \\
\text { Journalist }\end{array}$ & $\begin{array}{l}\text { Seit } 7 \text { Jahren krank; } \\
\text { vor } 4 \text { Jabren leichte } \\
\text { Hämoptoe, danach } \\
\text { Davos } 6 \text { Monate ohne } \\
\text { Erfolg. - Vor } \\
\text { 3Jahren wieder } 5 \text { Mo. } \\
\text { nate in Davos, seit } \\
\text { 1 Jahr ununter- } \\
\text { brochen in Davos, } \\
\text { fühlt sich trotzdem } \\
\text { immer schlechter. } \\
\text { Sputum } 20 \text { - 30 ccm; } \\
\text { Tb. nachgewiesen }\end{array}$ & - & $\begin{array}{l}\text { R. Oblpp. vesiko- } \\
\text { bronchiale Atmung } \\
\text { und Knattern, bes. } \\
\text { hinten. } \\
\text { L. Spitze vorn vesiko- } \\
\text { bronchial. Atmen }\end{array}$ & $\begin{array}{l}\text { R. Oblpp. Verdacht } \\
\text { auf Halb-Rhonchi } \\
\text { nach Husten } \\
\text { L. Spitze rauh }\end{array}$ \\
\hline 21 & $\begin{array}{l}\text { H., } \\
27 \text { J., } \\
\text { Beamter }\end{array}$ & $\begin{array}{l}\text { Seit 4Wochen krank, } \\
\text { Beginn mitHusten.- } \\
\text { Wenig Nacht- } \\
\text { schweisse, Schlaf } \\
\text { schlecht. - Tb. } \\
\text { nachgewiesen }\end{array}$ & - & $\begin{array}{l}\text { Spitzen rel. Dpfg., } \\
\text { rauhes Atmen; viel } \\
\text { Rauschen }\end{array}$ & $\begin{array}{l}\text { Spitzen rel. Dpfg., } \\
\text { reines vesiko-bron- } \\
\text { chial. Atmen. Kein } \\
\text { Rauschen }\end{array}$ \\
\hline
\end{tabular}




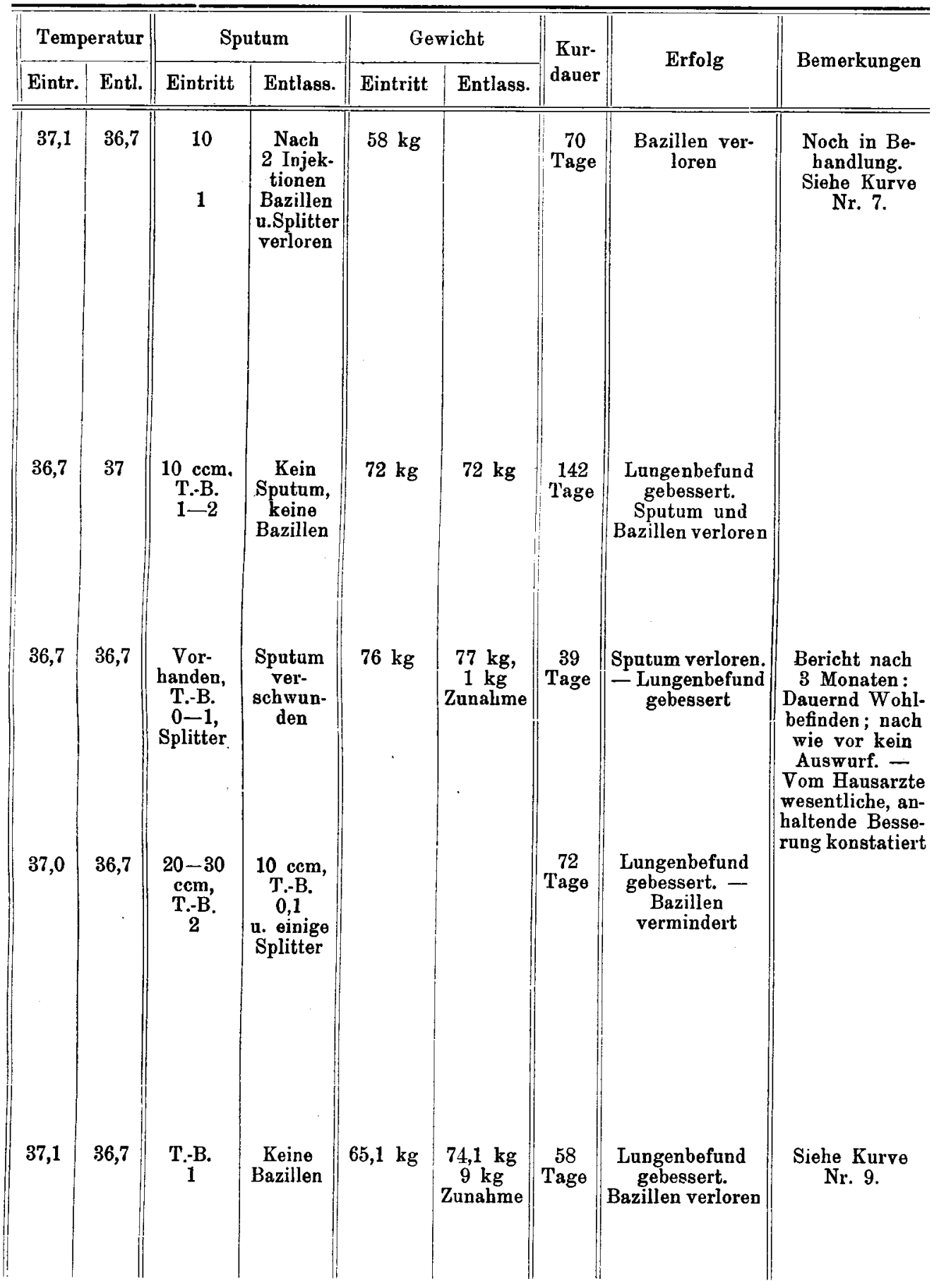




\begin{tabular}{|c|c|c|c|c|c|}
\hline \multirow{2}{*}{ Nr. } & \multirow{2}{*}{$\begin{array}{l}\text { Name, } \\
\text { Alter, } \\
\text { Beruf }\end{array}$} & \multirow{2}{*}{ Anamnese } & \multirow{2}{*}{$\begin{array}{c}\text { Kom- } \\
\text { plikationen }\end{array}$} & \multicolumn{2}{|c|}{ Lungenbefund } \\
\hline & & & & Eintritt & Entlassung \\
\hline 22 & $\begin{array}{l}\text { H., } \\
46 . \text { J., } \\
\text { Frau }\end{array}$ & $\begin{array}{l}\text { Vater war lungen- } \\
\text { krank. Vor 3 Jahren } \\
\text { Arosa, Besserung. - } \\
\text { Seit 3 Monaten wie- } \\
\text { der Husten und Aus- } \\
\text { wurf. Allgemeinbe- } \\
\text { finden schlecht. } \\
\text { Appetit mässig }\end{array}$ & - & $\begin{array}{l}\text { Über beiden Spitzen } \\
\text { rel. Dpfg., Vesico- } \\
\text { bronchial-Atmen und } \\
\text { hinten einzelne } \\
\text { Rhonchi }\end{array}$ & $\begin{array}{l}\text { Geringe rel. Dpfg. } \\
\text { über beiden Spitzen } \\
\text { u. Vesico-bronchial- } \\
\text { Atmen ohne Rhonchi }\end{array}$ \\
\hline 23 & $\begin{array}{l}\text { F. } \\
\text { Student }\end{array}$ & $\begin{array}{l}\text { Krank seit über 3Jahren. } \\
\text { Beginn mit Katarrh, Tb. } \\
\text { nachgewiesen. - Mehr- } \\
\text { fach klimatisehe Kuren; } \\
\text { wiederholt mit Tuber- } \\
\text { kulin behandelt; zuletzt } \\
\text { vor einem Jahr }\end{array}$ & - & $\begin{array}{c}\text { I. h. uber der Spitze } \\
\text { unreinesAtmen; iuber } \\
\text { beiden Oblpp. Vesico- } \\
\text { bronchial-Atmen }\end{array}$ & $\begin{array}{l}\text { Über den Spitzen } \\
\text { Narbenatmen, } \\
\text { vesico-bronchial, be- } \\
\text { sonders stark l. h. }\end{array}$ \\
\hline 24 & $\begin{array}{l}\text { B., } \\
\text { 39.,., } \\
\text { Sekretär }\end{array}$ & $\begin{array}{c}\text { Vor 2 Monaten akute } \\
\text { Erkältung }\end{array}$ & - & $\begin{array}{l}\text { R. Sp. Ves.-bronch., } \\
\text { einzelne Rhonchi. } \\
\text { Über beiden Lungen } \\
\text { Rauschen, besonders } \\
\text { stark über d. Oblpp. }\end{array}$ & $\begin{array}{l}\text { R. Spitze Narben- } \\
\text { atmen (vesico-bron- } \\
\text { chial); Rauschen } \\
\text { noch vorhanden }\end{array}$ \\
\hline 25 & $\begin{array}{c}\text { B., } \\
24 \text { J.; } \\
\text { Techniker }\end{array}$ & \begin{tabular}{|l}
10 Wochen krank \\
Beginn mit Pleuritis \\
sicca. - Darnach \\
mehrfacheBlutungen \\
10 kg Abnahme im \\
letzten Jahr
\end{tabular} & - & $\begin{array}{l}\text { R Spitze rel. Dpfg.; } \\
\text { Vesico-bronch.-At- } \\
\text { men über den Oblpp. } \\
\text { L. Spitze Vesico- } \\
\text { bronchial-Atmen } \\
\text { Rauscher }\end{array}$ & \begin{tabular}{|} 
R. Spitze rel. Dpfg., \\
Vesico-bronchial- \\
Atmen. \\
L. Spitze Vesico- \\
bronchial-Atmen; \\
interskapular rasche \\
Atmung, ab und zu \\
Knacken
\end{tabular} \\
\hline 26 & $\begin{array}{l}\text { N., } \\
55 \text { J., } \\
\text { Lehrer }\end{array}$ & $\begin{array}{l}\text { DiagnostischeTuber- } \\
\text { kulin-Injektionen (zu } \\
\text { Hause) ergaben Fie- } \\
\text { berreaktion }-39^{0} .- \\
\text { Morgens Sputum; } \\
\text { Tb. nicht gefunden }\end{array}$ & - & $\begin{array}{l}\text { R. Oblpp. schleppt; } \\
\text { abgeschwächtes un- } \\
\text { bestimmtes Atmen; } \\
\text { h. uber der Spitze } \\
\text { einzelnes Knacken. } \\
\text { L. Spitze ebenfalls } \\
\text { Knacken. Rauschen } \\
\text { über beiden Lungen }\end{array}$ & $\begin{array}{l}\text { Beide Spitzen vesico- } \\
\text { bronchial, keine } \\
\text { Rhonchi }\end{array}$ \\
\hline 27 & $\begin{array}{l}\text { L., } \\
\text { 33 J., } \\
\text { Beamter }\end{array}$ & 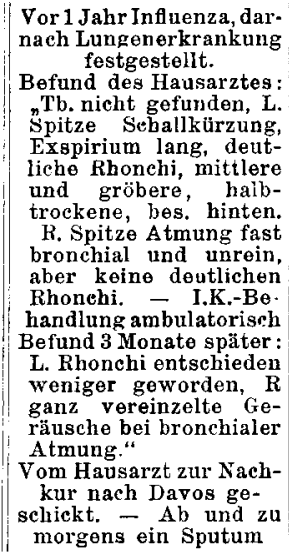 & 一 & $\begin{array}{l}\text { R. Oblpp. reines } \\
\text { Vesico-bronchial- } \\
\text { Atmen (scheint ge- } \\
\text { heilt). } \\
\text { L. Oblpp. vesico- } \\
\text { bronchial, vielleicht } \\
\text { etwas rauh (aber } \\
\text { nicht sicher) }\end{array}$ & $\begin{array}{l}\text { Eröffnungsknattern } \\
\text { über allen erkrank- } \\
\text { ten Partien. - Über } \\
\text { beiden Oblpp. reines } \\
\text { Vesico-bronchial- } \\
\text { Atmen }\end{array}$ \\
\hline
\end{tabular}




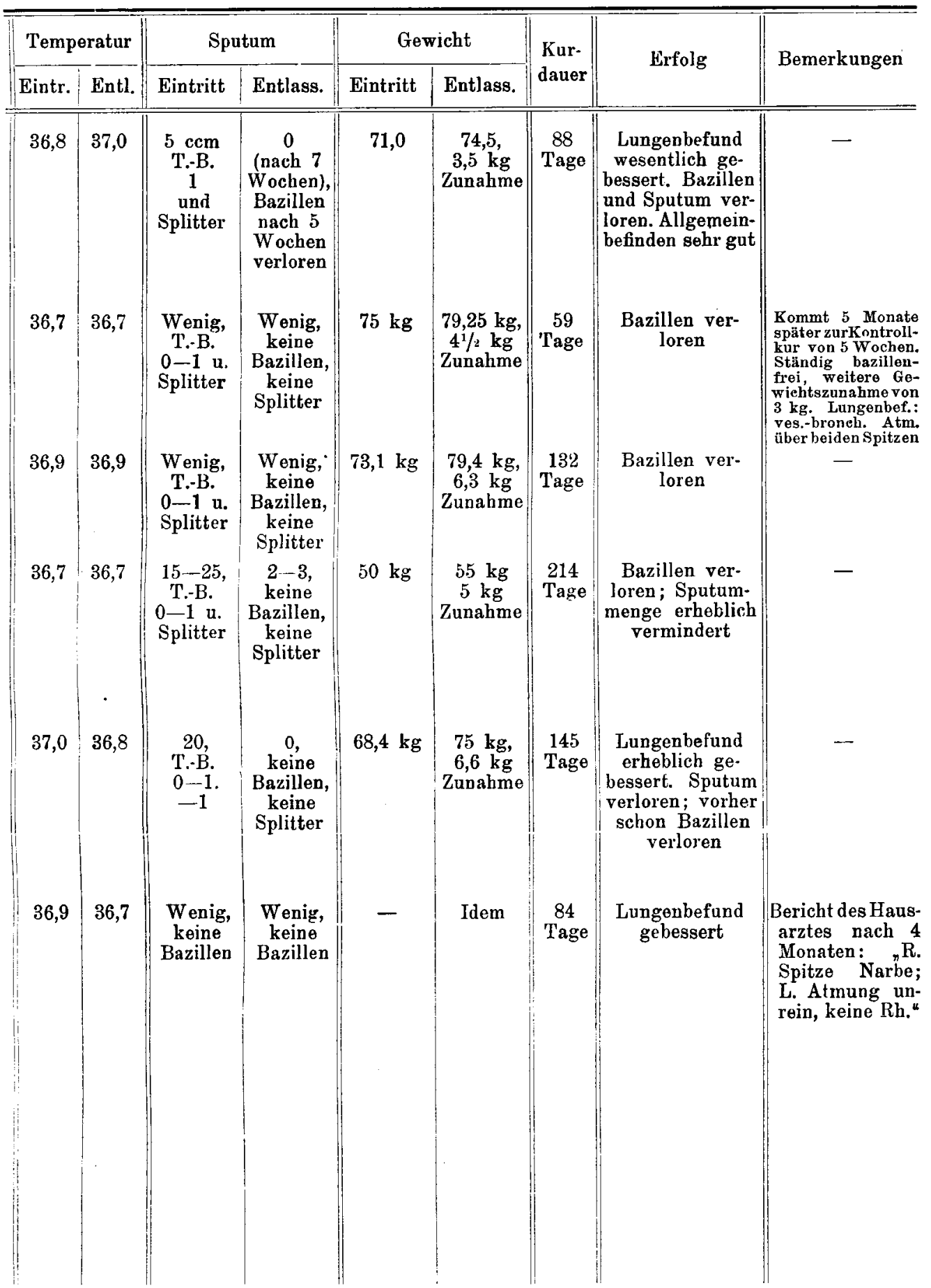




\begin{tabular}{|c|c|c|c|c|c|}
\hline \multirow{2}{*}{ Nr. } & \multirow{2}{*}{$\begin{array}{l}\text { Name, } \\
\text { Alter, } \\
\text { Beruf }\end{array}$} & \multirow{2}{*}{ Anamnese } & \multirow{2}{*}{$\begin{array}{c}\text { Kom- } \\
\text { plikationen }\end{array}$} & \multicolumn{2}{|c|}{ Lungenbefund } \\
\hline & & & & Eintritt & Entlassung \\
\hline 28 & $\begin{array}{l}\mathrm{R} . \\
17 \mathrm{~J} . \\
\text { Gymnasiast }\end{array}$ & $\begin{array}{l}\text { Vor } 5 \text { Monaten Plea- } \\
\text { ritis exsudativa sin., } \\
\text { nachher } 10 \mathrm{~kg} \text { Ge- } \\
\text { wichtsabnahme, in } \\
\text { letzter Zeit } 3 \mathrm{~kg} \text { Zu- } \\
\text { nahme, Mattigkeit. } \\
\text { Schwäche }\end{array}$ & $\begin{array}{c}\text { Starke } \\
\text { Anämie. }\end{array}$ & $\begin{array}{l}\text { L. Brustseite } \\
\text { schleppt stark. Oberl. } \\
\text { relative Dpfg.vesiko- } \\
\text { bronch. Atmen, ver- } \\
\text { cinzelte Rhonchi. } \\
\text { Broncholoquie über } \\
\text { der Spitze. Von } \\
\text { 4. Rippe abwärts ab- } \\
\text { solute Dpfg., Stimm- } \\
\text { fremitus und Atem- } \\
\text { geräusch stark ab- } \\
\text { geschwächt } \\
\text { (Schwarte). R. Spitze } \\
\text { Schallkürzung, ver- } \\
\text { längertes Exspirium, } \\
\text { vesiko-bronch. } \\
\text { Atmen, Rauschen } \\
\text { über beiden Lungen }\end{array}$ & $\begin{array}{l}\text { L. Oberlpp. relative } \\
\text { Dpfg., ves. - bronch. } \\
\text { Atmen, keine } \\
\text { Rhonchi. Von 4. R. } \\
\text { abwärts Schwarte } \\
\text { wie am Anfang. } \\
\text { R. Sp. Exspirium ver- } \\
\text { längert. } \\
\text { Kein Rauschen }\end{array}$ \\
\hline 29 & $\begin{array}{l}\text { v. B., } \\
29 \text { J., } \\
\text { Leamter }\end{array}$ & $\begin{array}{l}\text { Zweimal Lungenent- } \\
\text { zündung. Vor } \\
2 \text { Jahren Rippenfell. } \\
\text { entzündung rechts. } \\
\text { Halsdrüsen seit } \\
\text { 10 Jahren, jeder Be- } \\
\text { handlung trotzend, } \\
\text { nach Inzision blieb } \\
\text { eine Fistel zurück, } \\
\text { die nicht heilte }\end{array}$ & $\begin{array}{l}\text { Am Hals bds. } \\
\text { grosseD ü sen- } \\
\text { pake te, be- } \\
\text { stehend ausDrü- } \\
\text { sen von Hasel- } \\
\text { nuss- bis Tan- } \\
\text { beneigrösse. An } \\
\text { der l. Seite eine } \\
\text { Fistel mit } 6 \mathrm{~cm} \\
\text { langem Gang, } \\
\text { ans der sich } \\
\text { einige Tropfen } \\
\text { dieken Eiters } \\
\text { ausdricken } \\
\text { lassen. Im Eiter } \\
\text { werden Tb.-Ba- } \\
\text { zillen naehge- } \\
\text { wiesen. Hals- } \\
\text { umfang über der } \\
\text { Cartilago thy- } \\
\text { reoidea } 42 \mathrm{~cm} \text {, } \\
\text { unterhalb der- } \\
\text { selben } 40 \mathrm{~cm}\end{array}$ & $\begin{array}{l}\text { Beide Spitzen rela- } \\
\text { tive Dämpfung, } \\
\text { rechtsmebrwielinks. } \\
\text { R.O.L. unbestimm- } \\
\text { tes Atmen, Broncho- } \\
\text { loquie, hinten kni- } \\
\text { sternde Rhonchi. } \\
\text { Unter dem Angulus } \\
\text { scapulae Reiben. } \\
\text { Rauschen über Un- } \\
\text { ter- u. Mittellappen, } \\
\text { über letzterem ves.- } \\
\text { bronch. Atmung. } \\
\text { L. Spitze scharfe At- } \\
\text { mung, verlängertes } \\
\text { Exspirium, v. und h. } \\
\text { überall Rauschen }\end{array}$ & $\begin{array}{l}\text { Über beiden Spitzen } \\
\text { relative Dämpfung, } \\
\text { ves.bronch. Atmen, } \\
\text { über der R. Spitze } \\
\text { Broncholoquie. } \\
\text { Kein Rauschen }\end{array}$ \\
\hline 30 & $\begin{array}{l}\text { L., } \\
29 \text { J., } \\
\text { Vikar }\end{array}$ & $\begin{array}{l}\text { Seit } 1 \text { Jahr Husten } \\
\text { und Schmerzen auf } \\
\text { der r. Brustseite. Vor } \\
2 \text { Monaten Bronchitis } \\
\text { mit Fieber, zurzeit } \\
\text { kein Fieber }\end{array}$ & - & $\begin{array}{l}\text { Über beiden Spitzen reia. } \\
\text { tive Dämpfung, R. mehr } \\
\text { als L. R. Spitze bezw. } \\
\text { Oberlappen ves.-bronch. } \\
\text { rauh, vereinzelt in der } \\
\text { Spitze Knacken, hinten } \\
\text { R. Spitze Kavernoloquie } \\
\text { L. Spitze schwach } \\
\text { bronchial. Überall } \\
\text { starkes Rausehen }\end{array}$ & $\begin{array}{c}\text { R. Lunge abge- } \\
\text { schwächtes Atmen. } \\
\text { Rhonchi nirgends zu } \\
\text { hören }\end{array}$ \\
\hline 31 & $\begin{array}{l}\text { E.. } \\
\text { Frau }\end{array}$ & $\begin{array}{c}\text { Seit } 8 \text { Monaten } \\
\text { krank. Husten und } \\
\text { Auswurf. }\end{array}$ & Nourasthenie & $\begin{array}{l}\text { R. Oberlappen und } \\
\text { L. Spitze relative } \\
\text { Dämpfung. Über r. } \\
\text { Oberlappen Atmen } \\
\text { vesiko-bronchial, } \\
\text { raub. In der Spitze } \\
\text { Knacken. Broncho- } \\
\text { loquie. L. Spitze } \\
\text { idem }\end{array}$ & $\begin{array}{l}\text { Beide Spitzen rela- } \\
\text { tive Dämpfung und } \\
\text { vesiko-bronch. Atm. } \\
\text { mit Spur Broncho- } \\
\text { loquie. KeineRbonchi }\end{array}$ \\
\hline
\end{tabular}




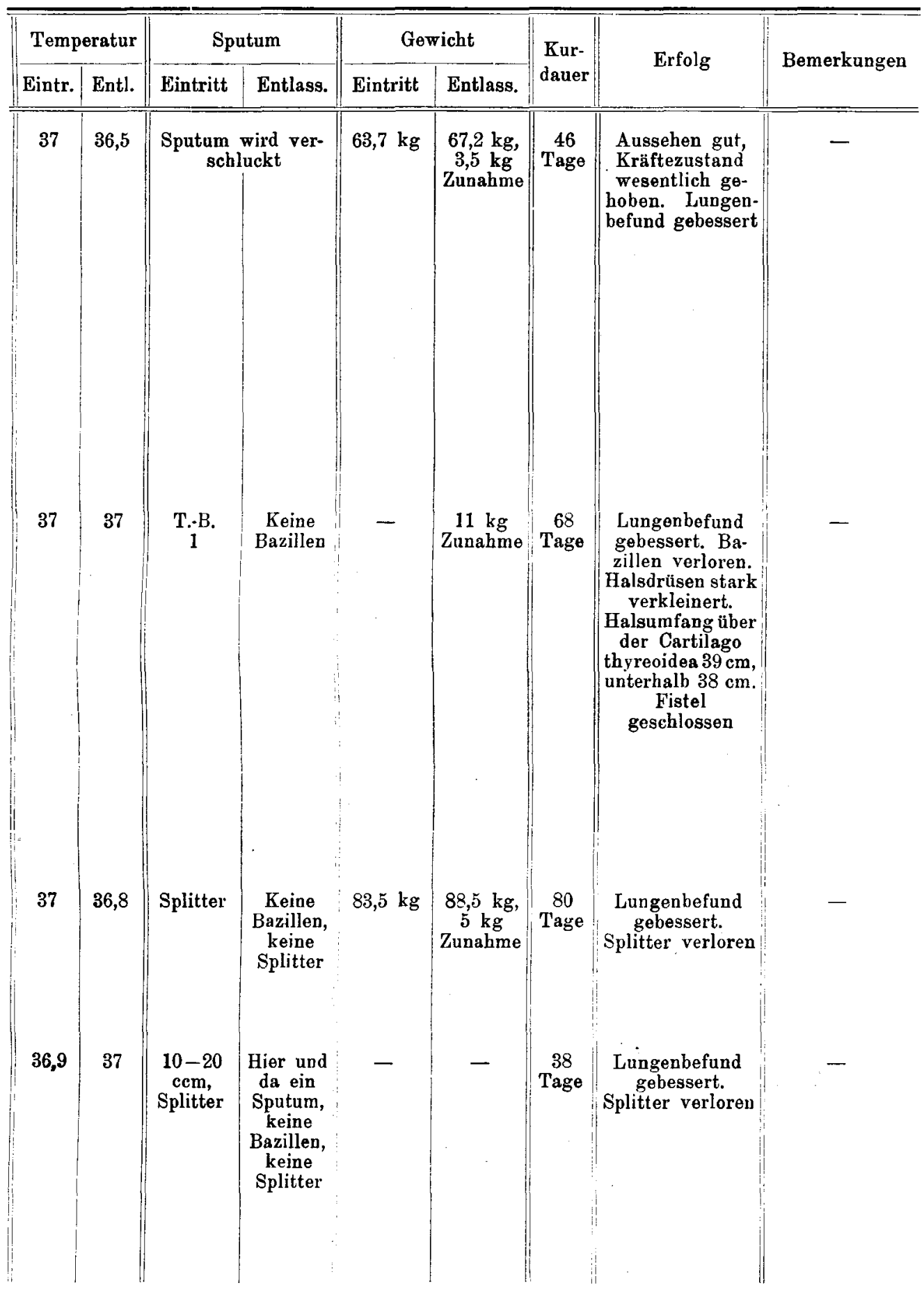




\begin{tabular}{|c|c|c|c|c|c|}
\hline \multirow{2}{*}{ Nr. } & \multirow{2}{*}{$\begin{array}{l}\text { Name, } \\
\text { Alter, } \\
\text { Beruf }\end{array}$} & \multirow{2}{*}{ Anamnese } & \multirow{2}{*}{$\begin{array}{c}\text { Kom- } \\
\text { plikationen }\end{array}$} & \multicolumn{2}{|c|}{ Lungenbefund } \\
\hline & & & & Eintritt & Entlassung \\
\hline 32 & $\begin{array}{l}\text { G., } \\
32 \text { J., } \\
\text { Frau }\end{array}$ & $\begin{array}{l}1 \text { Bruder an Tb. } \nmid .- \\
\text { Vor } 6 \text { Monaten } \\
\text { rechtsseitigeLungen- } \\
\text { entzündung, danach } \\
\text { Rippenfellreizung, } \\
\text { und bald darauf } \\
\text { wurde Erkrankung } \\
\text { beider Spitzen fest- } \\
\text { gestellt. - } \\
\text { Vor } 2 \text { Monaten mit } \\
\text { Rücksicht auf die } \\
\text { Krankheit: künst- } \\
\text { licher Abort im } \\
\text { III. Monat }\end{array}$ & - & $\begin{array}{l}\text { Über beid. Spitzen } \\
\text { rel. Dämpf.; LV. bis } \\
\text { II. Rippe, H. bis } \\
\text { Mitte Scap. feines } \\
\text { Knisterrasseln. } \\
\text { RV. bis IlI. Rippe, } \\
\text { H. gegen Angulus } \\
\text { sich verlierend, } \\
\text { gleichfalls knist. } \\
\text { Rhonchi, dieV.etwas } \\
\text { gröber sind. } \\
\text { Rauschen mehrfach, } \\
\text { bes. RHU. }\end{array}$ & $\begin{array}{l}\text { L. O.-Lappen relat. } \\
\text { Dämpf., Alveolar- } \\
\text { knattern V. und H., } \\
\text { die ganze inspirat. } \\
\text { Phase vom ersten } \\
\text { Drittel ab begleitend. } \\
\text { R. Spitze wie } \mathrm{I} \text {. }\end{array}$ \\
\hline 33 & $\begin{array}{l}\text { G., } \\
34 \text { J., } \\
\text { Frrl. }\end{array}$ & $\begin{array}{l}\text { Vor } 19 \text { Jahren länger } \\
\text { dauernder Husten. } \\
\text { Seit zwei Jahren be- } \\
\text { steht wieder Husten; } \\
\text { zeitw eise etwas Blut } \\
\text { gehustet. - Durch } \\
\text { Tierversuch Tb. fest- } \\
\text { gestellt }\end{array}$ & - & $\begin{array}{l}\text { R. Spitze stärker } \\
\text { ged. als L. - R. } \\
\text { Spitze u. U.-Lappen } \\
\text { ves.-bronch. Knack. } \\
\text { - L. Spitze H. rauh. } \\
\text { Atmen; intraskap. } \\
\text { einige Rhonchi }\end{array}$ & $\begin{array}{c}\text { R. Spitze H. rauhes } \\
\text { Atmen, noch etwas } \\
\text { Knacken. } \\
\text { L. Spitze rauhes } \\
\text { Atmen }\end{array}$ \\
\hline 34 & $\begin{array}{l}\text { F.', } \\
\text { Kaufmann }\end{array}$ & $\begin{array}{l}\text { Seit } 4 \text { Jahren links- } \\
\text { seit. Spitzenkatarrh, } \\
\text { Verschlimmerung } \\
\text { vor } 1 \text { Jahr. }-6 \text { w ö- } \\
\text { chentliche Kur in } \\
\text { - Davos. - Heiserkeit }\end{array}$ & 一 & $\begin{array}{l}\text { Über der R. Lunge } \\
\text { abgeschwächtes At- } \\
\text { men; U.-Lappenstar- } \\
\text { kes Rauschen. - } \\
\text { L. ebenso; ausser- } \\
\text { dem über der Spitze } \\
\text { schwaches Giemen }\end{array}$ & $\begin{array}{c}\text { Über beiden Spitzen } \\
\text { ves.-bronch. Atmen, } \\
\text { interponiertes Rau- } \\
\text { schen }\end{array}$ \\
\hline 35 & $\begin{array}{l}\text { D., } \\
43 \text { J., } \\
\text { Sekretär }\end{array}$ & $\begin{array}{l}\text { Vor } 6 \text { Jahren rechts- } \\
\text { seit. Lungenspitzen- } \\
\text { katarrh festgestellt. } \\
\text { Vor } 1 \text { Monat ge- } \\
\text { legentlich einer An- } \\
\text { gina wurdedie Lunge } \\
\text { untersucht u. krank } \\
\text { befunden }\end{array}$ & 一 & $\begin{array}{l}\text { R. Spitze rel. Dämpf. } \\
\text { mit Narbenatmen; } \\
\text { RH. neben Angulus } \\
\text { in der Tiefe dumpfe } \\
\text { feuchte Geräusche. } \\
\text { L. bis II. Rippe und } \\
\text { Spina Schallkür- } \\
\text { zung, Insp. etwas } \\
\text { scharf. Rhonchi in } \\
\text { diesen Grenzen nicht } \\
\text { zahlr, fein, feucht, } \\
\text { durch Husten deutl. } \\
\text { Rauschen beiders. }\end{array}$ & $\begin{array}{l}\text { Über den Spitzen } \\
\text { ves.-bronch. Atmen. } \\
\text { Keine Rhonchi; nur } \\
\text { LHU.leichtesPleura- } \\
\text { Schaben }\end{array}$ \\
\hline 36 & $\begin{array}{l}\text { O., } \\
19 \text { J., } \\
\text { Frl. }\end{array}$ & $\begin{array}{c}\text { Krank seit }{ }^{3} / 4 \text { Jahren. } \\
\text { Gewichtsverlust, } \\
\text { Husten u. Auswurf } \\
\text { (elastische Fasern } \\
\text { enthaltend, Tb.-Ba- } \\
\text { zillen nicht gefun- } \\
\text { den!) }\end{array}$ & - & $\begin{array}{l}\text { R. Sp. relat. Dämpf., } \\
\text { ves.-bronch. Atmen } \\
\text { obne Rhonchi }\end{array}$ & $\begin{array}{c}\text { Befund wie bei der } \\
\text { Aufvahme }\end{array}$ \\
\hline
\end{tabular}




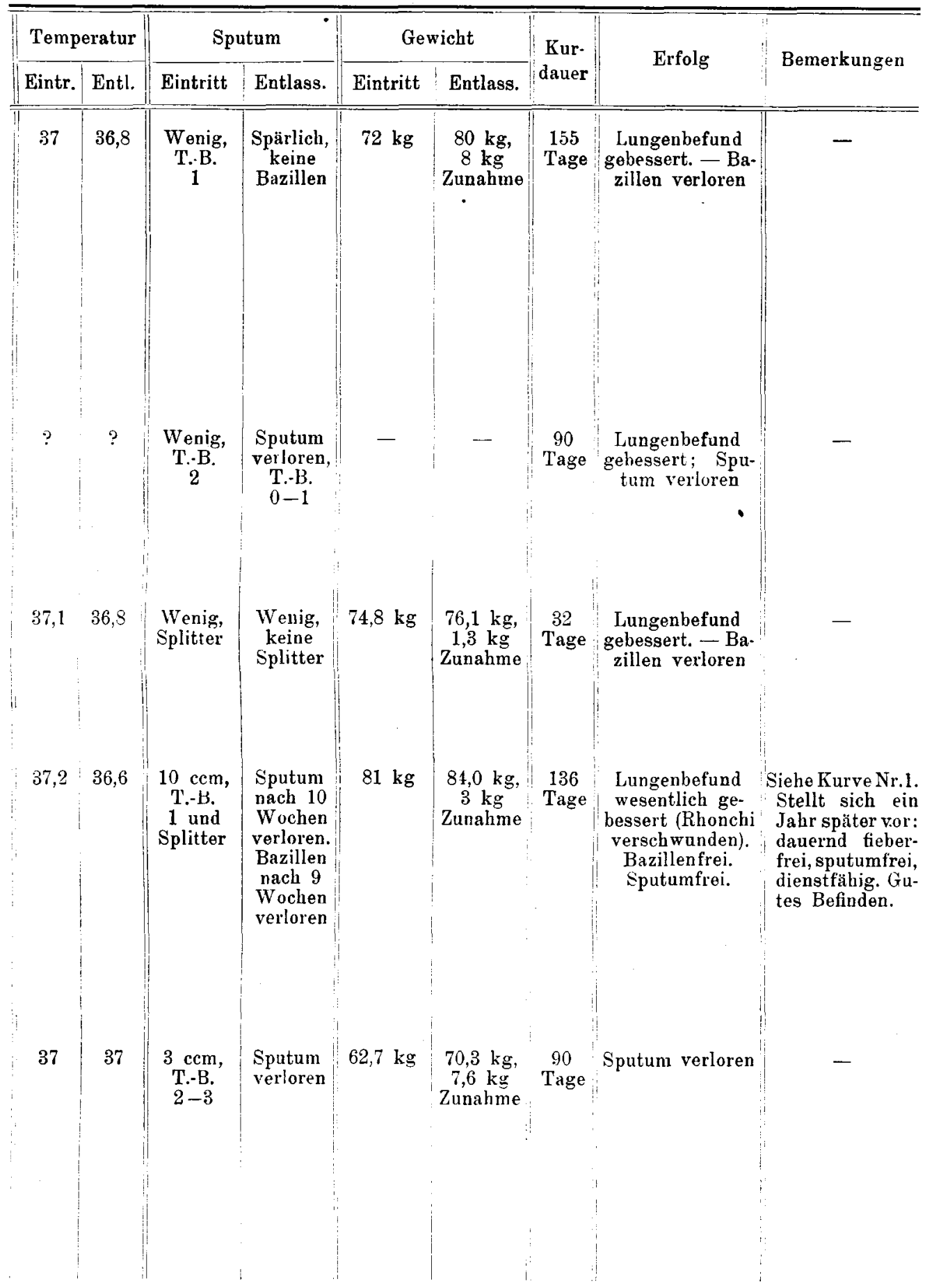

Beiträge zur Klinik der Tuberkulose. Bd, XVI. H. 4. 


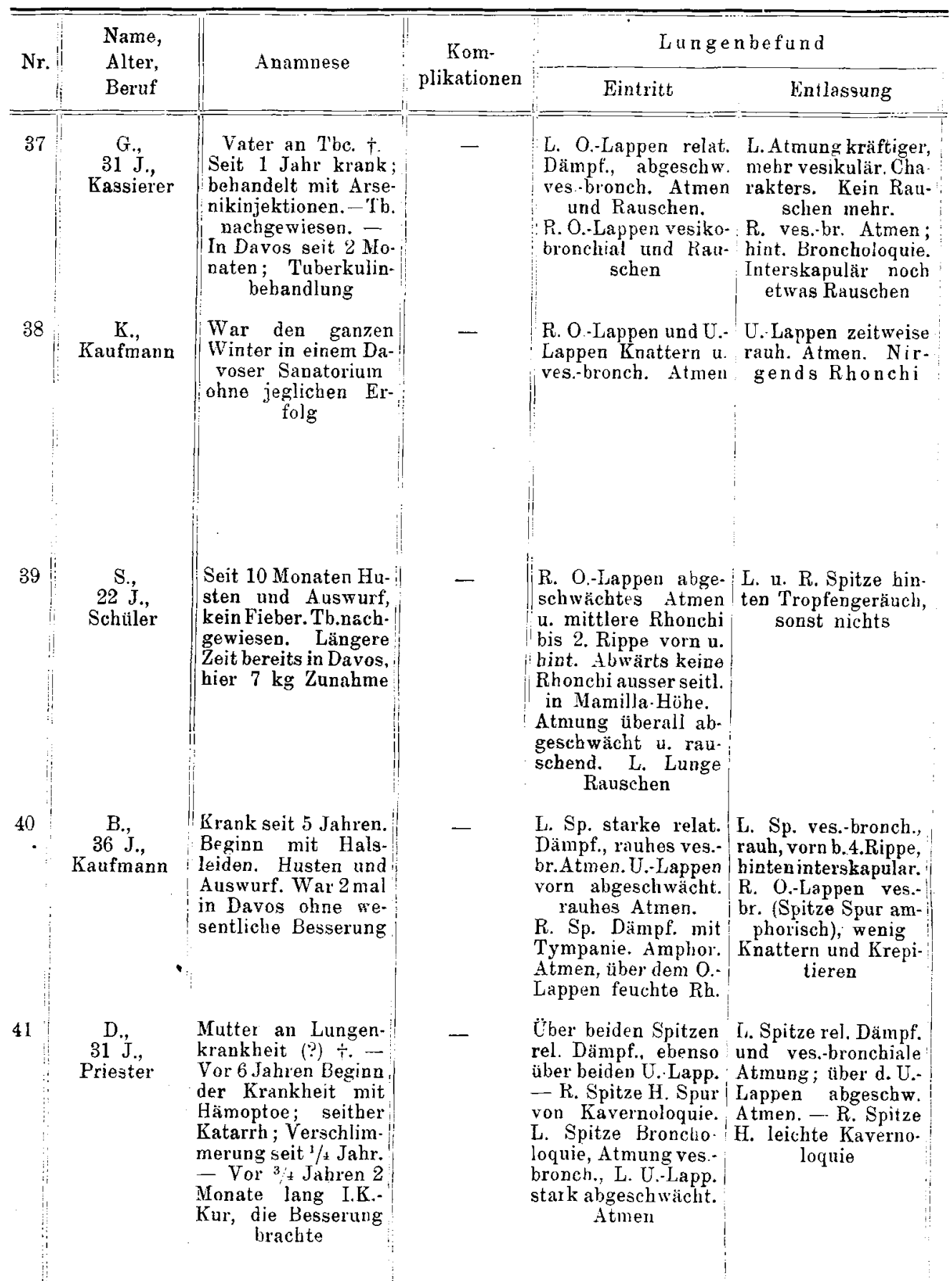




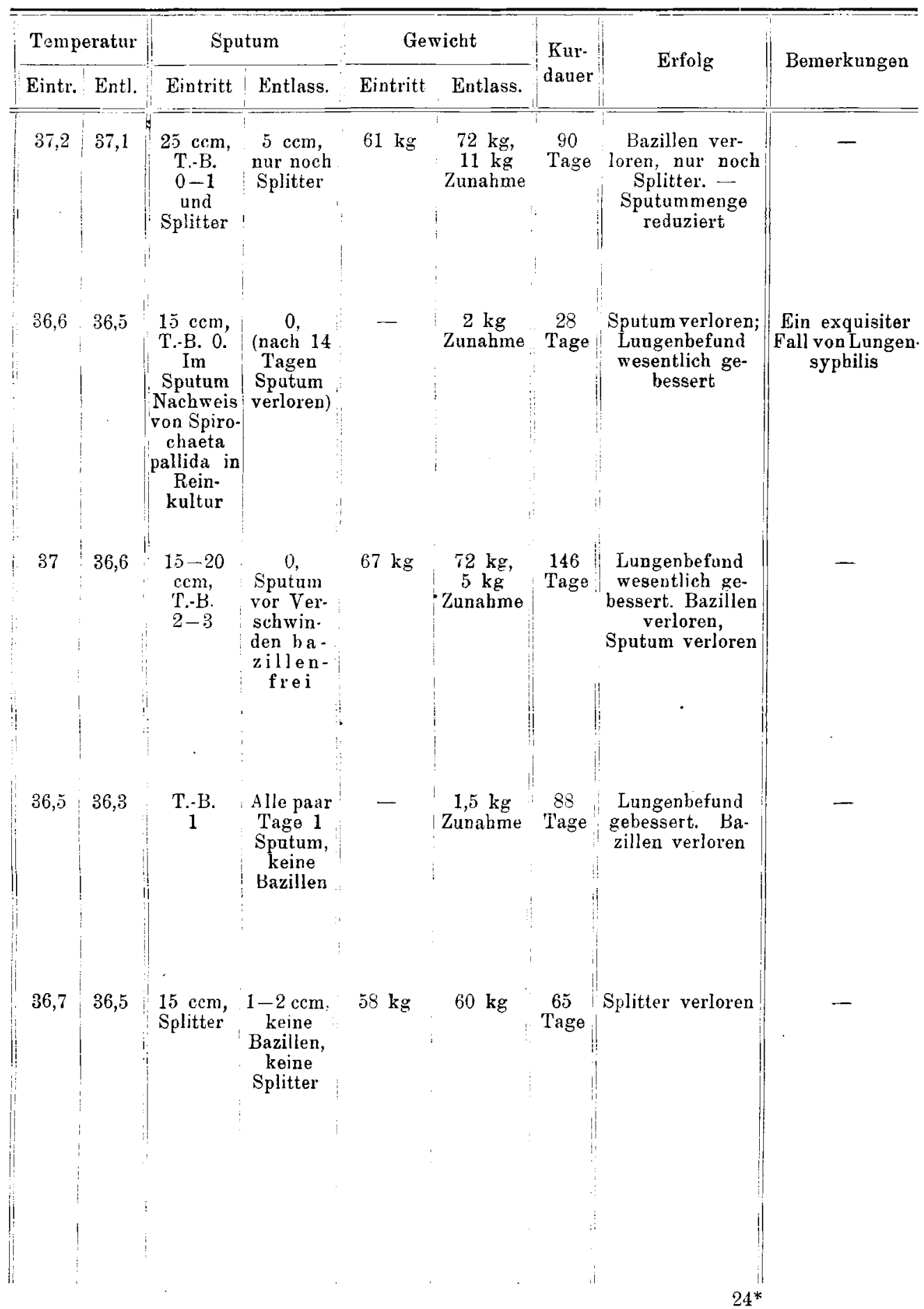


Etappen-

\begin{tabular}{|c|c|c|c|c|c|}
\hline \multirow{2}{*}{ Nr. } & \multirow{2}{*}{$\begin{array}{l}\text { Name, } \\
\text { Alter, } \\
\text { Beruf }\end{array}$} & \multirow{2}{*}{ Anamnese } & \multirow{2}{*}{$\begin{array}{c}\text { Kom. } \\
\text { plikationen }\end{array}$} & \multicolumn{2}{|c|}{ Lungenbefund } \\
\hline & & & & Eintritt & Entlassung \\
\hline \multirow[t]{2}{*}{42} & $\begin{array}{l}\text { A., } \\
\text { 36 J., } \\
\text { Kaufmann }\end{array}$ & $\begin{array}{l}\text { I. Etappe: } \\
\text { Vor } 8 \text { Jahren Syphi- } \\
\text { lis. Lungenkrankseit } \\
8 \text { Monaten. Spitzen- } \\
\text { affekt. festgestellt. } \\
\text { Keine Bazillen ge- } \\
\text { funden. Vor 5 Jabren } \\
\text { Pleuritis. Mit Tuber- } \\
\text { kulin vorbehandelt }\end{array}$ & 一 & $\begin{array}{l}\text { L. Sp. unbestimmtes } \\
\text { rauschendes Insp., } \\
\text { Exsp. ves, - bronch. } \\
\text { mit Knattern, relat. } \\
\text { Dämpf. - Sonst } \\
\text { überall Rauschen u. } \\
\text { unbestimmtes, z. T. } \\
\text { nahezu aufge- } \\
\text { hobenes Atmen }\end{array}$ & $\begin{array}{c}\text { Keine Rhonchi mehr. } \\
\text { Rauschen noch vor- } \\
\text { handen,aber weniger } \\
\text { stark }\end{array}$ \\
\hline & & $\begin{array}{l}\text { II. E t appe: } \\
10 \text { Monate später. } \\
\text { Immer noch leicht } \\
\text { ermüdbar }\end{array}$ & & $\begin{array}{l}\text { L. Sp, rel. Dämpf., } \\
\text { ves. bronch, rauhes } \\
\text { Atmen, Rauschen }\end{array}$ & $\begin{array}{l}\text { L. Sp. ves.-bronch., } \\
\text { obne Rauhigkeiten, } \\
\text { uberall an Stelle des } \\
\text { rauschenden Atmens } \\
\text { eine Hälfte der In- } \\
\text { spirationsphase ves. } \\
\text { Atmen. Atmung im } \\
\text { algem. ausgiebiger }\end{array}$ \\
\hline \multirow[t]{2}{*}{43} & $\begin{array}{l}\text { v. P., } \\
33 \text { J., } \\
\text { Frau }\end{array}$ & $\begin{array}{c}\text { I. Et appe: } \\
\text { Seit } 5 / 4 \text { Jahren in } \\
\text { ärztl. Behandlung } \\
\text { wegenLungenkrank- } \\
\text { beit. - Längere Zeit } \\
\text { Temperaturen bis } \\
\text { 37,5 und darüber; } \\
\text { unter PE und TBE } \\
\text { Speng ler wurde } \\
\text { die Temp. fast nor- } \\
\text { mal und der Lungen- } \\
\text { befund besserte sich. } \\
\text { Häuslicher Ver- } \\
\text { hältnisse wegen vom } \\
\text { Arzt nach Davos ge- } \\
\text { schickt }\end{array}$ & $\begin{array}{l}\text { Abszess I. in- } \\
\text { terskapular u. } \\
\text { erin eröftheter } \\
\text { Abszess am } \\
\text { L. Vorderarm. } \\
\text { (Nach Injektion } \\
\text { von Emulsion } \\
\text { entstanden) }\end{array}$ & $\begin{array}{l}\text { R. Spitze V. und H. } \\
\text { u. interskapularves.- } \\
\text { bronch. Atmen, ver- } \\
\text { einzelt. Knattern. } \\
\text { L. Sp. ves.-bronch., } \\
\text { rauh }\end{array}$ & $\begin{array}{l}\text { Rpitze unreines } \\
\text { Atmen }\end{array}$ \\
\hline & & $\begin{array}{l}\text { II. E tappe: } \\
\text { War } 6 \text { Monate zu } \\
\text { Hause, ohne sich zu } \\
\text { schonen. In letzter } \\
\text { Zeit wieder Tempe- } \\
\text { raturerhöhung bis } \\
\text { 37,4. Etwas Auswurf }\end{array}$ & & $\begin{array}{l}\text { R. Sp. rel. Dämpf., } \\
\text { vereinzelte, trockene } \\
\text { Rhonchi }\end{array}$ & $\begin{array}{l}\text { Rhonchi verschwun. } \\
\text { den }\end{array}$ \\
\hline \multirow[t]{2}{*}{44} & $\begin{array}{l}\text { W., } \\
21 . ., \\
\text { Fräulein }\end{array}$ & $\begin{array}{l}\text { I. E t a ppe. } \\
\text { Mutter an Lungen- } \\
\text { Brustfellentzündung } \\
\text { gest. Seit } 3 \text { Monaten } \\
\text { Husten und Auswurf }\end{array}$ & - & $\begin{array}{l}\text { R. Oberlappen ves.- } \\
\text { bronch. Atmen, rauh, } \\
\text { keine Rhonchi }\end{array}$ & $\begin{array}{l}\text { R. hinten über der } \\
\text { Sp. ves.-broneh. und } \\
\text { etwas Knacken. }\end{array}$ \\
\hline & & $\begin{array}{l}\text { II. Etap pe. } \\
10 \text { Monate später. } \\
\text { Inzwisch. Influenza. } \\
\text { Vor zwei Monaten } \\
\text { durch den Hausarzt } \\
\text { noch Bazillen nach- } \\
\text { gewiesen. }(0-1)\end{array}$ & & $\begin{array}{l}\text { R. Sp. rel.Dämpfung } \\
\text { res.-bronch., rauh, } \\
\text { ohne Rhonchi }\end{array}$ & $\begin{array}{l}\text { R. Sp. rel. Dämpfung } \\
\text { abgeschwächt. ves. } \\
\text { bronch. Atmen }\end{array}$ \\
\hline
\end{tabular}


fälle.

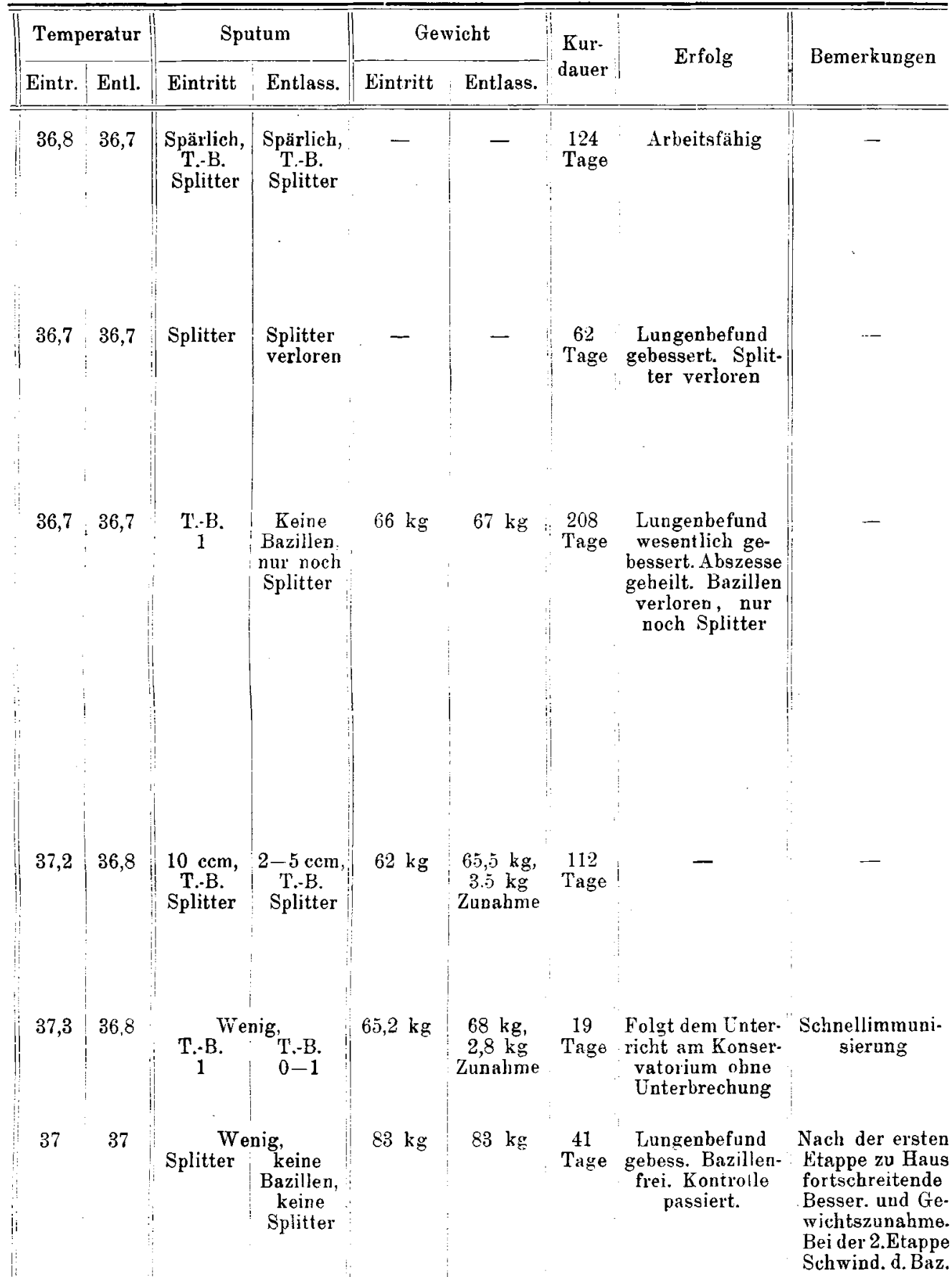


Klasse I.

\begin{tabular}{|c|c|c|c|c|c|}
\hline \multirow{2}{*}{ Nr. } & \multirow{2}{*}{$\begin{array}{l}\text { Name, } \\
\text { Alter, } \\
\text { Beruf }\end{array}$} & \multirow{2}{*}{ Anamnese } & \multirow{2}{*}{$\begin{array}{c}\text { Kom- } \\
\text { plikationen }\end{array}$} & \multicolumn{2}{|c|}{ Lungenbefund } \\
\hline & & & & Eintritt & Entlassung \\
\hline 45 & $\begin{array}{c}\text { T., } \\
\text { 33 J., } \\
\text { Ingenieur }\end{array}$ & - & - & $\begin{array}{l}\text { Über beid.O.-Lappen, } \\
\text { besond. vorn, leises } \\
\text { feines Knattern }\end{array}$ & $\begin{array}{l}\text { Über beid. O--Lappen } \\
\text { Alveolar- (Eröff- } \\
\text { nungs-) Knattern }\end{array}$ \\
\hline 46 & $\begin{array}{l}\text { B., } \\
\text { 33 J., } \\
\text { Frau }\end{array}$ & $\begin{array}{l}\text { Bruder lungenkrank. } \\
\text { Krank seit } 3 \text { Wochen. } \\
\text { Husten u. Auswurf. } \\
\text { Schmerzen über dem } \\
\text { ganzen Rücken, Ap- } \\
\text { petit mässig, Schlaf } \\
\text { schlecht, Herz- } \\
\text { klopfen }\end{array}$ & Neurasthenie & $\begin{array}{l}\text { R. u. L. Spitze rel. } \\
\text { Dämpf., ves.-bronch. } \\
\text { bis rauhes Atmen, } \\
\text { hinten beiders. Spur } \\
\text { von Kavernoloquie, } \\
\text { über den U. Lappen } \\
\text { etwas Rauschen }\end{array}$ & $\begin{array}{l}\text { L. Spitze u. infra- } \\
\text { klavikular rauhes, } \\
\text { ves.-bronch. Atmen, } \\
\text { ebenso hinten oben }\end{array}$ \\
\hline 47 & $\begin{array}{l}\text { St., } \\
22 \text { J., } \\
\text { Frl. }\end{array}$ & $\begin{array}{l}\text { Vor } 1 \text { Jahr Lungen- } \\
\text { entzündung, darnach } \\
\text { Katarrb. Längere } \\
\text { Zeit bettlägerig. - } \\
\text { Appetitlosigkeit, } \\
\text { Magenstörung, Ner- } \\
\text { vosität }\end{array}$ & - & $\begin{array}{l}\text { R. O.- u. M.-Lappen } \\
\text { Pottenger positiv, } \\
\text { bes. RH. uber der } \\
\text { Spitze stark ausge- } \\
\text { sprochen.-R.Spitze } \\
\text { V.tympanit., H. rel. } \\
\text { Dämpt.-R.O.- u.M.- } \\
\text { Lappen ves.-bronch. } \\
\text { raub, mit feinen, } \\
\text { knack. Rhonchis. - } \\
\text { L. Sp. ves.-bronch.; } \\
\text { beide U. Lappen } \\
\text { scharf vesikulär }\end{array}$ & $\begin{array}{l}\text { R. Spitze gedämpft } \\
\text { tympanit., V. ves.- } \\
\text { bronch. amphor. At- } \\
\text { men; ohne Husten, } \\
\text { keine Rhonchi, nach } \\
\text { Husten klingendes } \\
\text { Knacken }\end{array}$ \\
\hline 48 & $\begin{array}{c}\text { A., } \\
49 \text { J., } \\
\text { Ingenieur }\end{array}$ & $\begin{array}{l}\text { Ein Bruder an } \\
\text { Phthise gestorben, } \\
\text { eineSch westertuber- } \\
\text { kulös. Seit } 25 \text { Jahren } \\
\text { krank, Husten und } \\
\text { Auswurf. T.-B. schon } \\
\text { damals nachge- } \\
\text { wiesen }\end{array}$ & - & $\begin{array}{l}\text { R. „Pottenger } 2-3 . \\
\text { Rippe. R. O. Lappen } \\
\text { rel. Dpt., Rauschen. } \\
\text { R. Spitze leises ves.- } \\
\text { bronch. Atmen, infra- } \\
\text { klavik. bis 4. Rippe } \\
\text { Knattern; hinten ob. } \\
\text { Kavernoloquie, iiber } \\
\text { O.- u. U.-Lappen ab- } \\
\text { geschw. Atmen. L. } \\
\text { ïberall Rauschen. } \\
\text { Über der L. Sp. Spur } \\
\text { von Kavernoloquie }\end{array}$ & $\begin{array}{l}\text { R. O--Lappen abge- } \\
\text { schwächtes, unbe- } \\
\text { stimmtes Atmen, } \\
\text { uber der Spitze vesi- } \\
\text { kulär, hinten weiter } \\
\text { abwärts ves.-bronch. } \\
\text { Nirge uds } \\
\text { Rhonchi }\end{array}$ \\
\hline 49 & $\begin{array}{l}\text { L., } \\
22 \mathrm{~J} . \\
\text { Frl. }\end{array}$ & $\begin{array}{l}\text { Vater an Tbe. } \dagger \text {, } \\
\text { Mutter tuberkulös. - } \\
\text { Mit } 6 \text { Jahren Lungen- } \\
\text { entzündung; } \\
\text { Neigung zu Katar- } \\
\text { rhen. - Seit } 3 \text { Wo- } \\
\text { chen Husten n. Aus- } \\
\text { wurf, der T.-B. ent- } \\
\text { hält }\end{array}$ & - & $\begin{array}{l}\text { Über beiden Spitzen } \\
\text { Dämpfung, R. L.; } \\
\text { rechte Seite schleppt } \\
\text { etwas. - R. Spitze } \\
\text { rauhes, ves.-bronch. } \\
\text { Atmen, vereinzelte } \\
\text { feine Rh., V. U. H. } \\
\text { L.O-Lappen Rausch. }\end{array}$ & $\begin{array}{l}\text { R. Spitze vorn und } \\
\text { hinten ves.bronch. } \\
\text { ohne Rhonchi }\end{array}$ \\
\hline
\end{tabular}


Stadium 3.

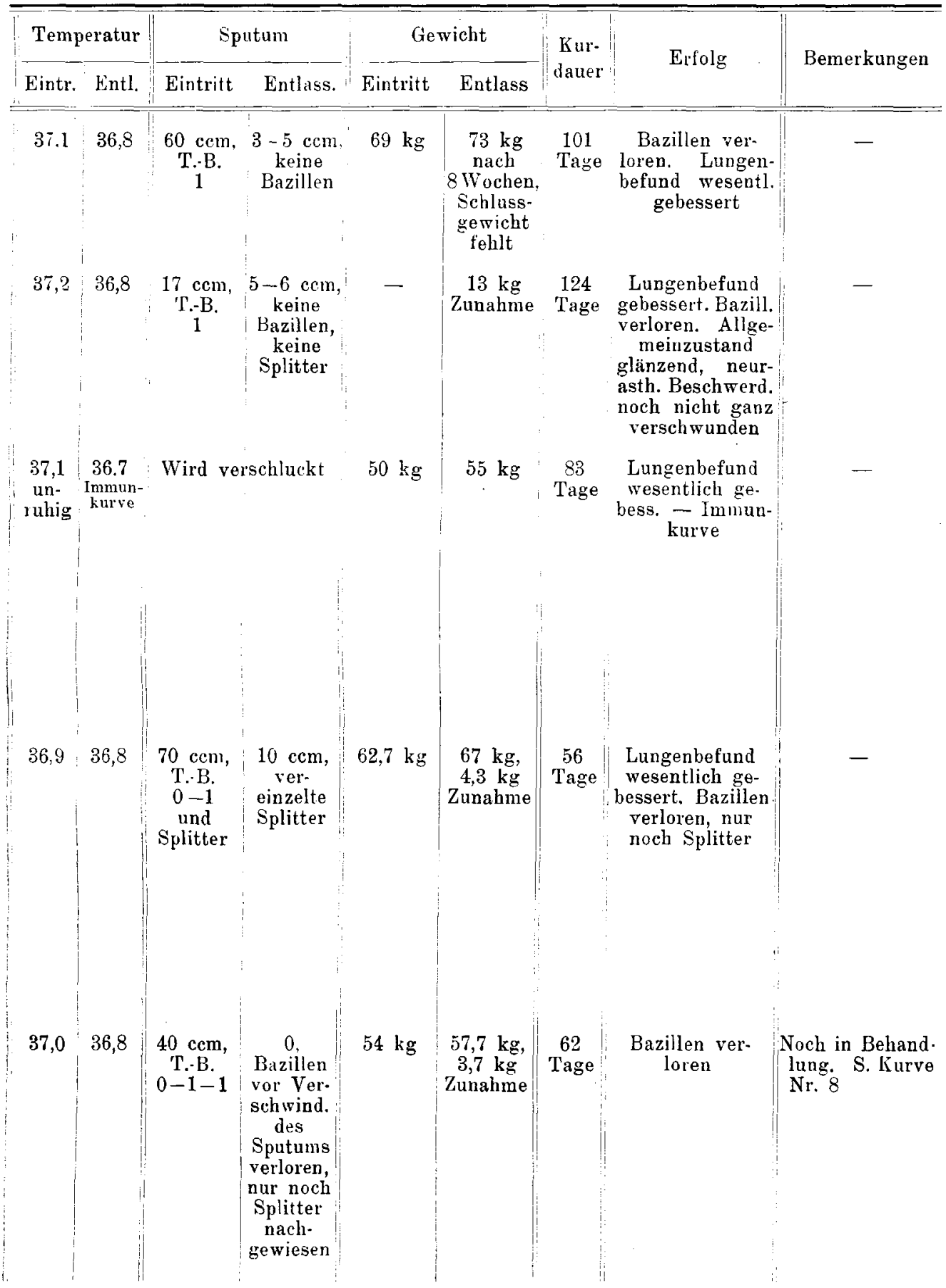




\begin{tabular}{|c|c|c|c|c|c|}
\hline \multirow{2}{*}{ Nr } & \multirow{2}{*}{$\begin{array}{l}\text { Name, } \\
\text { Alter, } \\
\text { Beruf }\end{array}$} & \multirow{2}{*}{ Anamnese } & \multirow{2}{*}{$\begin{array}{c}\text { Kom- } \\
\text { plikationen }\end{array}$} & \multicolumn{2}{|c|}{ Lungenbefund } \\
\hline & & & & Eintritt & Entlassung \\
\hline 50 & $\begin{array}{l}\text { Ch., } \\
42 \mathrm{~J} ., \\
\text { Agent }\end{array}$ & $\begin{array}{l}\text { Vor } 8 \text { Jahren Er- } \\
\text { krankung des l. O.- } \\
\text { Lappens, in Görbers- } \\
\text { dorf geheilt entlass. } \\
\text { Wiedererkrankung } \\
\text { vor } / \pm \text { Jahren, Hä- } \\
\text { moptoe. Seit einigen } \\
\text { Monaten in Davos, } \\
\text { zeitweise fiebernd }\end{array}$ & - & $\begin{array}{l}\text { R. u. L. O.-Lappen } \\
\text { rel. Dämpf., über d. } \\
\text { O.-Lappen broncho- } \\
\text { amphor. Atm., eben- } \\
\text { so r. M.-Lappen. } \\
\text { L. Spitze und inter- } \\
\text { skap. Atmen ves.- } \\
\text { bronch. u. Knattern. } \\
\text { Über M.-Lapp.grosse } \\
\text { u. kleine Rhonchi }\end{array}$ & $\begin{array}{l}\text { R. O. Lappen tymp. } \\
\text { gedämpft, broncho } \\
\text { amphor. Atraen mit } \\
\text { ganz leisen spärl. } \\
\text { Rhonchi. L. Spitze } \\
\text { u. interskap. Atmen } \\
\text { ves.-bronch., selten } \\
\text { ein Rhonchus }\end{array}$ \\
\hline 51 & $\begin{array}{l}\text { K., } \\
33 \stackrel{J_{*}}{ } \\
\text { Lehrerin }\end{array}$ & $\begin{array}{c}\text { Vor } 6 \text { Jahren Pleu- } \\
\text { ritis exsudat. dextra. } \\
\text { Vor } 4 \text { Wochen Hä- } \\
\text { moptoe }\end{array}$ & $\begin{array}{l}\text { Laryngitis } \\
\text { tuberculosa. } \\
\text { Rötung undVer- } \\
\text { dickung der } \\
\text { Stinmbănder, } \\
\text { Schwellung } \\
\text { der Aryknorpel. } \\
\text { Entlassung: } \\
\text { Keine Rötung, } \\
\text { keine Sehwel- } \\
\text { lung mehr, } \\
\text { Stimmbänder } \\
\text { leicht verdickt }\end{array}$ & $\begin{array}{l}\text { R. O.-Lappen ves.- } \\
\text { bronch. hinten halb- } \\
\text { klingende Rhonchi. } \\
\text { L.Spitze ves.-bronch. }\end{array}$ & $\begin{array}{l}\text { R. u. L. Spitze ves.- } \\
\text { bronch. R. Spitze } \\
\text { mit grob. Knacken, } \\
\text { R. M.-Lappen Atm. } \\
\text { rauh, ves.bronch. } \\
\text { L. keine Nebenge- } \\
\text { räusche }\end{array}$ \\
\hline 52 & 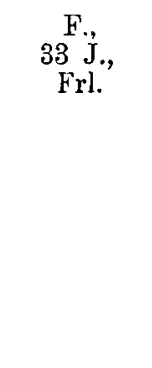 & $\begin{array}{l}\text { Schwester lungen- } \\
\text { krank; Beginn der } \\
\text { Krankheit vor } 2 \text { J. } \\
\text { mit Husten u. Aus- } \\
\text { wurf u. subfebriler } \\
\text { Temp.; seit } 2 \text { Mon. } \\
\text { Verschlechterung. } \\
\text { MitI.K.vorbehandelt } \\
\text { mit gutem Erfolg. } \\
\text { (Ans Familienrück- } \\
\text { sichten v. Arzt nach } \\
\text { Davos geschickt) }\end{array}$ & i! $\quad-\quad$ & $\begin{array}{l}\text { Spitz rel. gedämpft; } \\
\text { leises ves.-bronch. } \\
\text { Atmen, bes. rechts }\end{array}$ & $\begin{array}{l}\text { R. Spitze rel. Dämpf., } \\
\text { keine Rhonchi; leises } \\
\text { ves. -bronch. Atmen. } \\
\text { - L. Spitze ves.- } \\
\text { bronch. Atmen }\end{array}$ \\
\hline 53 & $\begin{array}{l}\text { E., } \\
31 \text { J., } \\
\text { Kranken- } \\
\text { schwester }\end{array}$ & $\begin{array}{l}\text { Vor } 1 \text { Jahr Pleuritis. } \\
\text { L. Spitzonkatarrh } \\
\text { festgestellt. - T. B. } \\
\text { nachgewiesen. } \\
\text { Vattigkeit; Schlaf u. } \\
\text { Appetit schlecht }\end{array}$ & - & $\begin{array}{l}\text { L. Lunge schleppt; } \\
\text { rel. Dämpf. überbeid. } \\
\text { O.-Lappen, R. > L. } \\
\text { R. Spitze vereinzelt. } \\
\text { Knacken n. Husten. } \\
\text { L. Spitze V. u. H. } \\
\text { gröb. leise Rhonchi. } \\
\text { Abgeschw.Atm. über } \\
\text { den U.-Lappen }\end{array}$ & $\begin{array}{l}\text { Schallkürzung über } \\
\text { beid. Spitzen R. }>\text { L. } \\
- \text { L. O.-Lappen leise } \\
\text { Rhonchi. - R. Spitze } \\
\text { vereinzelt. Knacken } \\
\text { nach Husten, - LU. } \\
\text { abgeschw. Atmen }\end{array}$ \\
\hline 54 & $\begin{array}{l}20 \stackrel{\text { J., }}{\text { J., }} \\
\text { Schüler }\end{array}$ & $\begin{array}{l}\text { Mutter an Tbc. } \\
\text { Krank seit } 3 \text { Jahren } \\
\text { vor } 3 \text { Monaten Hä- } \\
\text { moptoe, seither Ver- } \\
\text { schlimmerung } \\
\end{array}$ & - & $\begin{array}{l}\text { L. O.-Lapp. „Fotten- } \\
\text { ger , schleppt wenig; } \\
\text { relat. Dämpf., ves.- } \\
\text { bronch. mit knatt. } \\
\text { Rhonchis; hinten } \\
\text { Rauhigkeiten. L. U.- } \\
\text { Lappen ves.-bronch., } \\
\text { abgeschwächt. leises } \\
\text { Knattern. R. inter- } \\
\text { skapular Knattern }\end{array}$ & $\begin{array}{l}\text { L.Spitze rel. Dämpf., } \\
\text { ves.-bronch. Atmen; } \\
\text { O.-Lappen knack. } \\
\text { Rhonchi, - R. inter- } \\
\text { skap. feinste knack. } \\
\text { Rhonchi }\end{array}$ \\
\hline
\end{tabular}




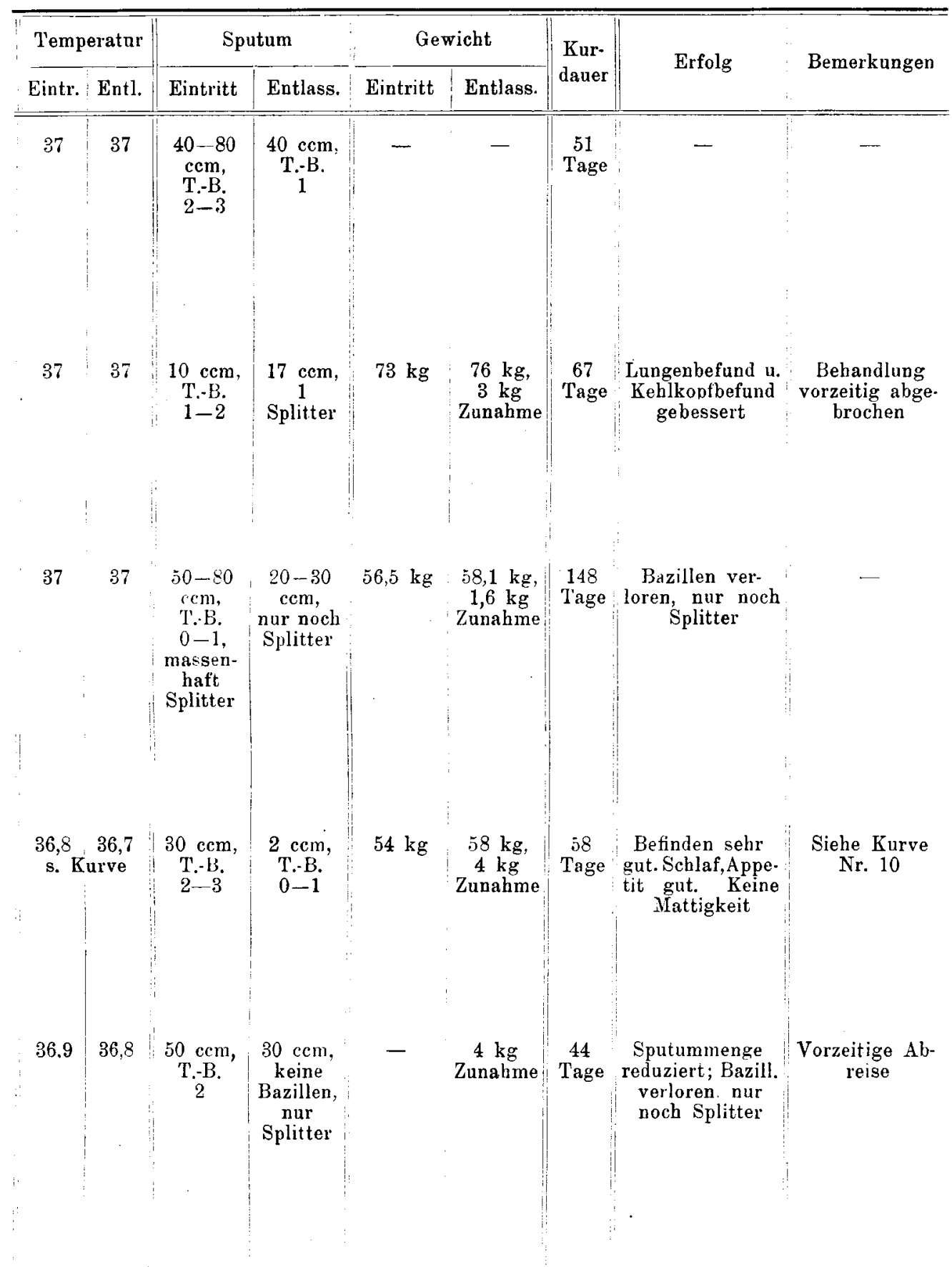




\begin{tabular}{|c|c|c|c|c|c|}
\hline \multirow{2}{*}{ Nr. } & \multirow{2}{*}{$\begin{array}{l}\text { Name, } \\
\text { Alter, } \\
\text { Beruf }\end{array}$} & \multirow{2}{*}{ Anamnese } & \multirow{2}{*}{$\begin{array}{l}\text { Kom- } \\
\text { plikationen }\end{array}$} & \multicolumn{2}{|c|}{ Lungenbefund } \\
\hline & & & & Eintritt & Entlassung \\
\hline 55 & $\begin{array}{l}\text { Sch., } \\
24 \text { J., } \\
\text { Maler }\end{array}$ & $\begin{array}{c}\text { Vor } 1 / 2 \text { Jahr nach } \\
\text { Influenza Husten, } \\
\text { Blutungen, Nacht. } \\
\text { schweisse.T.-B.nach- } \\
\text { gewiesen. } \\
\text { Friuher Syphilis }\end{array}$ & - & $\begin{array}{l}\text { R. O. Lappen st. rel. } \\
\text { Dämpf. R. Spitze } \\
\text { ves.-bronch. Exsp. } \\
\text { stark verlängert u. } \\
\text { verschärtt.Rauschen } \\
\text { über beiden Lungen }\end{array}$ & $\begin{array}{l}\text { R. O.-Lappen st. rel. } \\
\text { Dämpf. Sp. bronch. } \\
\text { amphor. A tmen, zäh. } \\
\text { Knarren. L̈ber dem } \\
\text { abrigen Oberlappen } \\
\text { ves.-bronch. Atmen } \\
\text { obne Rhonchi. }\end{array}$ \\
\hline 56 & $\begin{array}{l}\text { P., } \\
21 \text { J., } \\
\text { Student }\end{array}$ & $\begin{array}{l}\text { Seit } 1 \text { Jahr krank, } \\
\text { Initialhämoptoe. - } \\
\text { Mit Beraneck in } \\
\text { Lausanne behandelt }\end{array}$ & - & $\begin{array}{l}\text { L. Spitze schleppt } \\
\text { etwas; Spur rel. } \\
\text { Dämpt.; ves.-bronch. } \\
\text { Atmen rauh vorne, } \\
\text { hinten gleichfalls mit } \\
\text { vereinzelt. Knattern } \\
\text { nach Husten. R. } \\
\text { Spitze unreines, ver- } \\
\text { schiedenes Atmen }\end{array}$ & $\begin{array}{l}\text { L. Spitze vorn, R. } \\
\text { Spitze hinten eine } \\
\text { Spur Knacken }\end{array}$ \\
\hline 57 & $\begin{array}{l}21 \text { G., } \\
\text { Zahn- } \\
\text { techniker }\end{array}$ & $\begin{array}{l}\text { Mutter an Lungen- } \\
\text { karzioom } \dagger .- \\
\text { Husten u. Auswurf } \\
\text { seit } 15 \text { Jahren. - } \\
\text { Hämoptoe } 3 \text { mal, die } \\
\text { letzte in diesem Jahr }\end{array}$ & - & $\begin{array}{l}\text { L. über der ganzen } \\
\text { Lunge Knattern, be- } \\
\text { sonders seitlich und } \\
\text { basal. Giemen und } \\
\text { rauhes Atmen. R. } \\
\text { Lunge Rauschen }\end{array}$ & $\begin{array}{l}\text { Über den U.-Lappen } \\
\text { etwas Rauschen. - } \\
\text { Nirgends Rhonchi }\end{array}$ \\
\hline 58 & $\begin{array}{l}\text { B., } \\
33 . \mathbf{J}_{.}, \\
\text {Offizier }\end{array}$ & - & - & 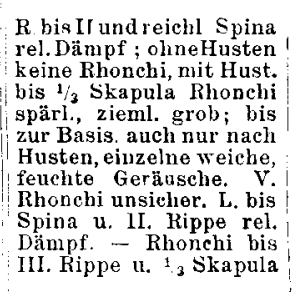 & $\begin{array}{l}\text { R. O.-Lappen rel. } \\
\text { Dämpf., ves.-bronch. } \\
\text { Atmen, rauh ohne } \\
\text { Rlonchi. L. Spitze } \\
\text { wie R. }\end{array}$ \\
\hline 59 & $\begin{array}{l}\text { R., } \\
\text { 33 J., } \\
\text { Korrespon- } \\
\text { dent }\end{array}$ & $\begin{array}{c}\text { Seit } 8 \text { Jahren magen- } \\
\text { leidend. Hyperazidi- } \\
\text { tät festgest. }(70 \%) \text {. } \\
\text { Lungenerkrankung } \\
\text { vor einer Woche } \\
\text { nachgewiesen. } \\
\text { Ständige Magenbe- } \\
\text { schwerden u. Appe- } \\
\text { titmangel }\end{array}$ & $\begin{array}{c}\text { Gastritis } \\
\text { chron }\end{array}$ & $\begin{array}{l}\text { Über beiden Spitzen } \\
\text { rel. Dämpfung. } \\
\text { Rauschen }\end{array}$ & $\begin{array}{l}\text { R. Spitze rel.Dämpf., } \\
\text { L. keine Dämpfung. } \\
\text { Rauschen bedeutend } \\
\text { weniger; fast überall } \\
\text { in der 1. Atmungs- } \\
\text { phase vesik. Atmen, } \\
\text { dann Rauschen }\end{array}$ \\
\hline 60 & $\begin{array}{l}\text { E., } \\
20 \stackrel{J}{\text { Student }}\end{array}$ & $\begin{array}{l}\text { Initialhämoptoe vor } \\
2 \text { Monaten; seither } \\
2 \text { weitere Blutungen. } \\
\text { T.-B. nicht gefunden. } \\
\text { Morgens Husten mit } \\
\text { Auswurf }\end{array}$ & - & 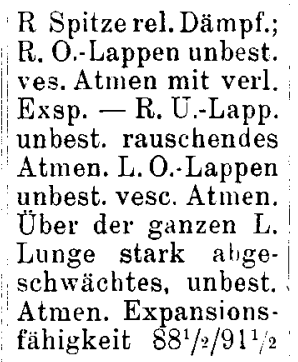 & - \\
\hline
\end{tabular}




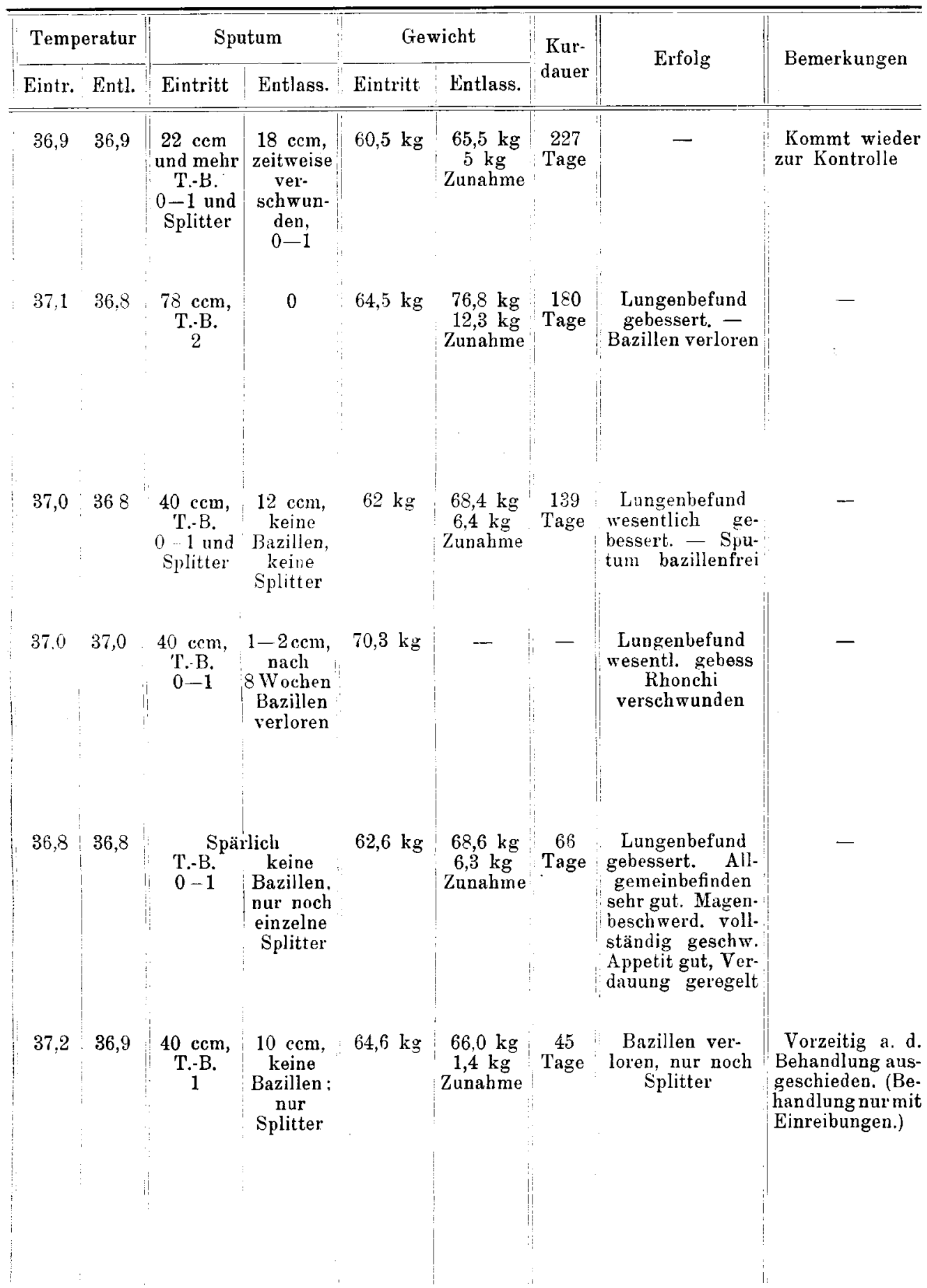


Etappen-

\begin{tabular}{|c|c|c|c|c|c|}
\hline \multirow{2}{*}{$\mathrm{Nr}$. } & \multirow{2}{*}{$\begin{array}{r}\text { Name, } \\
\text { Alter, } \\
\text { Beruf }\end{array}$} & \multirow{2}{*}{ Anamnese } & \multirow{2}{*}{$\begin{array}{c}\text { Kom- } \\
\text { plikationen }\end{array}$} & \multicolumn{2}{|c|}{ L $u n g e n b$ efund } \\
\hline & & & & Eintritt & Entlassung \\
\hline 61 & $\begin{array}{l}\text { G., } \\
28 \text { J., } \\
\text { Frl. }\end{array}$ & $\begin{array}{l}\text { I. Etappe: } \\
\text { Vater an Tbc. ge- } \\
\text { storben. Krank seit } \\
\text { 5Jahren, Beginn mit } \\
\text { starker Bronchitis. } \\
\text { Auswurf. Verschied. } \\
\text { klimatische Kuren } \\
\text { ohne Erfclg } \\
\text { Vorbehandelt mit } \\
\text { Tuberkulin } \\
\text { II. E t a p e: } \\
5 \text { Monate später }\end{array}$ & - & $\begin{array}{l}\text { R. Spitzerel. Dämpf., } \\
\text { ves.-bronch. Atmen, } \\
\text { Spur amphor., mit } \\
\text { feinen Rhonchi. L. } \\
\text { Spitze hinten idem, } \\
\text { R. O.-Lappen ves.- } \\
\text { bronch. ohneRhonchi } \\
\text { ausser hinten inter- } \\
\text { skapular, woselbst } \\
\text { Knattern } \\
\text { R. interskap. ves.- } \\
\text { bronch. bis bronch. } \\
\text { u. spärlich Knattern. } \\
\text { R. O.-Lappen u. L. } \\
\text { Spitze ves.-bronch. } \\
\text { Atmen, keine Rh. } \\
\text { L. hinten Giemen } \\
\text {. }\end{array}$ & $\begin{array}{l}\text { R. Spitze reines ves.- } \\
\text { bronch. Atmen. L. } \\
\text { Spitze nach Husten } \\
\text { Knarren,ves.-bronch. } \\
\text { amphor. Atmen. R. } \\
\text { intersk. rauh.Atmen, } \\
\text { ebenso L. hinten } \\
\text { unten }\end{array}$ \\
\hline
\end{tabular}

Klasse II.

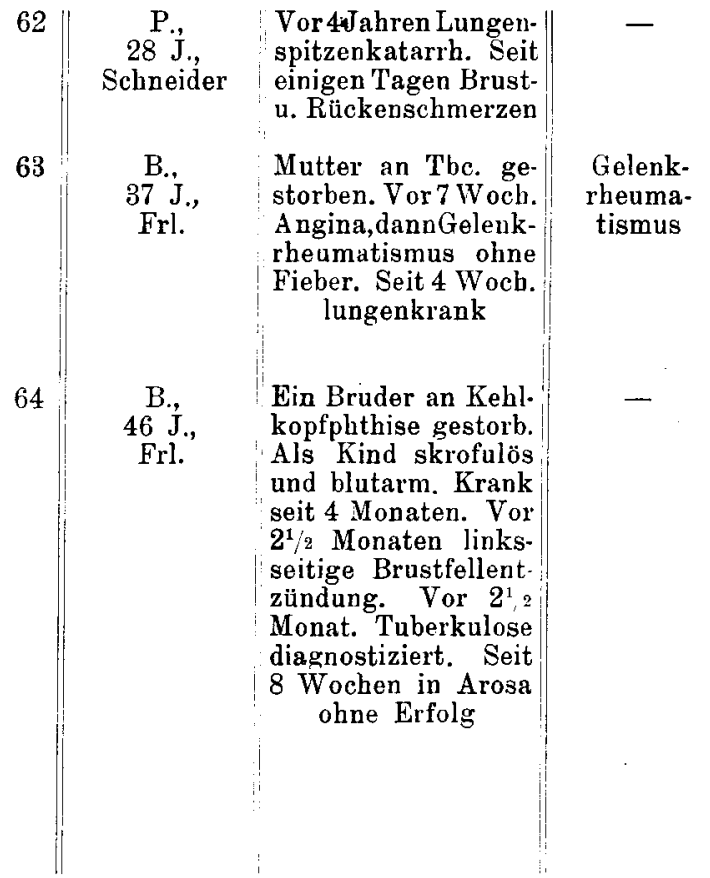

Rauschen über beiden Lungen

Rauschen noch vorhanden

R. Spitze stärker rel. R. Spitze rel. Dämpf. gedämpft als L. R. ves.-bronch. Atmen. Sp. Atmung scharf, L. Spitze reines ves. Exsp. stark verläng. L. Sp. rauhes Atmen. Über beiden Lungen bis Basis Rauschen

L. O.-Lappen rel. Beiderseits vesik. Dämpf., rauhes ves.- bronch. Atmen ohne bronch. Atmen. L. Rhonchi. R. U.Lapp. hinten über O.- u. U. ? hinten interskapular Lappen leise knatt. einekleine Stelle mit Rlonchi. R. O.-Lapp. feinstem Knacken rauhes scharfes ves.

Atmen, über dem

U. - Lappen hinten

Rauhigkeiten $u$. vereinzelte Rhonchi 
fall.

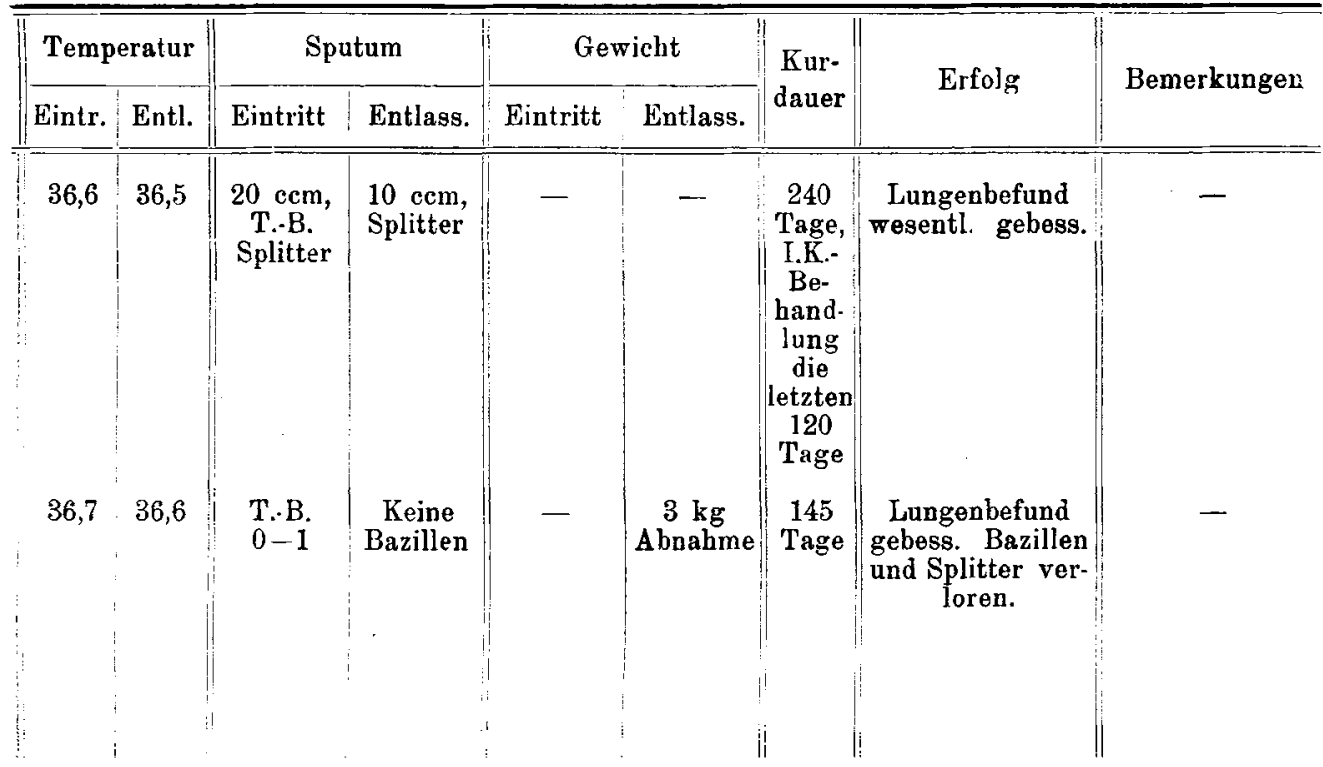

\section{Stadium 1.}

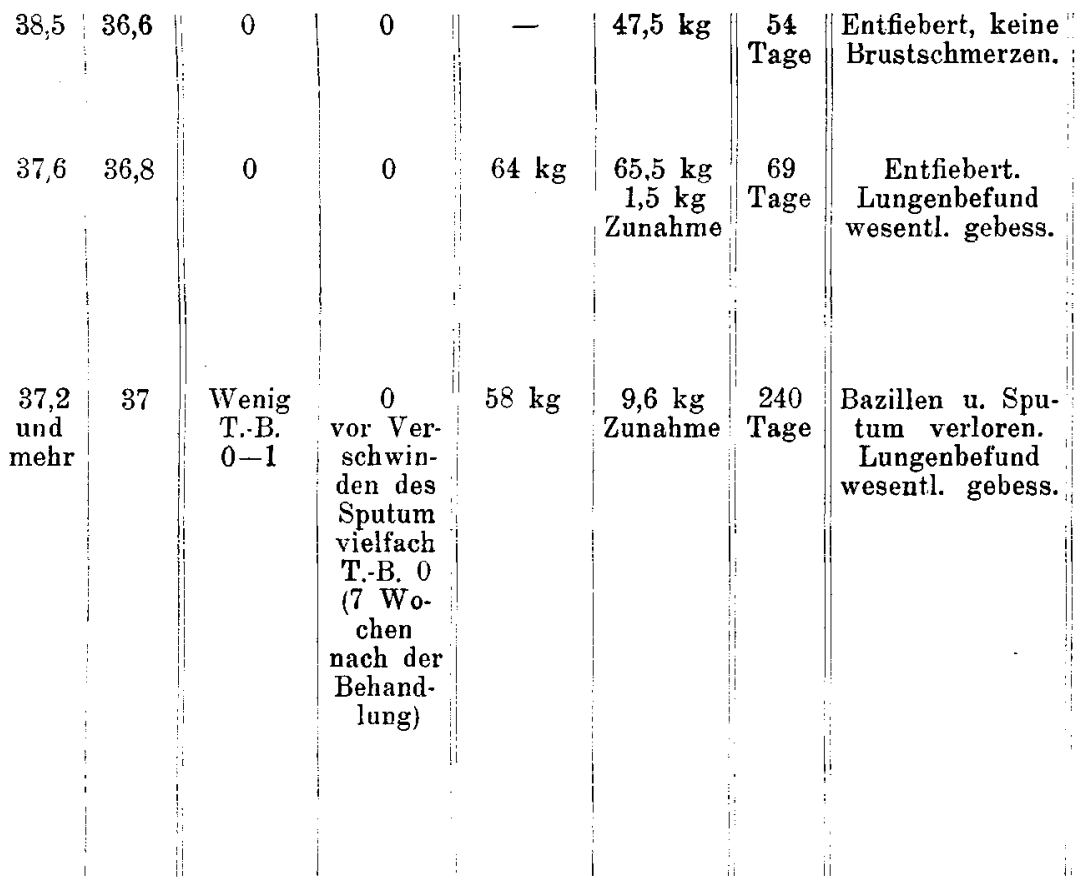




\begin{tabular}{|c|c|c|c|c|c|}
\hline \multirow{2}{*}{$\mathrm{Nr}$} & \multirow{2}{*}{$\begin{array}{r}\text { Name, } \\
\text { Alter, } \\
\text { Beruf }\end{array}$} & \multirow{2}{*}{ Anamnese } & \multirow{2}{*}{$\begin{array}{c}\text { Kom- } \\
\text { plikationen }\end{array}$} & \multicolumn{2}{|c|}{ Lungenbefund } \\
\hline & & & & Eintritt & Entlassung \\
\hline 65 & $\begin{array}{l}\text { E., } \\
23 \text { J., } \\
\text { Frl. }\end{array}$ & $\begin{array}{l}\text { Mutter, } 1 \text { Bruder, } 1 \\
\text { Sehwester an Tbe. } 1 \\
\text { Vor 3 Jahren Bronchitis, } \\
\text { trock. Husten, Fieber bis } \\
\text { 390, anschliess. Pleuritis } \\
\text { exsudativ dextr., Natht- } \\
\text { schweisse, Atemnot. } \\
\text { Sanatoriumsbehandlung } \\
\text { im Tiefland vor 1 Jahr, } \\
\text { als rel. geheilt entlassen. } \\
\text { Später Verschlimmerung } \\
\text { Husten, Fieber, rapide } \\
\text { Gewichtsabnahme }\end{array}$ & - & $\begin{array}{l}\text { R. O.-Lappen relat. } \\
\text { Dämpf., Atm. raah, } \\
\text { ves.bronch., über U.- } \\
\text { Lappen ebenso. L. } \\
\text { iutersk. vereinzeltes } \\
\text { Knattern. R. Spitze } \\
\text { hinten Spur von Ka- } \\
\text { vernoloquie. L.Spitze } \\
\text { leichte Schallkürz. } \\
\text { Atmung ves.-bronch. } \\
\text { rauh. }\end{array}$ & $\begin{array}{l}\text { Perkutorisch u. aus- } \\
\text { kultatorisch kaum } \\
\text { etwas nachzuweisen, } \\
\text { weder R. noch L. }\end{array}$ \\
\hline 66 & $\begin{array}{l}\text { B., } \\
23 \text { J." } \\
\text { Frl. }\end{array}$ & $\begin{array}{l}\text { Mutter an Tbc. } \dagger \text {. } \\
\text { Dyspnoe, Fieber, } \\
\text { starke Abmagerung. } \\
\text { Husten u. Auswurf }\end{array}$ & $\begin{array}{l}\text { Starke } \\
\text { Anämie }\end{array}$ & $\begin{array}{l}\text { R. O. Lapp. schleppt } \\
\text { stark, zieml. st. rel. } \\
\text { Dämpf. R. Spitze } \\
\text { Atm. bronch.-amph., } \\
\text { abwärts bis Basis } \\
\text { ves.-bronch. Überder } \\
\text { Spitze spärl. kleines } \\
\text { Knattern, abw. mittl. } \\
\text { Knattern vorn und } \\
\text { hinten. L. Spitze ves.- } \\
\text { bronch. rauh. }\end{array}$ & $\begin{array}{l}\text { R. O.-Lappen relat. } \\
\text { Dämpf., vorn Sp. } \\
\text { vereinz. leis. Knack., } \\
\text { Kavernoloquie, hint. } \\
\text { keine Rbonchi. L. } \\
\text { Sp. ves ves.-bronch. } \\
\end{array}$ \\
\hline 67 & $\begin{array}{l}\text { M., } \\
20 \stackrel{\text { J. }}{\text { Frl. }}\end{array}$ & $\begin{array}{l}\text { Pat. ist Einheimische; in } \\
\text { Davos gebor. 11. daselbst } \\
\text { wohnhaft In den letzten } \\
\text { Jahren Neigung zu Ka- } \\
\text { tarrben u. Halsentzünd. } \\
\text { Krank seit } 2 \text { Jahren. } \\
\text { Husten, Auswurf, Ge- } \\
\text { wichisabnahme, Nacht- } \\
\text { schweisse, Appetitlosig. } \\
\text { keit. - Tuberkulinkur ir } \\
\text { Davos weegen Brustfell- } \\
\text { entzünd unterbrochen }\end{array}$ & $\begin{array}{l}\text { Aorten- } \\
\text { stenose }\end{array}$ & $\begin{array}{l}\text { R. O.-Lappen Dämpf. } \\
\text { ves.- bronch. Atmen } \\
\text { mit Rhonchis. L. } \\
\text { hít. intersk. Dämpf. } \\
\text { rauhes ves.-bronch. } \\
\text { Atmen. Über beiden } \\
\text { Lungen Rauschen }\end{array}$ & - \\
\hline 68 & $\begin{array}{l}\mathrm{K} . \dot{\mathrm{J}} \\
4 \pm \text { Jurist }\end{array}$ & $\begin{array}{c}\text { Erbliche Belastung. } \\
\text { Seit } 2 \text { Monat Husten. } \\
\text { Hämoptoe }\end{array}$ & - & $\begin{array}{l}\text { R. O.-Lappen Dämpf. } \\
\text { rel. spär]. dumpfe } \\
\text { feuchte Rh. RVU. } \\
\text { u. R. S. pleurit. Reib. } \\
\text { I. Sp. Schallkiirzung } \\
\text { bis II. R Atmung un- } \\
\text { rein. LHU. Rauschen }\end{array}$ & $\begin{array}{l}\text { R. O.-Lappen u. L. } \\
\text { Sp. rel. Dämpf. ves.- } \\
\text { bronch. Atmen, nirg. } \\
\text { Rhonchi }\end{array}$ \\
\hline 69 & $\begin{array}{l}\text { S., } \\
37 \text { J., } \\
\text { Offizier }\end{array}$ & $\begin{array}{l}\text { Krank seit } 4 \text { Monat. } \\
\text { Sanatoriumskur in } \\
\text { Abbaz, dann } 8 \text { Woch } \\
\text { in Meran }\end{array}$ & $\ldots$ & $\begin{array}{l}\text { R. O.-Lappen ves.- } \\
\text { bronch. rauh, ab- } \\
\text { geschw. einzelne } \\
\text { Rhonchi }\end{array}$ & $\begin{array}{l}\text { R.Spitze ves.-bronch. } \\
\text { feines Knarren }\end{array}$ \\
\hline 70 & $\begin{array}{c}\text { Sch., } \\
18 \mathrm{~J} ., \\
\text { Kaufmann }\end{array}$ & - & - & $\begin{array}{l}\text { R. Sp, ves.bronch. } \\
\text { Atmen, Raubigkeit. } \\
\text { L. Spitze abgeschw. } \\
\text { rauhes Atmen. } \\
\text { Rauschen beiderseits }\end{array}$ & $\begin{array}{l}\text { R. Spitze reines ves.- } \\
\text { bronch. Atmen }\end{array}$ \\
\hline 71 & $\begin{array}{l}\text { H., } \\
\text { Frl. }\end{array}$ & $\begin{array}{l}\text { Husten, Fieber, Kopf. } \\
\text { schmerzen, Menstru- } \\
\text { ationsbeschwerden. }\end{array}$ & 一 & $\begin{array}{l}\text { R. O.-Lappen ves.- } \\
\text { bronch. Knacken } \\
\text { vorn u. hinten. }\end{array}$ & $\begin{array}{l}\text { R O.-Lappen ves. bis } \\
\text { ves.-bronch. Atmen, } \\
\text { keine Rhonchi und } \\
\text { keine Raubigkeiten }\end{array}$ \\
\hline
\end{tabular}




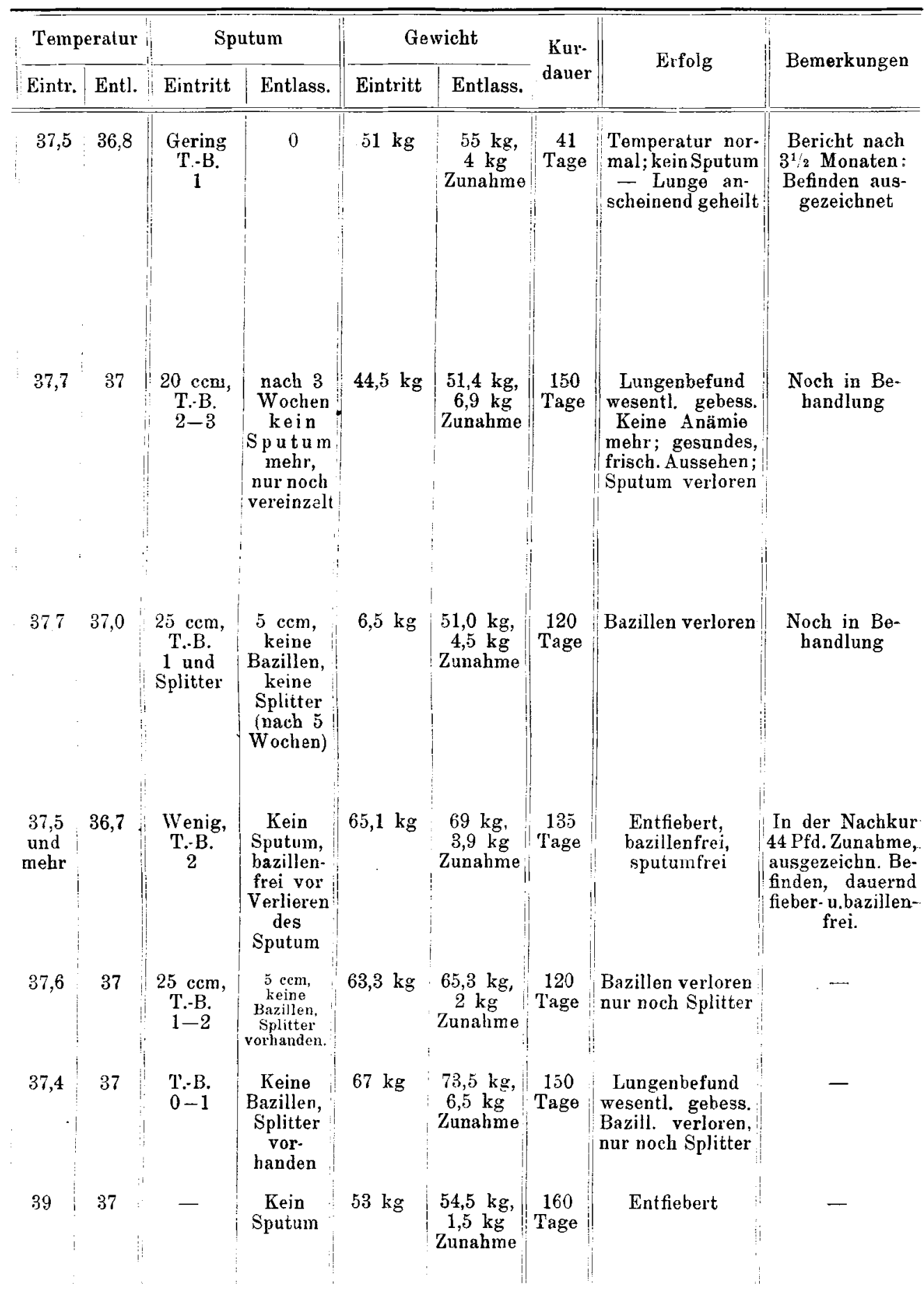




\begin{tabular}{|c|c|c|c|c|c|}
\hline \multirow{2}{*}{ Nr. } & \multirow{2}{*}{$\begin{array}{r}\text { Name, } \\
\text { Alter; } \\
\text { Beruf }\end{array}$} & \multirow{2}{*}{ Anamnese } & \multirow{2}{*}{$\begin{array}{c}\text { Kom- } \\
\text { plikationen }\end{array}$} & \multicolumn{2}{|c|}{ Lungenbefund } \\
\hline & & & & Eintritt & Entlassung \\
\hline 72 & $\begin{array}{l}\text { K., } \\
26 \mathrm{~J}_{.,} \\
\text {Erau }\end{array}$ & $\begin{array}{l}\text { Seit } 6 \text { Jahren bäufige } \\
\text { Bronchialkatarrhe } \\
\text { nach Influenza u. L } \\
\text { Lunge krank befund. } \\
\text { Vor } 3 \text { Jahr. Pleuritis } \\
\text { sicca, vor } 1 \text { 1/2 Jahr. } \\
\text { zieml. st. Hämoptoe, } \\
\text { die sich öfter wieder- } \\
\text { holte. T.-B. zahlreich } \\
\text { nachgewiesen. Seit } \\
6 \text { Monaten in einem } \\
\text { Davoser Sanatorium. } \\
\text { (Tuberkulink.Denys) }\end{array}$ & - & $\begin{array}{l}\text { L. Sp. H. Knacken; } \\
\text { R. Spitze raubes } \\
\text { Atmen. }\end{array}$ & $\begin{array}{l}\text { L. Spitze Knacken u. } \\
\text { ves.-bronch. Atmen. } \\
\text { Sonst reines Vesic.- } \\
\text { Atmen überall } \\
\end{array}$ \\
\hline 73 & $\begin{array}{l}\text { A., } \\
21 \text { J., } \\
\text { Lehrer }\end{array}$ & $\begin{array}{l}\text { Vor } 11 \text { Mon. rechts- } \\
\text { seitige Brustfellent- } \\
\text { zündung; seit } 2 \text { Mon. } \\
\text { wegen Lungenleiden } \\
\text { in Behandlung. } \\
\text { Stets fiebernd }\end{array}$ & - & $\begin{array}{l}\text { R. O.-Lappen relat. } \\
\text { Dämpf., Rauschen } \\
\text { beiderseits über den } \\
\text { Lungen. - Uber der } \\
\text { R. Spitze Exsp. ver- } \\
\text { längert u. Raubigk. }\end{array}$ & $\begin{array}{c}\text { Keine Raubigkeiten } \\
\text { mehr. - Rauschen } \\
\text { weniger ausge- } \\
\text { sprochen }\end{array}$ \\
\hline 74 & $\begin{array}{l}\text { H.', } \\
30 \text { J." } \\
\text { Eri. }\end{array}$ & $\begin{array}{l}\text { Krank seit } 5 / \pm \text { Jabr. } \\
\text { Beginn mit Pneu- } \\
\text { monie u. Bronchial. } \\
\text { katarrh, seitdem } \\
\text { Fieber, Husten, Aus- } \\
\text { wurf, Gewichtsabn. }\end{array}$ & - & $\begin{array}{l}\text { L. O. Lapp. schleppt. } \\
\text { rel. Dämpf. L. O.- } \\
\text { Lappen ves.-bronch. } \\
\text { u. Knattern vorn u. } \\
\text { hinten. R. Sp. rauhes } \\
\text { Atmen, rel. Dämpf. }\end{array}$ & $\begin{array}{l}\text { L. O.-L. rel. Dämpf. } \\
\text { dureh pur wenig } \\
\text { Knacken verdecktes } \\
\text { ves.bronch. Atmen } \\
\text { vornu. hinten. R.Sp. } \\
\text { rel. Dämpf., rauhes } \\
\text { ves. Atmen }\end{array}$ \\
\hline 75 & $\begin{array}{l}\text { J.", } \\
\text { Frl., }\end{array}$ & $\begin{array}{c}\text { Krank seit } 8 \text { Mon. } \\
\text { Beginn mit Husten, } \\
\text { Fieber. Appetit } \\
\text { schlecht }\end{array}$ & -- & $\begin{array}{l}\text { L. O.-Lappen relat. } \\
\text { Dämpf. ves.-bronch. } \\
\text { Atmen zwisthen } 2 \text {. } \\
\text { u. 3. Kippe vereinz. } \\
\text { Rhonchi, Atmen rauh } \\
\text { v.-bronch. b. bronch. } \\
\text { R. Sp. rel. Dämpf. }\end{array}$ & $\begin{array}{l}\text { L. Sp. rel. Dämpf. } \\
\text { L. O.L. ves.-bronch. } \\
\text { Atmen, zwischen } 2 . \\
\text { a. 3. Rippe vereinz. } \\
\text { Rhonchi. R. Sp. rel. } \\
\text { Dämpf., keine Rb. }\end{array}$ \\
\hline 76 & $\begin{array}{l}\text { K., } \\
22 \mathrm{~J} ., \\
\text { Frl. }\end{array}$ & $\begin{array}{l}\text { Krank seit }{ }^{3} \text { Jahr. } \\
\text { T.-B. vor } 4 \text { Mon. } \\
\text { nachgewies. Husten, } \\
\text { Fieber. Ferschlimm. } \\
\text { durch Influenza vor } \\
3 \text { Wochen. Schlaf u. } \\
\text { Appetit schlecht, } \\
\text { Dyspnoe beim Berg- } \\
\text { steigen }\end{array}$ & - & $\begin{array}{l}\text { R. Sp. rel. Dämpf. } \\
\text { ves.-bronch. Atmen } \\
\text { (Narbe). L. O.-Lapp. } \\
\text { rel. Dämpf. Spitze } \\
\text { ves.-bronch. amphor. } \\
\text { Atmen, gloss. halb. } \\
\text { klingendes Rasseln, } \\
\text { hinten idem, ves.- } \\
\text { bronch. Knattern. } \\
\text { Über beidenU.-Lapp. } \\
\text { Rauschen }\end{array}$ & $\begin{array}{l}\text { R. Sp. unveränderl. } \\
\text { Retraktion des L. } \\
\text { O.-Lapp. rel. Dämpf. } \\
\text { ves.-bronch. Atmen } \\
\text { mit zähem Knarren } \\
\text { und Knattern }\end{array}$ \\
\hline 77 & $\begin{array}{l}\text { L., } \\
30 \text { J., } \\
\text { Arzt }\end{array}$ & $\begin{array}{l}\text { Seit längerel Zeit } \\
\text { heiser. Mehrere Mon. } \\
\text { in einem Davoser } \\
\text { Sanat. ohne Erfulg, } \\
\text { T.-B. nachgewiesen }\end{array}$ & $\begin{array}{l}\text { Larynx. Heiser- } \\
\text { keit. Stimm- } \\
\text { bänder gerötet, } \\
\text { Hinterwand } \\
\text { verdiekt. } \\
\text { Entlas a n } \mathrm{g:} \\
\text { Kehlkopf frei, } \\
\text { keine Heiser- } \\
\text { keit }\end{array}$ & $\begin{array}{l}\text { Über beiden O.-Lapp. } \\
\text { relat. Dämpf. ves.: } \\
\text { bronch. Atmen, R. } \\
\text { O.-Lappen Knacken }\end{array}$ & $\begin{array}{l}\text { Über beiden Spitzen } \\
\text { leises vesik.-bronch. } \\
\text { Atmen. R.hint.rauh. } \\
\text { Keine Rhonchi }\end{array}$ \\
\hline
\end{tabular}




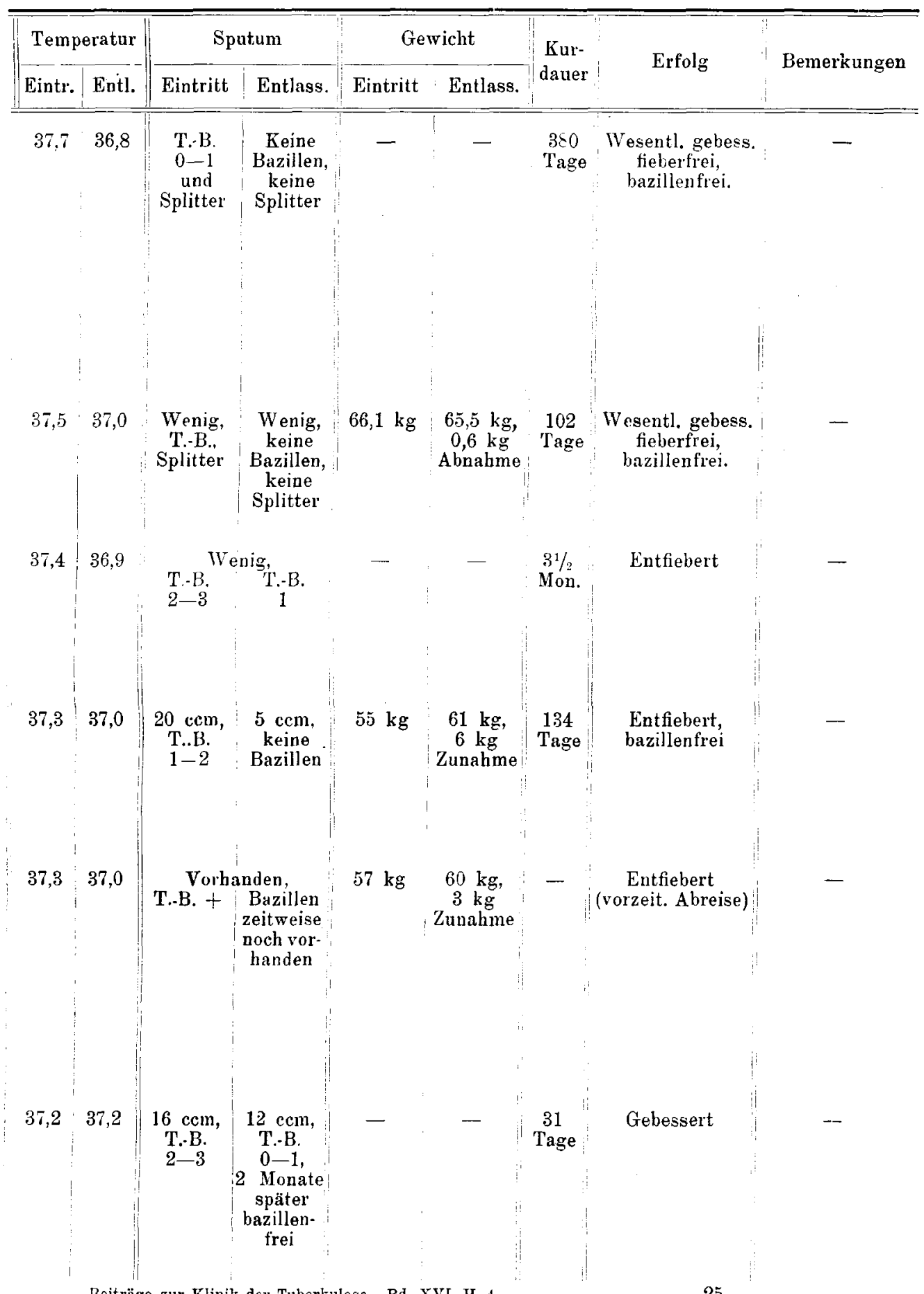




\begin{tabular}{|c|c|c|c|c|c|}
\hline \multirow{2}{*}{$\mathrm{Nr}$} & \multirow{2}{*}{$\begin{array}{l}\text { Name, } \\
\text { Alter, } \\
\text { Beruf }\end{array}$} & \multirow{2}{*}{ Anamnese } & \multirow{2}{*}{$\begin{array}{c}\text { Kom- } \\
\text { plikationen }\end{array}$} & \multicolumn{2}{|c|}{ Lungenbefund } \\
\hline & & & & Eintritt & Entlassung \\
\hline 78 & $\begin{array}{l}\text { M., } \\
\text { Kaufmann }\end{array}$ & $\begin{array}{l}\text { Vater wahrscheinl. } \\
\text { tuberkulös. Vor } 14 \\
\text { Tagen durch zufäll. } \\
\text { Untersuchung } \mathrm{T} . \mathrm{B} \text {. } \\
\text { festgestellt. Etwas } \\
\text { Husten }\end{array}$ & - & $\begin{array}{l}\text { Rauschen über beid. } \\
\text { Lungen. Leises, un- } \\
\text { vollkommen. Atmen, } \\
\text { schlechte Expansion. } \\
\text { L. Spitze rel. Dämpf. } \\
\text { Unterbalb der Spina } \\
\text { scapulae an einer } \\
\text { Stelle fein. Knacken }\end{array}$ & $\begin{array}{c}\text { Atmen über den } \\
\text { Spitzen abgeschw., } \\
\text { rauschend. Keine } \\
\text { Rhonchi }\end{array}$ \\
\hline 79 & $\begin{array}{l}\text { R., } \\
33 \text { J., } \\
\text { Pfarrer }\end{array}$ & $\begin{array}{l}\text { Mutter an Tbc. } \uparrow \text {. } \\
\text { Krank seit } 2 \text { Mon. } \\
\text { Wenig Sputum } \\
\text { morgens. } \\
\text { T.-B. vorhanden! } \\
\text { Iemperatur erhöht }\end{array}$ & - & $\begin{array}{l}\text { Beide Sp. rel. Dämpf. } \\
\text { ves.bronch. Atmen } \\
\text { mit st. verl. Exspir. } \\
\text { L. mehr wie R. } \\
\text { Hinten oben beiders. } \\
\text { Rauhigkeiten, links } \\
\text { etwas Knacken. } \\
\text { Intersk. Rauschen }\end{array}$ & $\begin{array}{l}\text { R. Sp. rel. Dämpf. } \\
\text { Abgeschw. vesic.- } \\
\text { bronch. Atmen ohne } \\
\text { Rhonchi }\end{array}$ \\
\hline 80 & $\begin{array}{l}\text { A., } \\
\text { Offizier }\end{array}$ & $\begin{array}{l}\text { Seit } 2 \text { Jahren krank. } \\
2 \text { mal in Arosa. } \\
\text { Vor } 1 / 2 \text { J. Influenza. } \\
\text { Fieber, Husten. Erbl. } \\
\text { Belastung }\end{array}$ & - & $\begin{array}{l}\text { R. H. O.-Lappen } \\
\text { Knattern (mittleres) } \\
\text { U.-L. V. und LHO. } \\
\text { rauhes Atmen. LLH. } \\
\text { seitl. ahgeschwächt. } \\
\text { rauhes Atmen }\end{array}$ & Kein Knattern \\
\hline 81 & $\begin{array}{l}\text { B. } \\
\text { Student }\end{array}$ & $\begin{array}{l}\text { Erbliche Belastung. } \\
\text { Vor } 3 \text { u. } 2 \text { Jahren } \\
\text { Hämoptoe; Husten, } \\
\text { Müdigkeit, Fieber }\end{array}$ & - & $\begin{array}{l}\text { L O.-L. u. R. Sp. rel. } \\
\text { Dampf. ves.-bronch. } \\
\text { Atmen. Knattern } \\
\text { (mittel u. fein) }\end{array}$ & $\begin{array}{l}\text { L O-L. ves.-bronch. } \\
\text { 3. bis 4. Costa Er- } \\
\text { offnungsknattern. R. } \\
\text { Spitze ves.-bronch. }\end{array}$ \\
\hline 82 & $\begin{array}{l}\text { D., } \\
25 \mathrm{~J}_{.}, \\
\text {Erl. }\end{array}$ & $\begin{array}{c}\text { Seit } 3 \text { Mon. Husten, } \\
\text { Nachtschweiss, } \\
\text { leichte Temperatur- } \\
\text { erhöhung }\end{array}$ & 一 & $\begin{array}{l}\text { Rel. Dämpf. über } \\
\text { beid. O. Lappen, ab. } \\
\text { geschw. Atmen das, } \\
\text { rauh. Rauschen über } \\
\text { beiden Lungen }\end{array}$ & $\begin{array}{l}\text { Spitzenatmen ver- } \\
\text { schärft. Exspir. ver- } \\
\text { läng. } 2 \text { '. noch rauh }\end{array}$ \\
\hline 83 & $\begin{array}{c}\text { V., } \\
45 \stackrel{J}{.}, \\
\text { Professor }\end{array}$ & $\begin{array}{l}\text { Erbliche Belastung. } \\
\text { Seit } 3 \text { Jahren Husten } \\
\text { u. Auswurf, } 3 \text { mal } \\
\text { Hämoptoe }\end{array}$ & - & $\begin{array}{l}\text { R Seite schleppt. ROL } \\
\text { Dampf, voln bis 3., hint. } \\
\text { bis 5. Costa, brorcl. } \\
\text { Atmen, verläng. Exspil. } \\
\text { feine Rhonchi. L. bis } \\
\text { 2. Costa u. " }{ }^{2} \text { Scapulae } \\
\text { Sehallkürz, knatternde } \\
\text { Rhonchi. HU. sackadier. } \\
\text { Atmen. Rausclien }\end{array}$ & $\begin{array}{l}\text { R. O.-L rel. Dämpf. } \\
\text { Ohne Rhonchi. L. } \\
\text { Sp. ves.-bronch. rauh }\end{array}$ \\
\hline 84 & $\begin{array}{l}\text { A., } \\
17 \text { J., } \\
\text { Student }\end{array}$ & $\begin{array}{l}\text { Vor } 4 \text { Jahren Pleurit. } \\
\text { sicca dextra. Inner- } \\
\text { halbJahresfrist } 2 \text { mal } \\
\text { Influenza, seit dem } \\
\text { letzt. Anfall Husten, } \\
\text { Auswurf, Müdigkeit }\end{array}$ & - & $\begin{array}{l}\text { R. Sp. vornu. hinten } \\
\text { Schallkürzung, ver- } \\
\text { schärft. Inspir, ver- } \\
\text { längert. Exspirium, } \\
\text { bronch.halbtrockene } \\
\text { Rhonchi. KHU. von } \\
\text { Angulus Scap. ab- } \\
\text { wärts Schwarte. } \\
\text { Rauschen beiders. }\end{array}$ & $\begin{array}{l}\text { R. Sp. rel. Dämpf. } \\
\text { Exspir. verl. ves.- } \\
\text { bronch. Atmen. } \\
\text { RHU. vereinzelte, } \\
\text { feinste knatt. Rh. } \\
\text { Schwarte. L. Spitze } \\
\text { hinten Rauschen }\end{array}$ \\
\hline
\end{tabular}




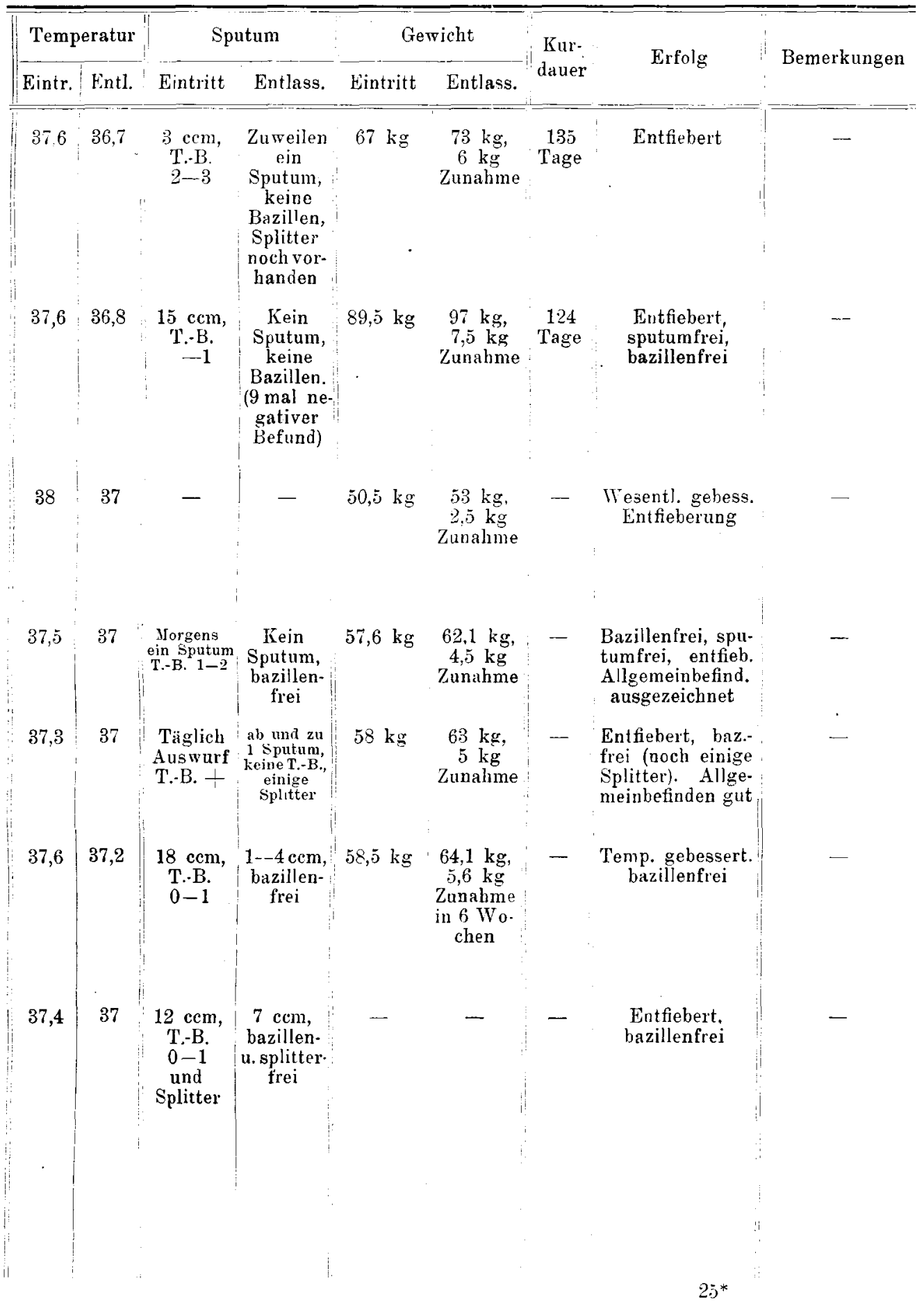


Klasse II.

\begin{tabular}{|c|c|c|c|c|c|}
\hline \multirow{2}{*}{ Nr. } & \multirow{2}{*}{$\begin{array}{l}\text { Name, } \\
\text { Alter, } \\
\text { Beruf }\end{array}$} & \multirow{2}{*}{ Anamnese } & \multirow{2}{*}{$\begin{array}{c}\text { Kom- } \\
\text { plikationen }\end{array}$} & \multicolumn{2}{|c|}{ Lungenbefund } \\
\hline & & & & Eintritt & Entlassung \\
\hline 85 & $\begin{array}{l}\text { R., } \\
\text { 31 J., } \\
\text { Katufmann }\end{array}$ & $\begin{array}{l}\text { Seit } 5 \text { Jahren krank, } \\
1^{1 / 2} \text { Jahre in Arosa }\end{array}$ & - & $\begin{array}{l}\text { R. Lunge schleppt; } \\
\text { vesik.-bronch. bis } \\
\text { bronch.- amphor. u. } \\
\text { Knattern über der } \\
\text { ganzen Lunge. L. Sp. } \\
\text { ves.-bronch.-bronch. }\end{array}$ & $\begin{array}{l}\text { R O.-Lapp. zerstreut } \\
\text { Knattern, bes. nach } \\
\text { Husten; M.-Lappen } \\
\text { frei,U.Lapp, welche, } \\
\text { feine, spärliche Ge } \\
\text { räusche - RHU. } \\
\text { etwas Reiben. L. } \\
\text { keine Rhonchi }\end{array}$ \\
\hline 86 & $\begin{array}{l}\text { C., } \\
34 \text { J., } \\
\text { Kapitän }\end{array}$ & $\begin{array}{l}\text { Seit einigen Monaten } \\
\text { erfolglos in Davos }\end{array}$ & - & $\begin{array}{l}\text { R. O.-L. ves.-bronch. } \\
\text { Atmen, feines und } \\
\text { mittl. Knatitern. } \\
\text { Rel. Dämpf. L. Sp. } \\
\text { ves.bronch.u.Knatt. } \\
\text { Rel. Dämpf. }\end{array}$ & $\begin{array}{l}\text { R. Sp. Exsp. ves.- } \\
\text { bronch. I. Sp. ab- } \\
\text { geschw. ves. Atmen. } \\
\text { Nirg. Rhonchi. }\end{array}$ \\
\hline 87 & $\begin{array}{l}\text { T., } \\
\text { Techniker }\end{array}$ & $\begin{array}{l}\text { Vor } 16 \mathrm{Jahr} \text {. geringe } \\
\text { Hämoptoe. Vor } 5 \mathrm{~J} \text {. } \\
5 \text { Mon. Kur in einen } \\
\text { DavoserSauatorium. } \\
\text { In den letzten Mon. } \\
3 \text { mal Hämoptoe. } \\
\text { Morgens Auswurf }\end{array}$ & - & $\begin{array}{l}\text { Rauschen über beid. } \\
\text { Lungen. I. Spitze } \\
\text { Knattern. R. Spitze } \\
\text { ves.bronch. rauh }\end{array}$ & $\begin{array}{l}\text { Über den Spitzen } \\
\text { ves.-bronch. Atmen, } \\
\text { keine Rhunchi }\end{array}$ \\
\hline
\end{tabular}

P., Mutter ,lungenkr. 4 Nierentuberkul. $22 \mathrm{~J}$, Frl.

35 J., Baner

\begin{abstract}
Seit $3 \mathrm{~J}$ ahren wegen Im Urin Eiweiss Lungen- u. Nieren- Urinreakt. anf tuberkulose in Be- neutral, nach handl. Alle 'Tuber- einigen Injekt. kuline nicht ver. Zum Schluss der tragen, dageg. Besse- Behandlung $\mathrm{E}$ rung auf Einreibung. weiss meist mit I.-K. (laut Arzt. zeitweise nocla bericht.) - Zuzeit Spuren. Keine Nierenschmerzen Splitter.
\end{abstract}

Seit 6 Jahren krank. In letzter Zeit Fieber $38^{\circ}$, Sputum $60 \mathrm{ccm}$

Klasse II.

R. Spitze Narbenatmen
Wie bei der Auf. nahme
R. O.-Lappen hinten Knacken mit Klang, vorn vesik.-bronch. rauh. - Rel. Dämpf. - UL. scharf vesik. L. Lunge Rauschen, Spitze hinten vereinzeltes Knacken
Vorzeitige Abreise ohne Schlussunter suchung 
Stadium 2-3.

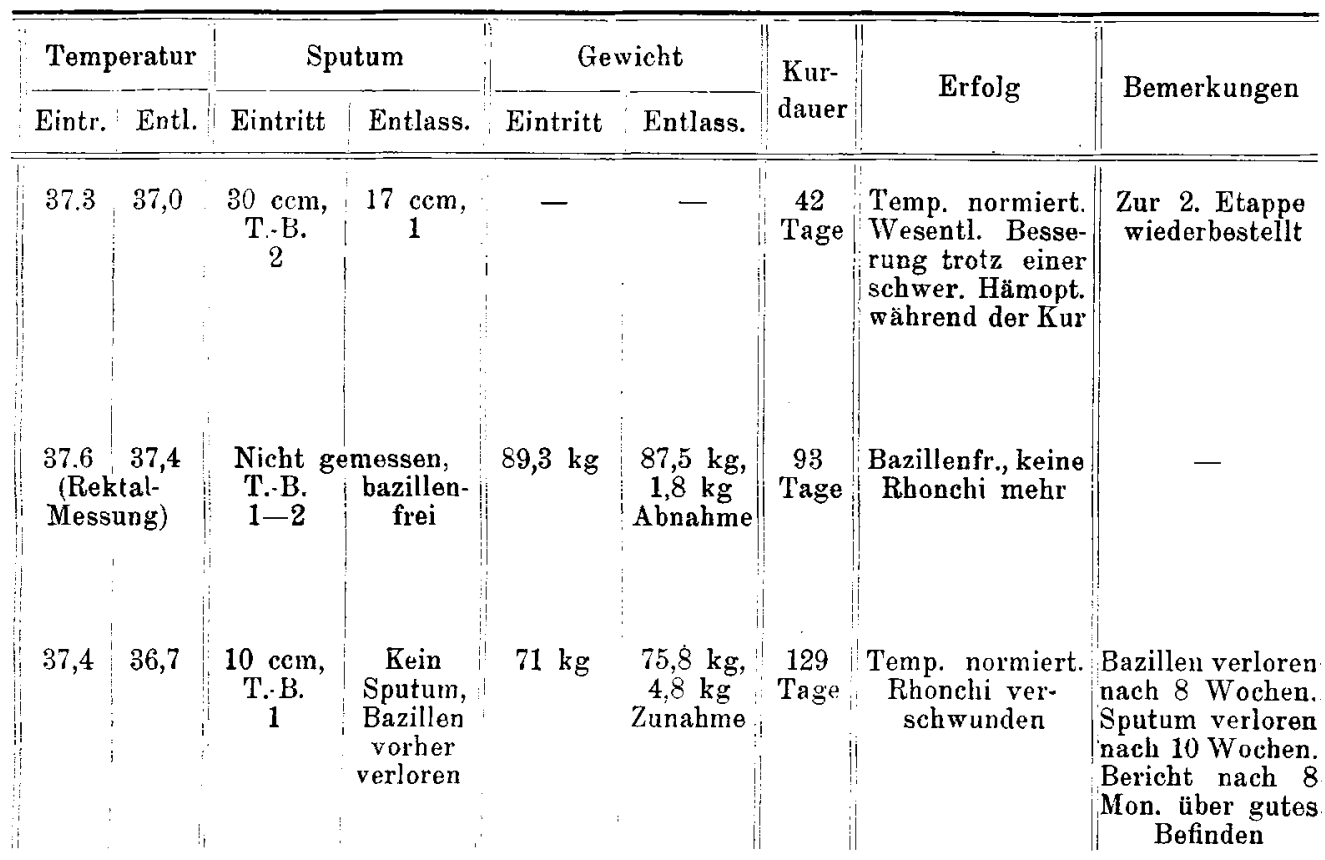

\section{Stadium 3.}

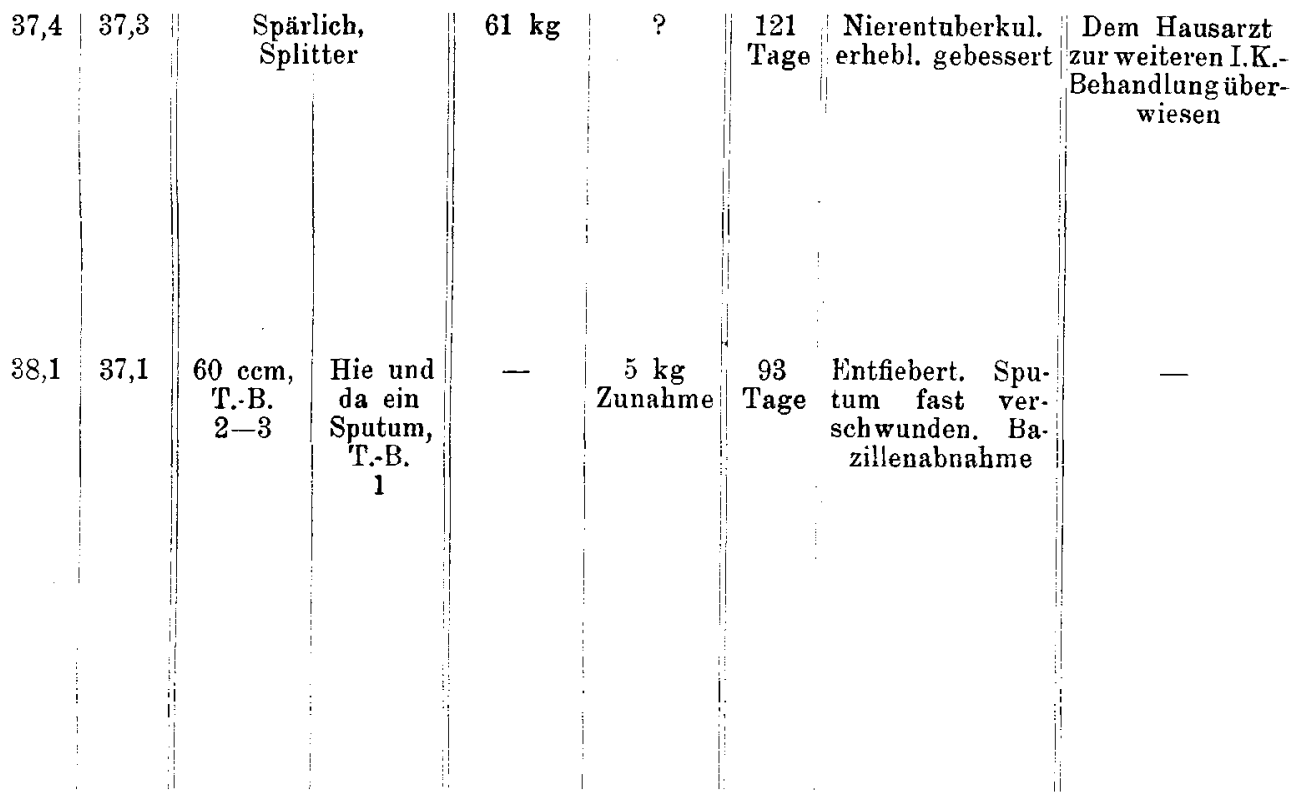




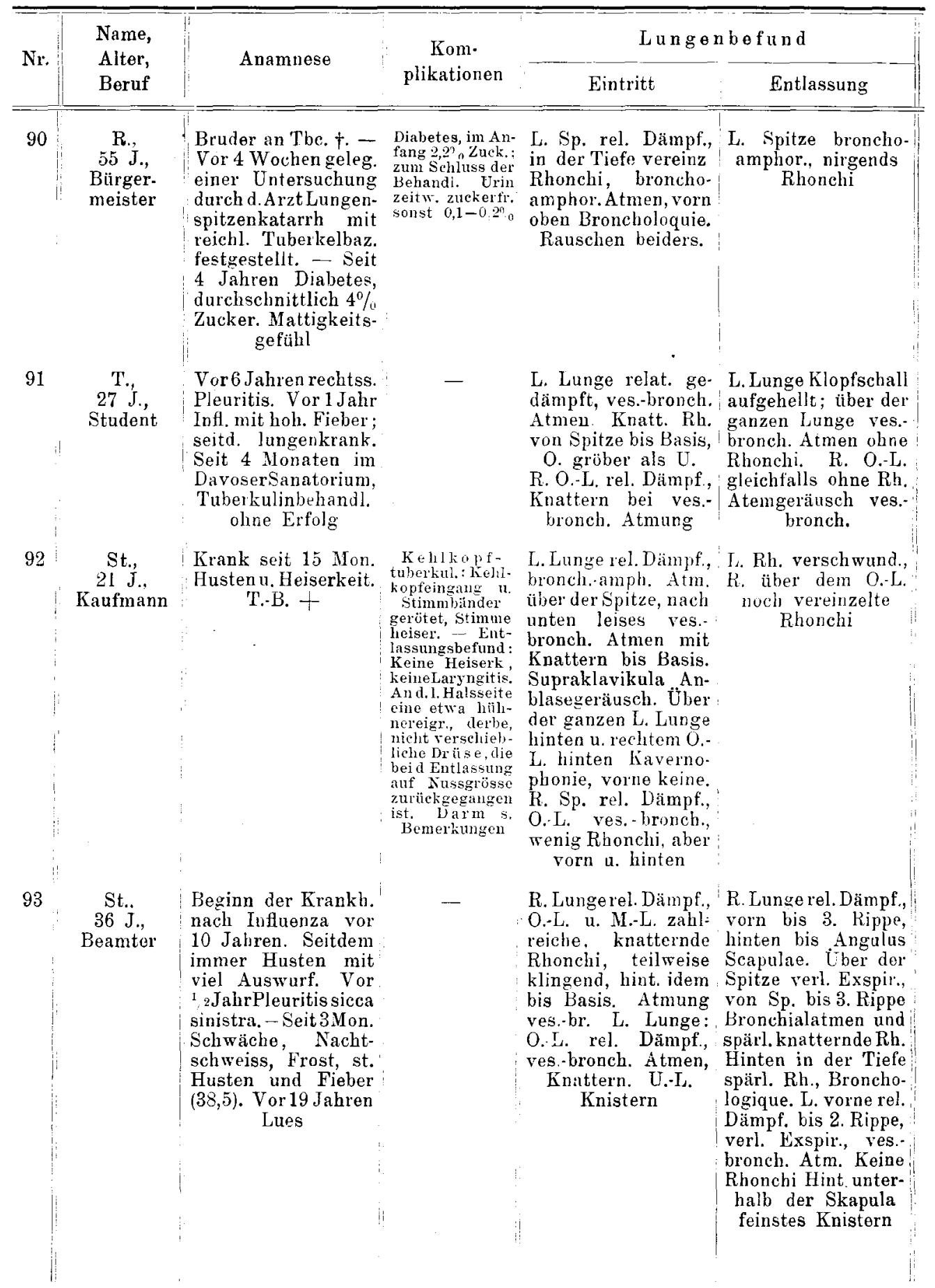




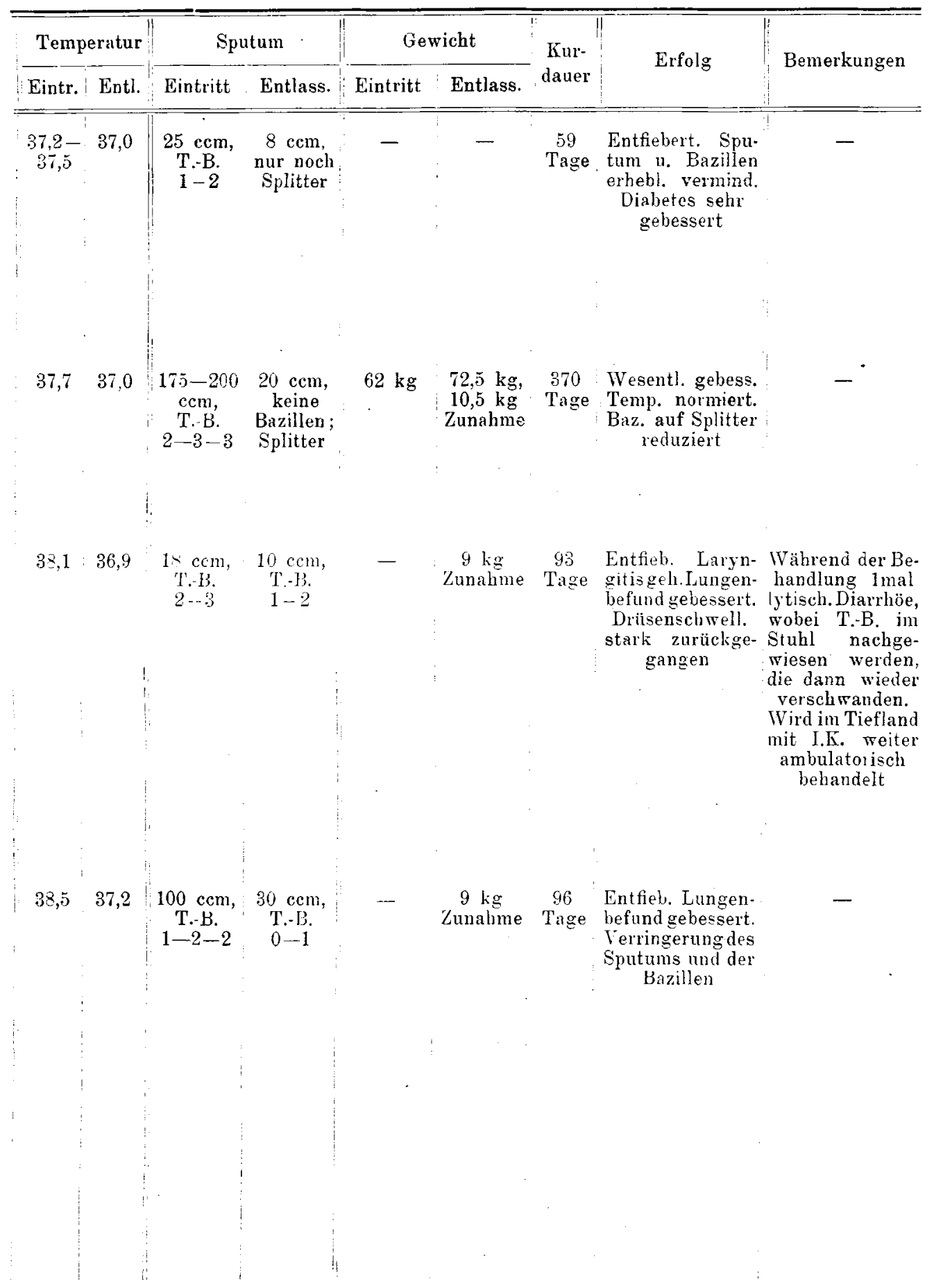




\begin{tabular}{|c|c|c|c|c|c|}
\hline \multirow{2}{*}{$\mathrm{Nr}$} & \multirow{2}{*}{$\begin{array}{l}\text { Name, } \\
\text { Alter, } \\
\text { Beruf }\end{array}$} & \multirow{2}{*}{ Anamnese } & \multirow{2}{*}{$\begin{array}{c}\text { Kom- } \\
\text { plikationen }\end{array}$} & \multicolumn{2}{|c|}{ Lungen befund } \\
\hline & & & & Eintritt & Entlassung \\
\hline 94 & $\begin{array}{l}\text { P., } \\
\text { Ërl. }\end{array}$ & $\begin{array}{l}\text { Vater an Tbc. †. - } \\
\text { Krank seit } 3 \text { Jahren, } \\
\text { zahlieiche Blutung., } \\
\text { besonders in letzter } \\
\text { Zeit. Sanatoriumsbe- } \\
\text { handl. ohne wesentl. } \\
\text { Erfolg. Vor } 2 \text { Mon. } \\
\text { Pleuritis, seit } 1 \text { Non. } \\
\text { in Davos ohne Fort- } \\
\text { schritte. Seit mehrer. } \\
\text { Jahr. Magen-Darn- } \\
\text { katarrh. Geringe } \\
\text { Leistungsfähigkeit. } \\
\text { Schlaf u. Appetit } \\
\text { schlecht }\end{array}$ & $\begin{array}{l}\text { Darmtuberkul. } \\
\text { Obstipation, } \\
\text { Krämpfe. Im } \\
\text { Stuhl Stäbehen } \\
\text { t. Splitter, bei } \\
\text { der Entlassung } \\
\text { nur nochSplitter }\end{array}$ & $\begin{array}{l}\text { L. Lunge rel. Dämpf., } \\
\text { abgeschwächt. ves.- } \\
\text { bronch. Atmen über } \\
\text { dem O.-L. u. U.-L., } \\
\text { rauhes Atmen. Zer- } \\
\text { streute knackende } \\
\text { Rhonchi, über der } \\
\text { Spitze metallisches } \\
\text { Knacken. R. Spitze } \\
\text { ves.-bronch. u. rauh }\end{array}$ & $\begin{array}{l}\text { L. Lunge atmet gut, } \\
\text { Spitze ves.-bronch. } \\
\text { Atm. ohne Rhonchi. } \\
\text { O.-L. ves. - bronch., } \\
\text { U.-L. scharf vesik. } \\
\text { Atmen. R. ves.-br. } \\
\text { Atmen, obne Rh. u. } \\
\text { Rauhigkeiten }\end{array}$ \\
\hline 95 & $\begin{array}{c}\text { R.., } \\
34 \text { J., } \\
\text { Erau }\end{array}$ & $\begin{array}{l}\text { Ein Bruder lungenkr. } \\
\text { Beginn vor } 2 \text { Jahren } \\
\text { mit Husten u. Aus- } \\
\text { wurf, leicht. Fieber, } \\
\text { Nachtschweissen, } \\
\text { Mattigkeit, Appetit- } \\
\text { losigkeit. - Klimat. } \\
\text { Kuren ohne Erfolg. } \\
\text { - Vor } 1 / 2 \text { Jahr I.K.- } \\
\text { Behandl., die wesent- } \\
\text { liche Besserung } \\
\text { brachte, aber vor- } \\
\text { zeitig abgebrochen } \\
\text { wurde }\end{array}$ & - & $\begin{array}{l}\text { R. O.L. gedämpft } \\
\text { tympanit., bronch.- } \\
\text { amph. Atmen, grosse } \\
\text { n. mittl. klingende } \\
\text { Rhonchi u. Knarren. } \\
\text { L. Sp. ves.-bronch., } \\
\text { rauh }\end{array}$ & $\begin{array}{l}\text { R. Spitze V. u. H. } \\
\text { bronch. amplior., ab } \\
\text { und zu Knacken, } \\
\text { Kavernoloquie. Nirg. } \\
\text { Rhonchi mehr }\end{array}$ \\
\hline 96 & $\begin{array}{c}\text { K., } \\
37 \text { J., } \\
\text { Ingenieur }\end{array}$ & $\begin{array}{l}\text { Beginn der Krankh. } \\
\text { vor } 1^{1} \text { Jahren mit } \\
\text { Mattigkeit. Später } \\
\text { Husten, Appetit- } \\
\text { losigk., Schlaflosig. } \\
\text { keit. In letzter Zeit } \\
\text { immer fiebernd }\end{array}$ & - & $\begin{array}{l}\text { R. O.-L. rel. tymp. } \\
\text { Dämpf., ves.-bronch.- } \\
\text { amph. Atmen. V. } \\
\text { mittl.u. grossekling. } \\
\text { Rhonchi bis 5. Rippe, } \\
\text { seitlich idem. - H. } \\
\text { ves.-bronch. Atmen } \\
\text { u. Knattern. L. Lunge } \\
\text { ves.-bronch.Atmung. } \\
\text { Rhonchi in geringer } \\
\text { Zahl von Spitze bis } \\
\text { Basis, daneb.Giemen } \\
\text { u. Pfeifen }\end{array}$ & $\begin{array}{l}\text { Über beiden O.-L. } \\
\text { ves..broncho.bronch. } \\
\text { Atmen u. über der } \\
\text { r. Spitze mit amph. } \\
\text { Beiklang. Ganz selt. } \\
\text { Rhonchi }\end{array}$ \\
\hline is & & ! & & u. Premen & \\
\hline 97 & $\begin{array}{c}\text { W., } \\
40 \stackrel{\mathrm{J} .,}{ } \\
\text { Kaufmann }\end{array}$ & $\begin{array}{l}\text { Vor } 1^{1} \text { Jahr. Husten, } \\
\text { vor } 1 \text { Jahr mehrfach } \\
\text { Hämoptoe mit nach. } \\
\text { folgendem Fieber. } \\
1 / 2 \text { Jahr in einem } \\
\text { Sanatorium in Eng- } \\
\text { land. Im Sputum T.- } \\
\text { B. vorhanden. Temp.- } \\
\text { Erhöhung }\end{array}$ & - & $\begin{array}{l}\text { R. O. Lapp. schleppt } \\
\text { stark, rel. Dämpf. } \\
\text { Hinten u.vornKnatt. } \\
\text { In der Spitze eine } \\
\text { Kaverne mit Rh. } \\
\text { L. Spitze rauh, zu. } \\
\text { scharf }\end{array}$ & $\begin{array}{l}\text { Über der R. Spitze } \\
\text { hinten nach Husten } \\
\text { vereinz. Knacken. In } \\
\text { der Spitze Kaverno- } \\
\text { loquie, keineRhonchi }\end{array}$ \\
\hline
\end{tabular}




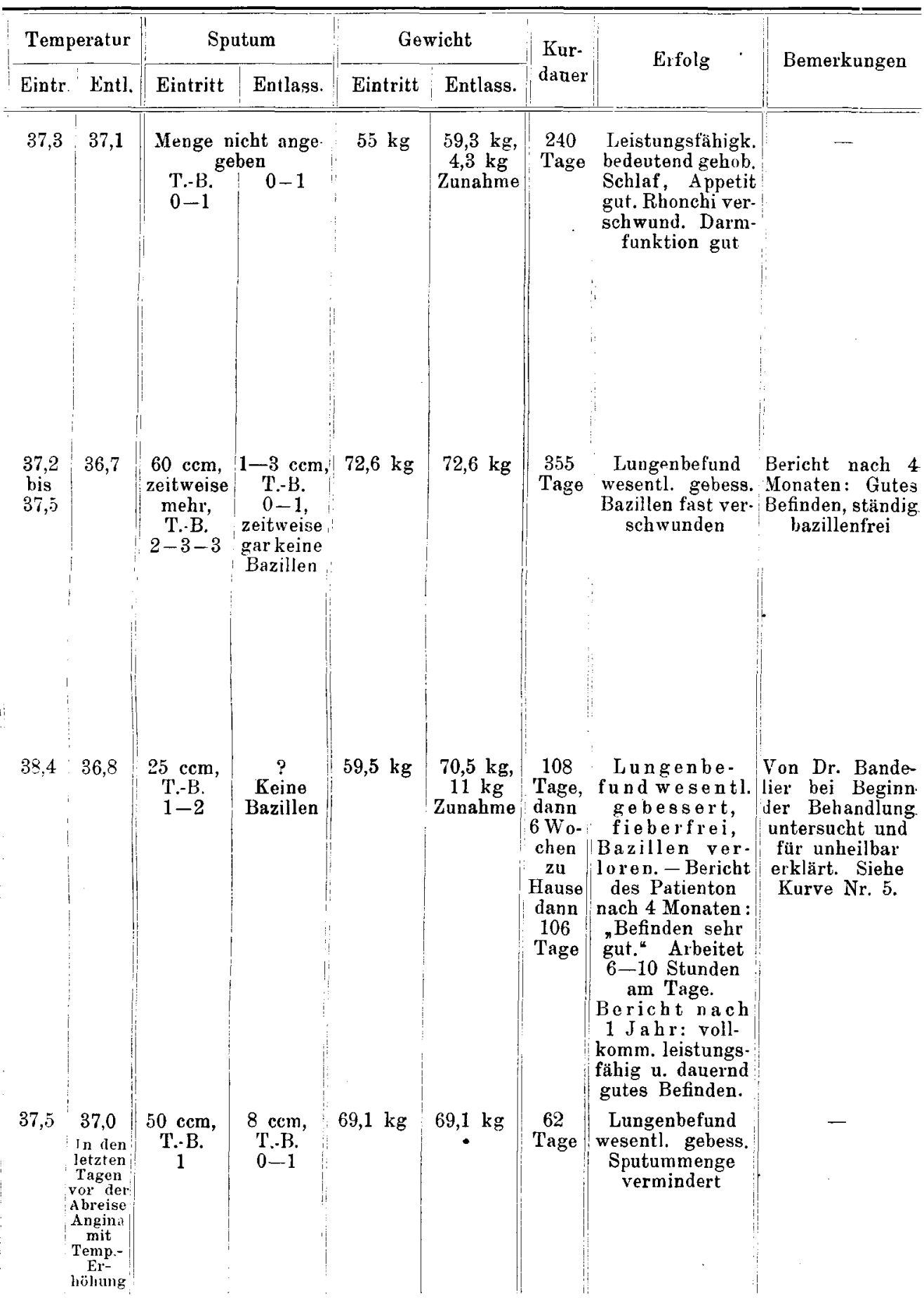




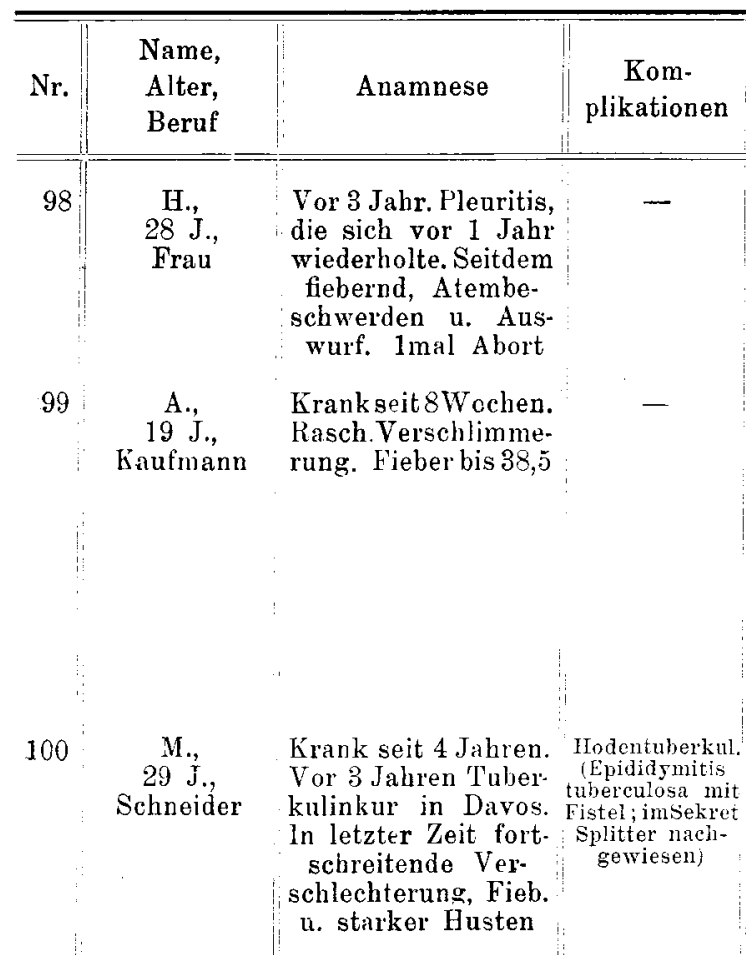

20 . J., Student
Beginn der Krankh. vor 8 Monaten mit schwererLungenentzündung. - Auch später wochen], hoch fiebernd (über $39^{\circ}$ ), bettlägerig. Tuberkulose diagnostiz. Schlechtes Befinden nach jeder Richtung hin

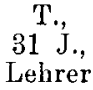

Vater tuberkulös.

Krank seit 6 Monat. Husten, Auswurf, Nachtschweisse. Mehrfach Blutung. Vorbehandlung mit I.K. mit gutem Erfolg
RH. relat. Dämpf., R. O.L. ves.-bronch, rauhes, abgeschw. keine Rhonchi. U.-I. Atmen, über dem U.- : keine Rhonchi, keine L. Rhonchi. L. Sp. Rauhigkeiten. L. Sp. rel. Dämpf. rel. Dämpf.

L. O.-L. rel. Dämpf. L. O.-L. starke rel. ves.-bronch. Atmen. Dämpf. mit Spur Knarren und grobe Tympanie, bronchohalbkling. Rhonchi. amph. Atmen, keine R. Spitze rel.Dämpf. Rhonchi, nur in der vornves.-bronch. mit Spitze etwas Knack. Rauhigkeiten, hinten u. Knarren. L O.-L. feineklonchi. In den Atmung abgeschw., ersten Tagen der Kur rauh, ves.-bronch. Pleuritis exsudativa R. Sp. ves.-bronch. $\sin$.

R. O. Lapp. Dämpf., R. Sp. rel. Dämpf. branch-amph. Atm.. ves.-bronch. Atmen, hinten bronchial. - Kavernoloquie, Vorn u. hinten im Be- keine Rhonchi. reich des O.-Lüberall Infraklavik, Eröttknatt.Rhonchi, in der nungsknistern. InterSpitze klingend. L. scap. einzelne feinste Spitze rel. Dämpf., Rhoncbi. L. Lunge Exsp, verlängert $u$. reines ves, Atmen, verschärft hinten etwas versch.

L. "Pottenger", über Rel. Dämpf. über O.-L. tymp. Dämpf. beiden O.L. L. mit bis zur halben Höhe etwas Tympanie. L. der Lunge. - Über Sp. Kavernoloquie u. dem ganzen O.-L.- Knattern bis3.Rippe. mittelgross-blasige, $R$. keine Rhonchi halbkling. Rasselge- U.-L. beiders, abgeräusche. R. O.Lapp. schwächtes Atmen. Dämpf., feine Rh. u. Rauschen geringer halbkling. Rasseln. Uberal! beiders. abgeschw. rauhes und rauschendes Atmen

LHO.-Angulus sich LHU.rauhes Atmen, verlier. Schallkürz. nirgends Rhonchi. Intersc. ves,-bronch. VR. infraklavikular Atmen u. Kuattern verschärftes vesik, Atmen 


\begin{tabular}{|c|c|c|c|c|c|c|c|c|}
\hline \multicolumn{2}{|c|}{ Temperatur } & \multicolumn{2}{|c|}{ Sputum } & \multicolumn{2}{|c|}{ Gewicht } & \multirow{2}{*}{$\begin{array}{l}\text { Kur. } \\
\text { dauer }\end{array}$} & \multirow{2}{*}{ Erfolg } & \multirow{2}{*}{ Bemerkungen } \\
\hline Eintr. & Entl. & Eintritt & Entlass. & Eintritt & Entlass. & & & \\
\hline 33,1 & 37,2 & $\begin{array}{l}? \\
\text { T.-B. } \\
2-3\end{array}$ & $\begin{array}{l}\text { Kein } \\
\text { Sputum, } \\
\text { keine } \\
\text { Bazillen, } \\
\text { keine } \\
\text { Splitter }\end{array}$ & $62.5 \mathrm{~kg}$ & $\begin{array}{c}63,5 \mathrm{~kg} \\
1 \mathrm{~kg} \\
\text { Zunahme }\end{array}$ & $\begin{array}{l}77 \\
\text { Tage }\end{array}$ & $\begin{array}{l}\text { Lungenbefund } \\
\text { wesentl. gebess. } \\
\text { Sputum verloren. } \\
\text { Bazillen verlor. } \\
\text { Keine Atembe. } \\
\text { schwerden mehr }\end{array}$ & - \\
\hline $\begin{array}{l}39,0 \\
\text { und } \\
\text { mehr }\end{array}$ & 37.1 & $\begin{array}{c}45 \mathrm{ccm} \\
\text { T'-B. } \\
2-3\end{array}$ & $\begin{array}{c}10 \mathrm{ccm} \\
\text { T. } \mathrm{B} \\
1-2\end{array}$ & $68 \mathrm{~kg}$ & $\begin{array}{c}66 \mathrm{~kg} \\
2 \mathrm{~kg} \\
\text { Abnahme } \\
\text { (durch } \\
\text { protra- } \\
\text { hierte Re } \\
\text { aktionen } \\
\text { erklärt) }\end{array}$ & $\begin{array}{l}275 \\
\text { Tage }\end{array}$ & $\begin{array}{c}\text { Temp. normiert. } \\
\text { Lungenbefund } \\
\text { wesentl. gebess. } \\
\text { Starke Sputum- } \\
\text { abnahme }\end{array}$ & $\begin{array}{l}\text { Siehe Kurve } \\
\text { Nr. } 6\end{array}$ \\
\hline 37,9 & 37,2 & $\begin{array}{c}60 \text { ccm, } \\
\text { T.-B. } \\
1-2-2\end{array}$ & $\begin{array}{c}\text { Kein } \\
\text { Sputum, } \\
\text { beine } \\
\text { Bazillen, } \\
\text { mir nocli } \\
\text { splitter ror } \\
\text { Verschwin- } \\
\text { den des } \\
\text { Sputums }\end{array}$ & $63 \mathrm{~kg}$ & $\begin{array}{c}74 \mathrm{~kg}, \\
11 \mathrm{~kg} \\
\text { Zunahme }\end{array}$ & $\begin{array}{l}280 \\
\text { T'age }\end{array}$ & $\begin{array}{l}\text { Lungenbefund } \\
\text { wesentl. gebess. }\end{array}$ & $\begin{array}{l}\text { Im Verlauf der } \\
\text { Krankheit tritt } \\
\text { eineEpididymitis } \\
\text { auf, die ohne ope- } \\
\text { rative Behandl. } \\
\text { in kurz.Zeitheilt. } \\
\text { (Tom Chirurgen } \\
\text { wurde sofortige } \\
\text { Exstirpation ge- } \\
\text { raten.) Noch in } \\
\text { Behandlung }\end{array}$ \\
\hline
\end{tabular}

$39,2 \quad 36,860 \mathrm{ccm}, \quad 6 \mathrm{~cm}, \quad 57,5 \mathrm{~kg} \quad 65 \mathrm{~kg}, \quad 95$ Fieberfr.Lungen. Noch in BehandT.B. T. $\quad$ T. B $2-2-3 \quad 1-2$

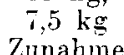

Zunahme
Tage befund wesentl. lung. S. Kurve gebess. Sputum- Nr. 4 menge erheblich reduziert

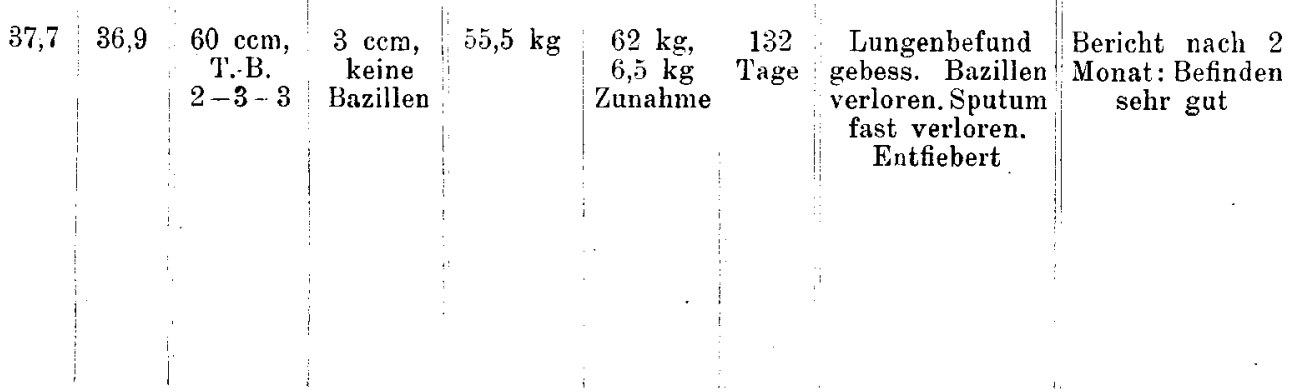




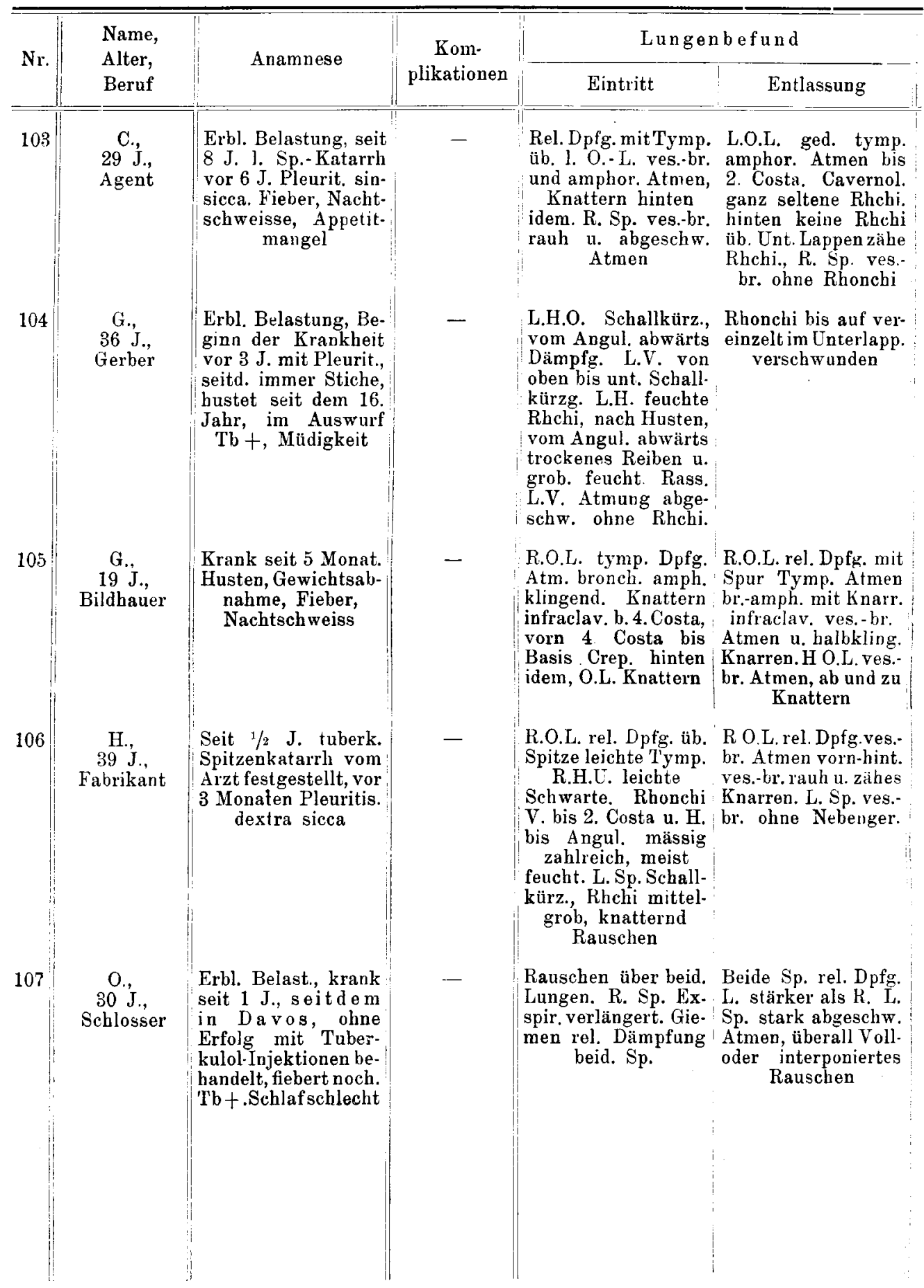




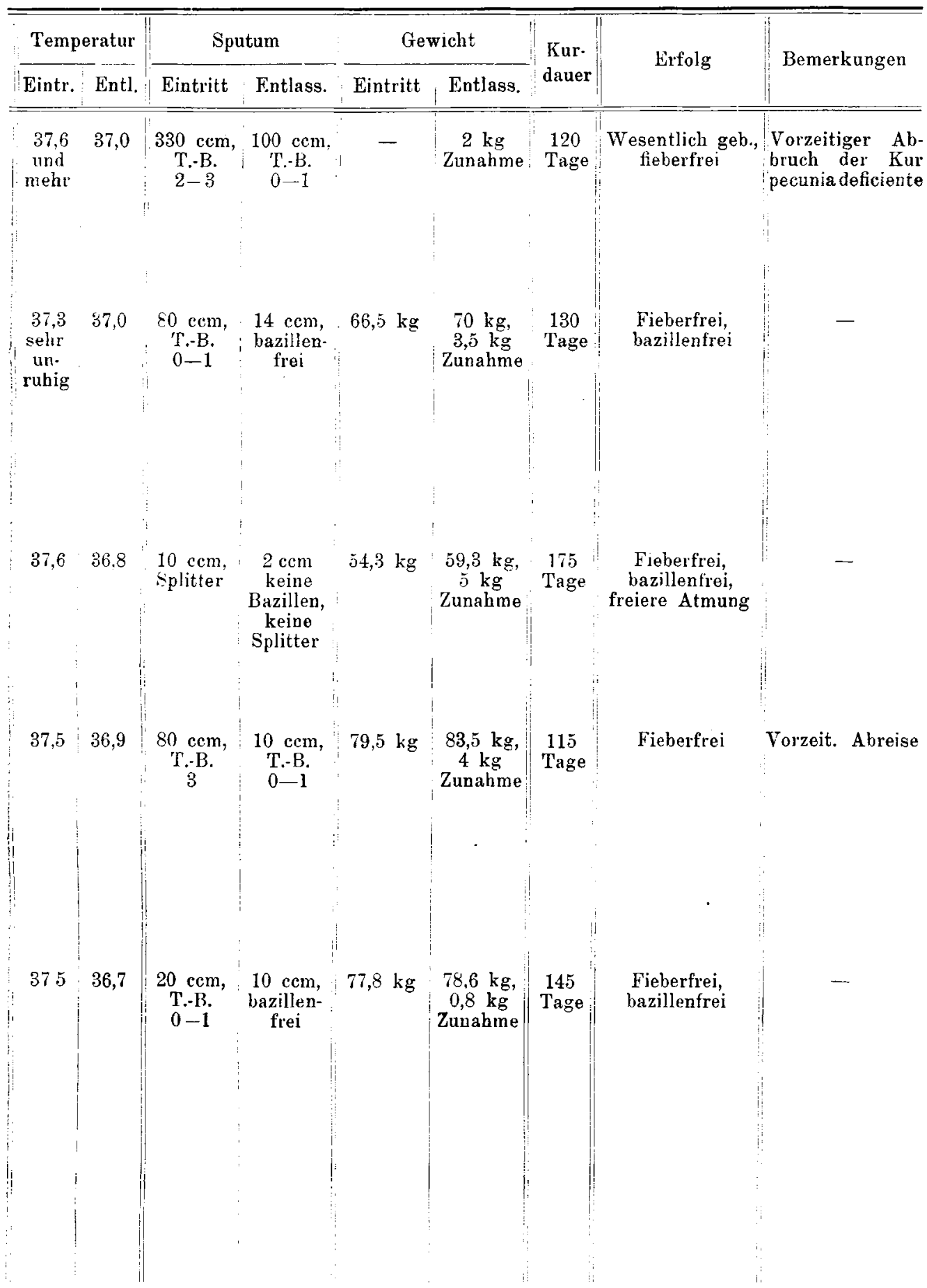




\begin{tabular}{|c|c|c|c|c|c|}
\hline \multirow{2}{*}{$\mathrm{Nr}$} & \multirow{2}{*}{$\begin{array}{l}\text { Name, } \\
\text { Alter, } \\
\text { Beruf }\end{array}$} & \multirow{2}{*}{ Anamnese } & \multirow{2}{*}{$\begin{array}{c}\text { Kom- } \\
\text { plikationen }\end{array}$} & \multicolumn{2}{|c|}{ Lungenbefund } \\
\hline & & & & Eintritt & Entlassung \\
\hline $108^{i}$ & $\begin{array}{l}\text { W., } \\
34 \text { J., } \\
\text { Offizier }\end{array}$ & $\begin{array}{l}\text { Beginn der Krankh. } \\
\text { vor } 21 / 2 \text { Jahren mit } \\
\text { Influenza, seitdem } \\
\text { Fieber u. Auswurf } \\
\text { mit T.B. zweimal } \\
\text { Kumyskur. Seit 9 } \\
\text { Mon.in Davos, 7Mon. } \\
\text { Tuberk.-Kur (Denys) } \\
\text { in einem Davoser } \\
\text { Sanatorium. Schlaf, } \\
\text { Appetit schlecht }\end{array}$ & Larynx & $\begin{array}{l}\text { L. vorn bis 3. Costa, } \\
\text { hinten bis MitteScap. } \\
\text { abnehm. Schallkürz. } \\
\text { L. Sp. scharf. Inspir., } \\
\text { Rhonchi angedeutet. } \\
\text { R. Schallkür. wie L. } \\
\text { In der Spitze hauch. } \\
\text { Exspir., Anblaseger. } \\
\text { nicht deutl. Rhonchi } \\
\text { feucht, mittelgrof, } \\
\text { vorn bis Basis, hinten } \\
\text { bis Angulus. Rausch. } \\
\text { überall, 3-5. Rippe } \\
\text { vorn Reiben }\end{array}$ & $\begin{array}{l}\text { L. Sp. hinten amphor. } \\
\text { reines Atmen. R. Sp. } \\
\text { ves.-bronch. Giemen. } \\
\text { M.-L. raubes Atmen }\end{array}$ \\
\hline 109 & $\begin{array}{l}\text { Sch., } \\
43 \text { J., } \\
\text { Musiker }\end{array}$ & $\begin{array}{l}\text { Krank seit } 1^{1} \text {. .ahr. } \\
\text { Letzten Winter in } \\
\text { Bett. Lungenspitzen- } \\
\text { katarrh. Appetit } \\
\text { mässig. Gewichts- } \\
\text { abnabme } 37 \text { Pfund. } \\
\text { Starke Atemnot }\end{array}$ & $\begin{array}{l}\vdots \\
\vdots \\
\vdots \\
\vdots\end{array}$ & $\begin{array}{l}\text { R. Lunge bis Basis } \\
\text { rel.Dämpf.O.-L.vorn } \\
\text { bronch.-amph.grosse } \\
\text { u. mittlere Rhonchi } \\
\text { halbkling. idem } \\
\text { Mittellappen. } \\
\text { L.-L. Crepitieren } \\
\text { hint.L. Sp kavernös, } \\
\text { klingende Rhonchi, } \\
\text { abwärts Krepitieren, } \\
\text { hinten idem } \\
\end{array}$ & $\begin{array}{l}\text { R. L. Dämpf. b. Basis, } \\
\text { nach unten abnebm. } \\
\text { O.-L. Tymp., amph. } \\
\text { Atmen, Exsp. hauch. } \\
\text { verläng., ke in e R h. } \\
\text { Bronchol. R vorn bis } \\
\text { zur } 3 . \text { hinten bis zur } \\
\text { 4. Rippe. L. Sp. Dmpf. } \\
\text { mit Tymp. bronch. } \\
\text { amph. Atmen m. verl. } \\
\text { hauch. Exspir. Bron- } \\
\text { choloquie vorn b. zur } \\
\text { 2. Rippe. Keine R h. } \\
\text { Über d. unt. Lungen- } \\
\text { teilen beiders. leises } \\
\text { Knatt. beim Inspir. }\end{array}$ \\
\hline 110 & $\begin{array}{c}\text { W., } \\
31 \dot{J}, \\
\text { Gutsbesitzer }\end{array}$ & $\begin{array}{l}\text { Beginn der Krankh. } \\
\text { vor } 6 \text { Jahren mit } \\
\text { Pleuritis dextra. Seit } \\
\text { 2 Jahren in Davos, } \\
\text { vor } 1 \text { Jahr Pneu- } \\
\text { monie, später Hä- } \\
\text { moptoe, danacb } 8 \mathrm{~kg} \\
\text { Abnahme. Schlaf } \\
\text { schlecht; lebhafte } \\
\text { Atembeschwerden }\end{array}$ & - & $\begin{array}{l}\text { R. Lunge schleppt, } \\
\text { steht beinahe stil]; } \\
\text { über der ganz. Lunge } \\
\text { starke Dämpf, von } \\
\text { Sp. bis Basis V, u. H. } \\
\text { feine u. mittl. knatt. } \\
\text { Rh., uber dem U.-L. } \\
\text { mehrals über d. O. L. } \\
\text { A tmung ves.-bronch. } \\
\text { L. intersc. Knattern, } \\
\text { O.-L. rauhes ves.- } \\
\text { bronch. Atmen }\end{array}$ & $\begin{array}{l}\text { R. Lunge atmet mit, } \\
\text { wenn auch weniger } \\
\text { als L. - Dämpf.über } \\
\text { der ganzen Lunge; } \\
\text { feine Rhonchi von } \\
\text { Spitze bis Basis. L. } \\
\text { O.-L. ves.-bronch. } \\
\text { Atmen }\end{array}$ \\
\hline 111 & $\begin{array}{l}\text { H., } \\
23 \text { J., } \\
\text { Errl. }\end{array}$ & $\begin{array}{l}\text { Krank seit } 3 \text { Jahren. } \\
\text { Beginn mit Erkalt. } \\
\text { Seit } 14 \text { Tagen hier in } \\
\text { Davos. Liegekur }\end{array}$ & 一 & $\begin{array}{l}\text { L.O.-L.tymp. Dämpf. } \\
\text { Grosseu.mittl.kling. } \\
\text { u. halbkling. Rhonchi } \\
\text { vorn u. hinten. U.-L. } \\
\text { vereinz. Krepitieren. } \\
\text { R. Sp. ves.-bronch. } \\
\text { Atmen }\end{array}$ & $\begin{array}{c}\text { Über dem L. O.-I. } \\
\text { noch trock. Rhonchi } \\
\text { ohne Klang; sonst } \\
\text { frei }\end{array}$ \\
\hline
\end{tabular}




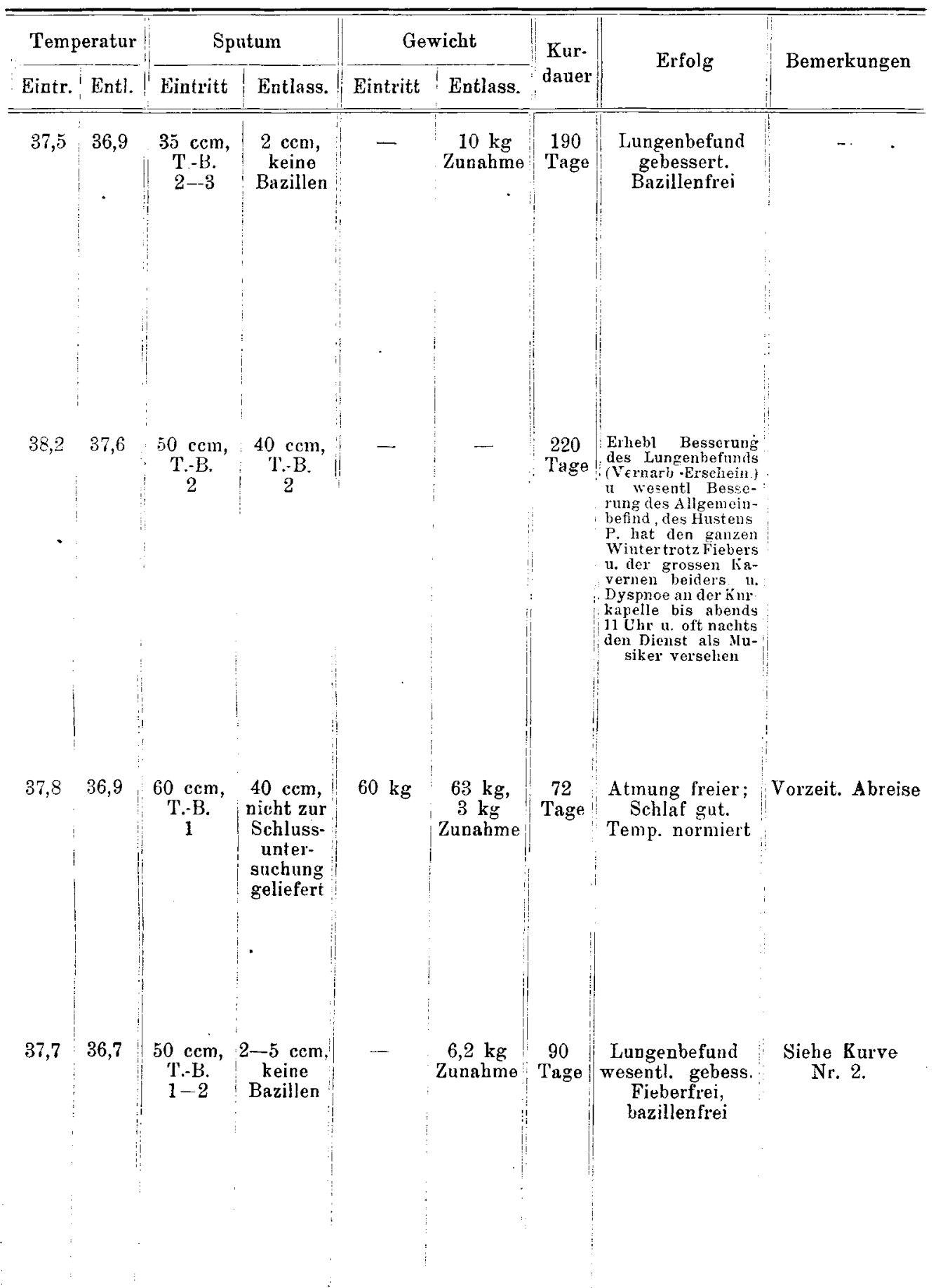




\begin{tabular}{|c|c|c|c|c|c|}
\hline \multirow{2}{*}{$\mathrm{Nr}$. } & \multirow{2}{*}{$\begin{array}{l}\text { Name, } \\
\text { Alter, } \\
\text { Beruf }\end{array}$} & \multirow{2}{*}{ Anamnese } & \multirow{2}{*}{$\begin{array}{c}\text { Kom- } \\
\text { plikationen }\end{array}$} & \multicolumn{2}{|c|}{ Lungenbefund } \\
\hline & & & & Eintritt & Entlassung \\
\hline 112 & $\begin{array}{c}\text { St., } \\
29 \text { J., } \\
\text { Hotel- } \\
\text { hausknecht }\end{array}$ & $\begin{array}{c}\text { Krank seit } 2 \text { Jahren. } \\
\text { Beginn mitSchweiss, } \\
\text { Frost und Lungen } \\
\text { katarrh. In letzter } \\
\text { Zeit } 4 \text { Pfd. Gewichts- } \\
\text { abnahme. }\end{array}$ & - & $\begin{array}{l}\text { Über beiden Spitzen } \\
\text { rel. Dämpf., rechts } \\
\text { über dem ganz. O-L. } \\
\text { L. Sp. leises ves. } \\
\text { Bronchialatmen und } \\
\text { trock. Rhonchi. R. } \\
\text { O.-L schleppt, über } \\
\text { der Spitze trockene } \\
\text { Rhonchi }\end{array}$ & $\begin{array}{l}\text { Keine Rhonchi mehr, } \\
\text { über der R. Sp. nur } \\
\text { noch etwas Knacken }\end{array}$ \\
\hline 113 & $\begin{array}{l}\text { T., } \\
32 \text { J., } \\
\text { Student }\end{array}$ & $\begin{array}{l}\text { Mutter an T.-B. }{ }^{\dagger} \\
\text { Vor } 8 \text { Jahr. Pleuritis, } \\
\text { seit } 5 \text { Jahren lungen- } \\
\text { krank, in letzt. Jahr. } \\
20 \mathrm{~kg} \text { Gerichtsabn. } \\
\text { Hautige Blutungen. } \\
\text { Fieber. } 2 \text { Jahre Auf- } \\
\text { enthalt in einem Da- } \\
\text { voser Sanatorium. } \\
2 \text { kg Gewichtszu- } \\
\text { nahme. Lungenbef. } \\
\text { verschlechteit. Seit } \\
2 \text { Jahren beiser }\end{array}$ & $\begin{array}{l}\text { Nierentuberkul. } \\
\text { Arteriosklerose. } \\
\text { Im Urin Bazillen } \\
\text { nachgewiesen. } \\
\text { Eiweis } 5 \% \text { \% }\end{array}$ & $\begin{array}{l}\text { L. O.-L. tymp. Dmpf. } \\
\text { Br. amph. Atmen mit } \\
\text { klingend. Rhonchi, } \\
\text { Kavernoloquie, An- } \\
\text { blasegeräusch, U. L. } \\
\text { krepitieren hint. an } \\
\text { der Basis an um- } \\
\text { schrieb. Stelle kling. } \\
\text { Rhonchi u. Kavernol. } \\
\text { R. O.-L. ves.-bronch. } \\
\text { rauh, U.-L. rauhes } \\
\text { Atmen, Rauschen }\end{array}$ & $\begin{array}{l}\text { Relat. Dämpf. über } \\
\text { beiden Spitzen. L.im } \\
\text { Bereich des O.-L. mit } \\
\text { Spur Tympanie. L } \\
\text { O.-L.metall. Knaren } \\
\text { u. etwas Giemen. U.- } \\
\text { L. vorne Atmungs- } \\
\text { geräusch durch kre. } \\
\text { pitierende Rhonchi } \\
\text { verdeckt, ebens.hint. } \\
\text { R. O.-L. ves..bronch. } \\
\text { Atmen, keine Rh. }\end{array}$ \\
\hline 114 & $\begin{array}{l}\text { M., } \\
26 \mathrm{~J}_{.,} \\
\text {Privatier }\end{array}$ & $\begin{array}{l}\text { Seit 2 Mon. krank, } \\
\text { Beginn mitInfluenza, } \\
\text { Pleuritis sin.exsud., } \\
\text { Pneumonie. Husten, } \\
\text { Auswurf, T.-B. - } \\
\text { Nachtschweisse, die } \\
\text { in letzter Zeit sehr } \\
\text { stark sind. Zu Hause } \\
\text { I.K.-Injektionen, wo- } \\
\text { nach Verminderung } \\
\text { des Fiebers }\end{array}$ & $\begin{array}{l}\text { Starke } \\
\text { Anämie }\end{array}$ & $\begin{array}{l}\text { L. Lunge schleppt : Ex- } \\
\text { sudat vorne bis 4. Rippe, } \\
\text { hint. bis Mitte Scapulae. } \\
\text { o.-L. rel. Dämnf., nach } \\
\text { unten hin absolut. O,-L. } \\
\text { scharf ves. Atmen, U.-L. } \\
\text { fastod.ganz aufgehoben. } \\
\text { R. O.-L. rel, Dämpf. mit } \\
\text { Tympanie, bronch -amph. } \\
\text { Atm, Kavernoloquie in } \\
\text { ganzen O.-L, klingende, } \\
\text { aber spärliche Rhonchi. } \\
\text { U.-L.: Giemen u Pfeifen, } \\
\text { ves.-bronch. dtmen } \\
\end{array}$ & $\begin{array}{l}\text { L. Lunge abgeschw. } \\
\text { Atmen. - Exsudat } \\
\text { resorbiert. R. Spitze } \\
\text { Retraktion, bronch.- } \\
\text { amph. Atmung, zäh } \\
\text { klingendes Knarren }\end{array}$ \\
\hline 115 & $\begin{array}{l}\text { P.. } \\
\begin{array}{l}35 \\
\text { Frau }\end{array}\end{array}$ & $\begin{array}{l}\text { Krank geit } 3 \text { Jahren. } \\
\text { Wiederholte spezif. } \\
\text { Behandlung ohne } \\
\text { wesentlichen Erfolg. } \\
\text { Darmstörungen }\end{array}$ & $\begin{array}{c}\text { Darmtuber- } \\
\text { kulose (Stuhl } \\
\text { T.-B. }=+ \text { +) }\end{array}$ & $\begin{array}{l}\text { Über beiden O.-L. rel. } \\
\text { Dämpf., ves.-bronch. } \\
\text { Atmen, knatternde } \\
\text { Rhonchi beiderseits, } \\
\text { auch über den U.-L. }\end{array}$ & $\begin{array}{l}\text { Klopfschall aufge- } \\
\text { hellt, Atmung ves. } \\
\text { bronch. Nirgends } \\
\text { mehr Rh on chi,in } \\
\text { den Spitzen spärlicb. } \\
\text { leises Knistern (Er- } \\
\text { offnungsknistern) }\end{array}$ \\
\hline 116 & $\begin{array}{c}\text { K." } \\
27 ., \\
\text { Kaufmann }\end{array}$ & $\begin{array}{l}\text { Seit } 2 \text { Jahren krank. } \\
\text { Kur in Görbersdorf, } \\
\text { gebeilt entl., nach } \\
\text { einig. Monaten Blu- } \\
\text { tung. In Davos } \\
\text { seit } 2 \text { Monaten; } \\
\text { viel Liegekur. Hust. } \\
\text { Schwäche, Apetit- } \\
\text { mangel. }\end{array}$ & Otitis media & $\begin{array}{l}\text { Relative Dämpfung } \\
\text { beid. Spitz.; rauhes } \\
\text { ves.bronch. Atmen } \\
\text { hinten beiders., ein- } \\
\text { zelne Rhonchi } \\
\text { L. Unterlappen rauh, } \\
\text { sakkad. ves.-bronch. } \\
\text { Atmen, Mittellappen } \\
\text { scharf ves.-broncho- } \\
\text { bronchal. }\end{array}$ & $\begin{array}{l}\text { Keine Rhonchi, über } \\
\text { den Spitz. Narben- } \\
\text { atmen }\end{array}$ \\
\hline
\end{tabular}




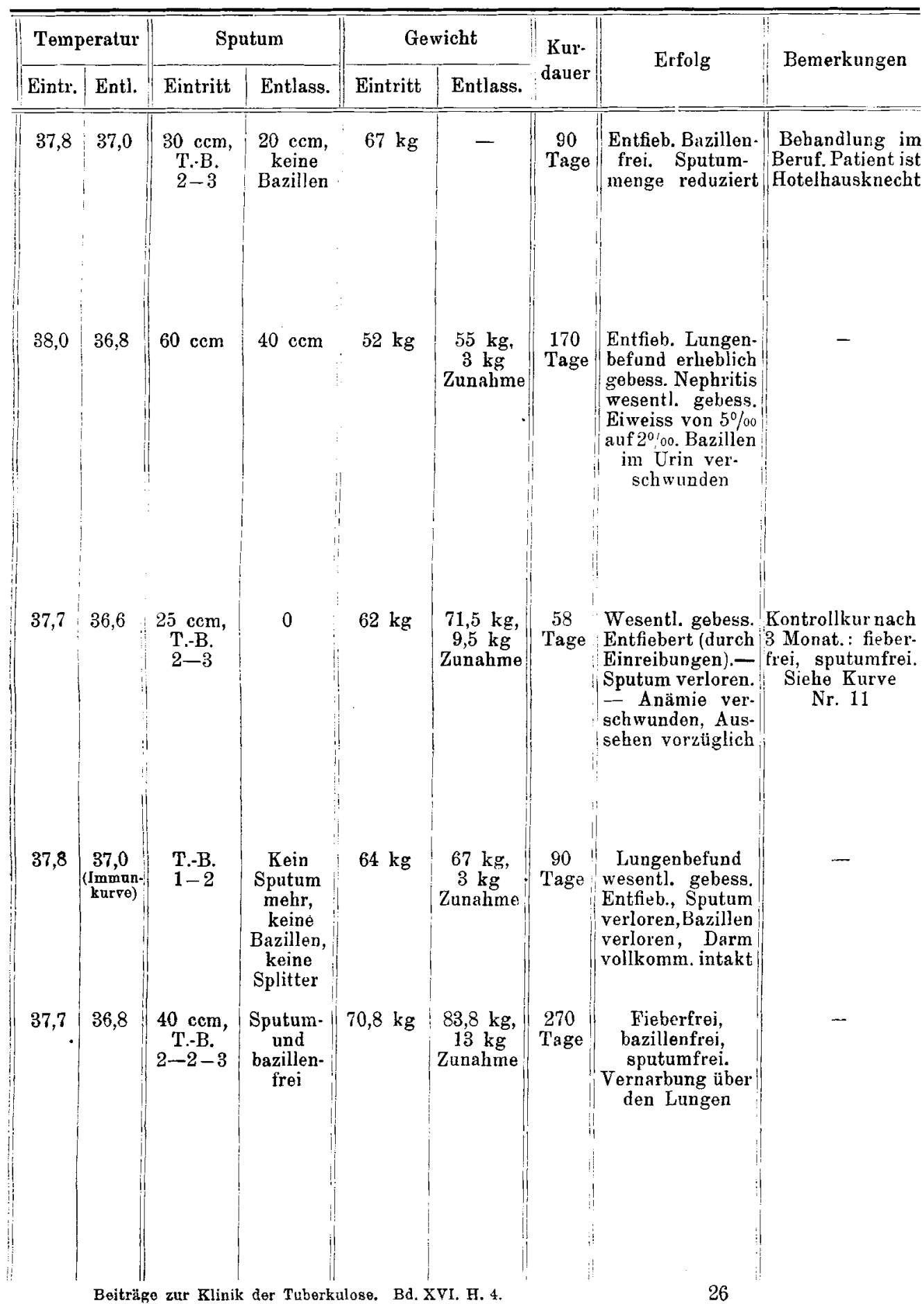

Beiträge zur Klinik der Tuberkulose. Bd. XVI. ‥ 4. 


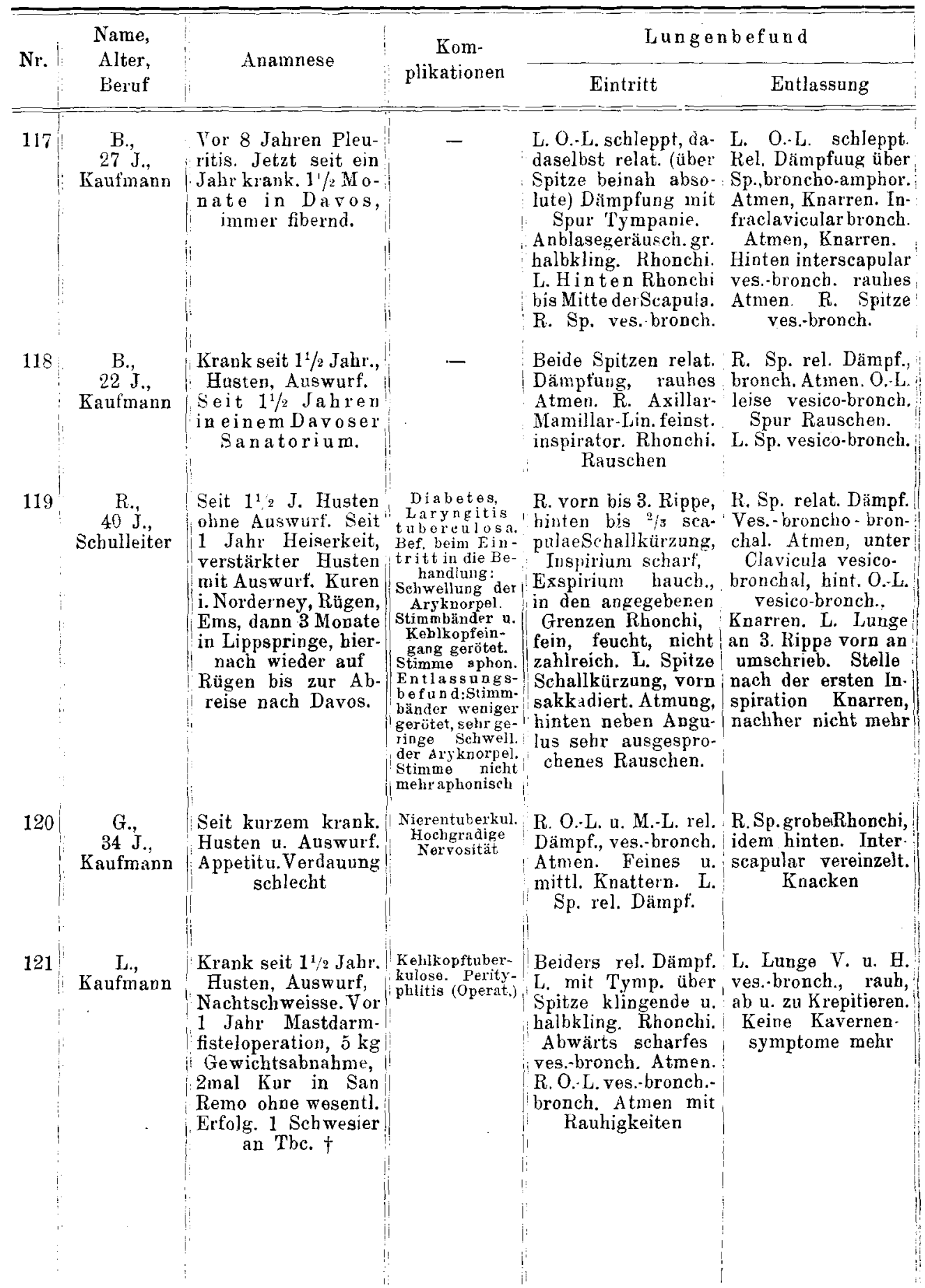




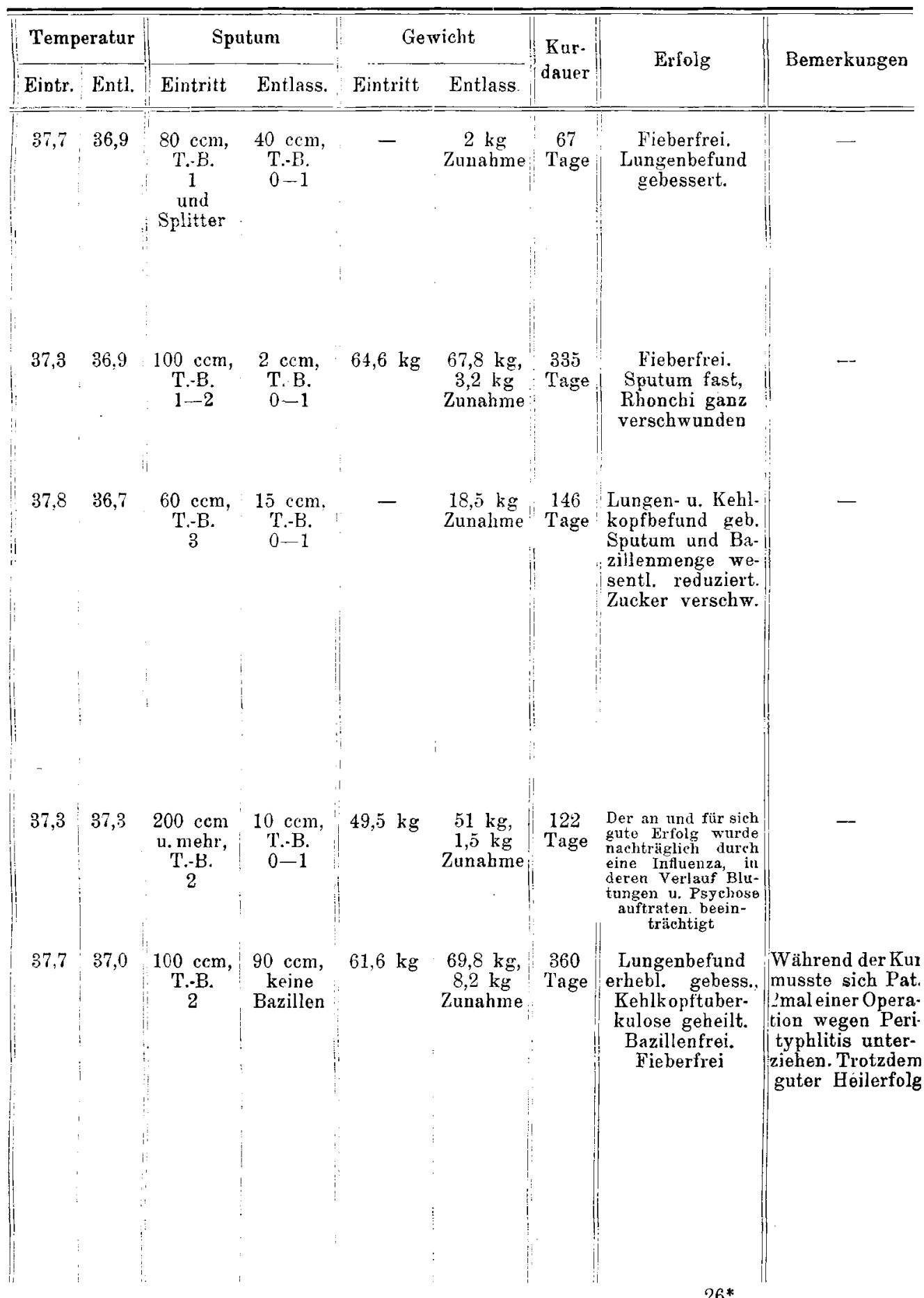




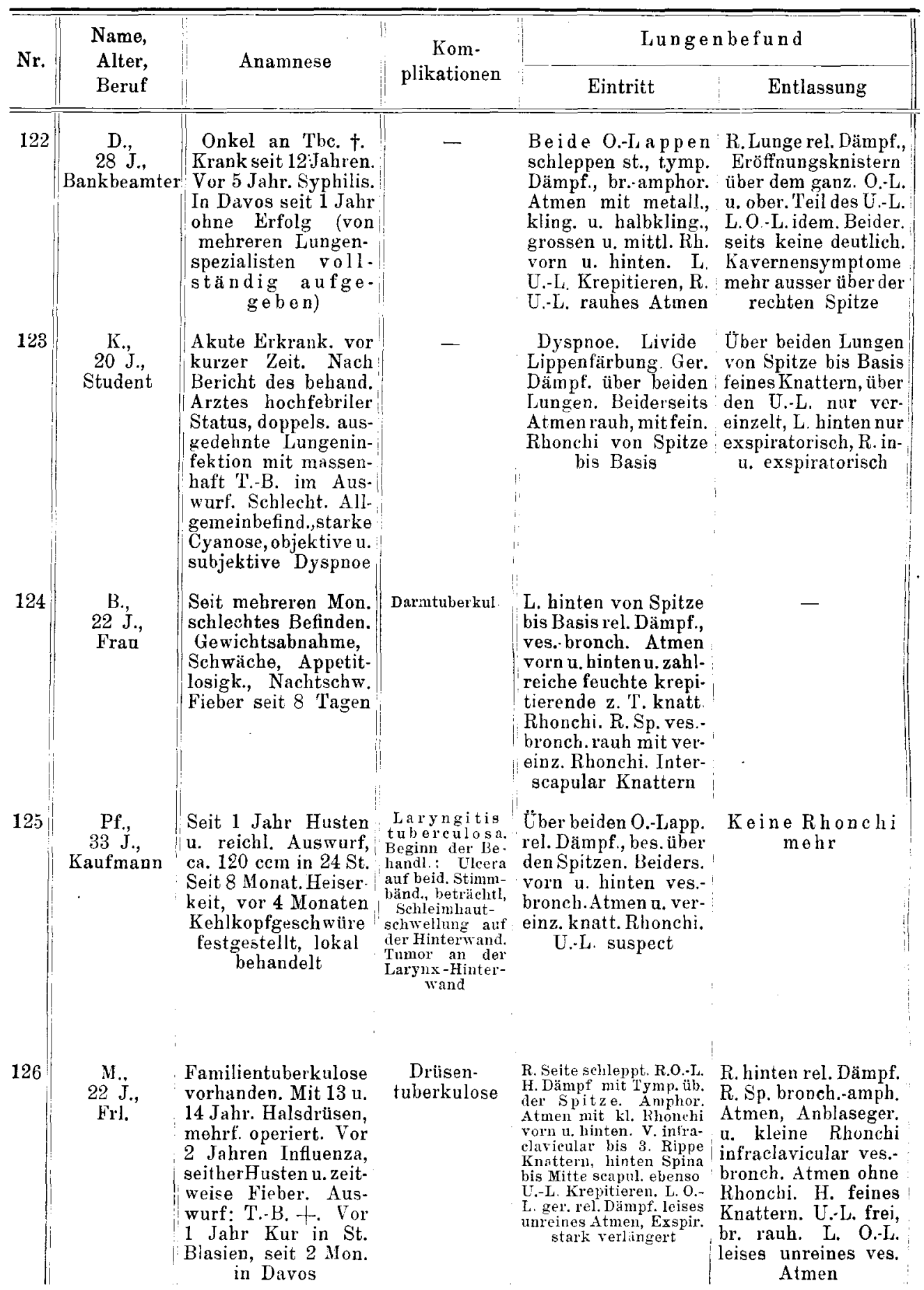




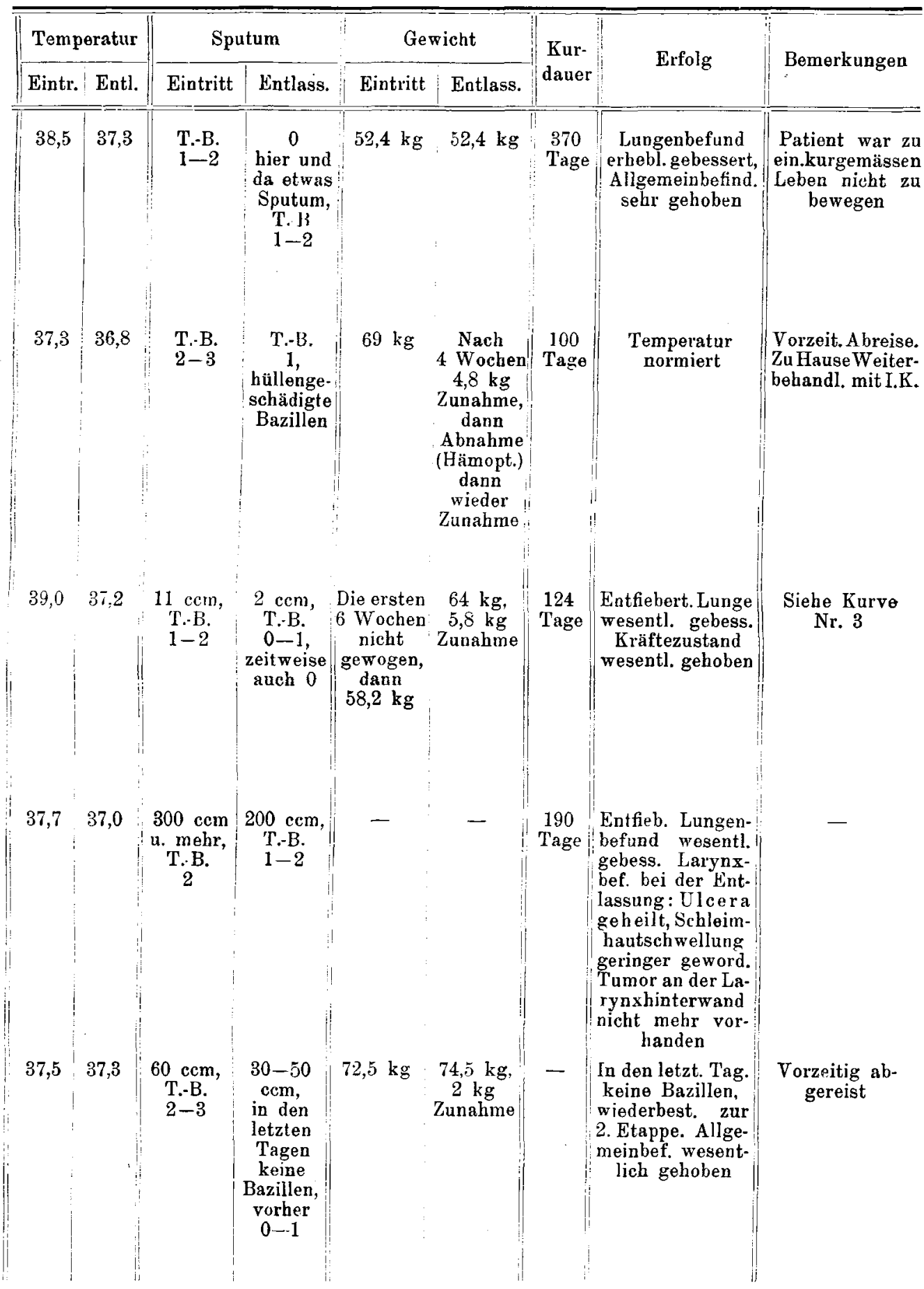




\begin{tabular}{|c|c|c|c|c|c|}
\hline \multirow{2}{*}{ Nr. } & \multirow{2}{*}{$\begin{array}{l}\text { Name, } \\
\text { Alter, } \\
\text { Beruf }\end{array}$} & \multirow{2}{*}{ Anamnese } & \multirow{2}{*}{$\begin{array}{c}\text { Kom- } \\
\text { plikationen }\end{array}$} & \multicolumn{2}{|c|}{ Lungenbefund } \\
\hline & & & & Eintritt & Entlassung \\
\hline 127 & $\begin{array}{c}\text { K., } \\
26 \text { J., } \\
\text { Pfarrer }\end{array}$ & $\begin{array}{l}\text { Krank seit } 1^{1 / 2} \text { Jahr. } \\
\text { Lungenspitzen- } \\
\text { katarrh festgestellt. } \\
1 \text { Jahr vorher Peri } \\
\text { proktitis, Operation, } \\
\text { seither } 3 \text { eiternde } \\
\text { Fisteln }\end{array}$ & $\begin{array}{l}\text { Anal-u. Peri- } \\
\text { neal-Fisteln } \\
\text { mit reichlich. } \\
\text { Sekretion, im } \\
\text { Sekret zahl- } \\
\text { reicheSplitter }\end{array}$ & $\begin{array}{l}\text { Über beiden Sp. rel. } \\
\text { Dämpf. ves.-bronch. } \\
\text { Atmen, Knattern, R. } \\
\text { stärker als L. U..L. } \\
\text { rauh. Atmen, bes. R. }\end{array}$ & $\begin{array}{l}\text { Über beiden Sp. rel. } \\
\text { Dämpf. R. stärker } \\
\text { als L. Ves.bronch. } \\
\text { Atm. ohne Rhonchi. } \\
\text { Spur Rauhigkeiten } \\
\text { vorn. R. Sp. hinten } \\
\text { Kavernoloquie, br.- } \\
\text { amph. Atmen, ver- } \\
\text { einz. Knattern, sonst } \\
\text { nirgends Rhonchi }\end{array}$ \\
\hline 128 & $\begin{array}{c}\text { G., } \\
36 \text { J., } \\
\text { Pharmazeut }\end{array}$ & $\begin{array}{l}\text { Vater u. Mutter an } \\
\text { Tbc. †. Krank seit } \\
1 / 2 \text { Jahr. Beginn mit } \\
\text { Influenza, vom Haus- } \\
\text { arzt Spitzenkatarrh } \\
\text { festgestellt }\end{array}$ & $\begin{array}{c}\text { Darm. } \\
\text { tuberkulose. } \\
\text { Im Stuhl T.- } \\
\text { B. }=+. \\
\text { Diarrhöen }\end{array}$ & $\begin{array}{l}\text { R. Spitze rel. Dämpf. } \\
\text { les.-bronch. A tmen } \\
\text { mit feinsten insp. Rh. } \\
\text { L. O.-L. rel. Dämpf. } \\
\text { ves.-bronch. Atmen, } \\
\text { lautes Knistern mit } \\
\text { Rauschen, V. mit } \\
\text { halbkling. Knacken. } \\
\text { U.-L. ves.-bronch. u. } \\
\text { Rauschen }\end{array}$ & $\begin{array}{l}\text { Rel. Dämpf. üb. beid. } \\
\text { Spitzen. R. Sp. ves.- } \\
\text { bronch. Atmen, hint. } \\
\text { feines Knistern, An- } \\
\text { blasegeräusch. L. Sp. } \\
\text { ves.bronch.Rausch., } \\
\text { hinten Broncholoqu. } \\
\text { vorn vereinz. Rh. }\end{array}$ \\
\hline
\end{tabular}

\section{Etappen-} K, Frl.
I. Etappe: Krank seit $4^{\mathrm{i}} \mathrm{q} \mathbf{\mathrm { J }} \mathrm{ahr}$. Vor 2 Jahr. in Davos mit PTO. behandelt mit gutem Erfolg. ZuHause vor $1 / 2$ Jahr Influenza, seither Fieber bis 38,5 . Sputum $175 \mathrm{ccm}$, Schlaf, A ppetit schlecht.

Schmerzen im Hals. Dyspnoe. Nacht. scliweisse. Cyanose.

\section{Etappe}

(10 Monate später): Befind. zu Hause gut, Schlaf, Appetit gut. Temperatur hat sich normiert
Laryngitis tuberculosa. Ulcerationen an beiden Stimmbänd.
L.O.L.tymp.Dämpf. amph. Atmen, kling. Tropfengeräusche vorn u. hinten. $R$. Sp. hinten bronch.amph. rauh. Atmen, interscap. Knistern. Vorn Atmung ves. bronch., im 2. Interkostalraum Knistern

L. Lunge rel. Dämpf. L. Lunge st. geschr. unbestimmtes, abge- Rhonchi selten. R. schw. rauh. Atmen, Spitze geschrumpft. hinten interscapular Kavernol. Rauhig. einige Rhonchi. R. keiten, keine Rh. Spitze ves.-bronch. $b$. bronch., ohne Rh. 


\begin{tabular}{|c|c|c|c|c|c|c|c|c|}
\hline \multicolumn{2}{|c|}{ Temperatur } & \multicolumn{2}{|c|}{ Sputum } & \multicolumn{2}{|c|}{ Gewicht } & \multirow{2}{*}{$\begin{array}{c}\text { Kur- } \\
\text { dauer }\end{array}$} & \multirow{2}{*}{ Erfolg } & \multirow{2}{*}{ Bemerkungen } \\
\hline Eintr. & Fintl. & Eintritt & Entlass. & Eintritt & Entlass. & & & \\
\hline 37,8 & 37,1 & $\begin{array}{c}10-20 \\
\text { ccm, } \\
\text { T. }-B \\
2-3\end{array}$ & $\begin{array}{c}0-2 \mathrm{ccm} \\
\mathrm{T} \cdot \mathrm{B} \\
0-1\end{array}$ & $62,4 \mathrm{~kg}$ & $\begin{array}{c}64,4 \mathrm{~kg} \\
2 \mathrm{~kg} \\
\text { Zunahme }\end{array}$ & - & $\begin{array}{c}\text { Entfiebert. } \\
\text { Sput.-Abnahme. } \\
\text { Bazill.-Abuahme. } \\
\text { Fisteln fast } \\
\text { geheilt. Keine } \\
\text { Beschwerden }\end{array}$ & $\begin{array}{l}\text { Zur 2. Etappe } \\
\text { wiederbestellt }\end{array}$ \\
\hline 37,5 & 37,0 & $\begin{array}{c}30 \text { ccm, } \\
\text { T.-B. } \\
0-1 \\
\text { Splitter }\end{array}$ & $\begin{array}{c}15 \text { ccm, } \\
\text { keine } \\
\text { Bazillen, } \\
\text { keine } \\
\text { Splitter }\end{array}$ & $57 \mathrm{~kg}$ & $\begin{array}{c}61 \mathrm{~kg} \\
4 \mathrm{~kg} \\
\text { Zunatime }\end{array}$ & 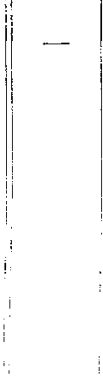 & $\begin{array}{l}\text { Entfieb. Bazillen } \\
\text { in Sputum und } \\
\text { Stuhl verschw. } \\
\text { Keine Durchfälle } \\
\text { mehr }\end{array}$ & - \\
\hline
\end{tabular}

fall.

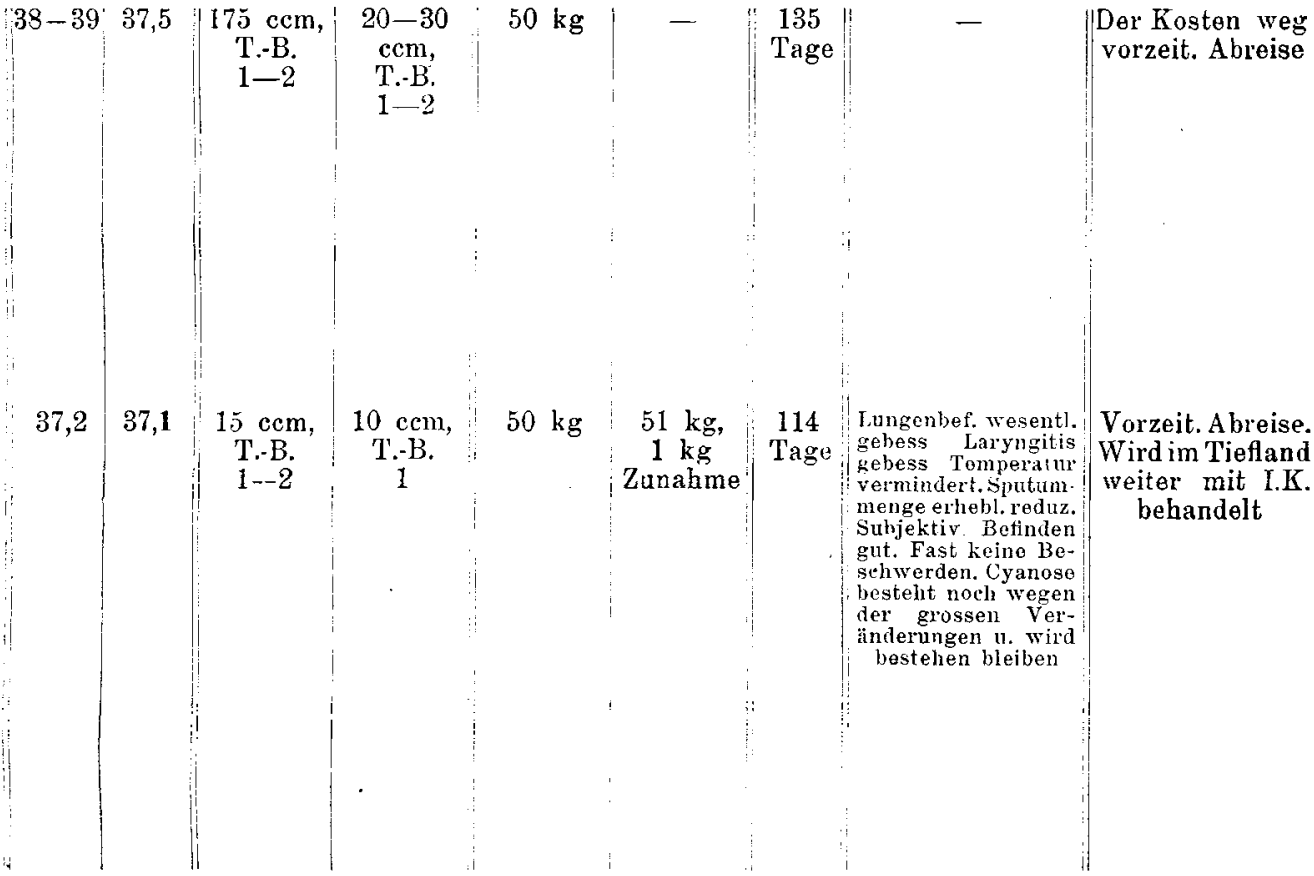




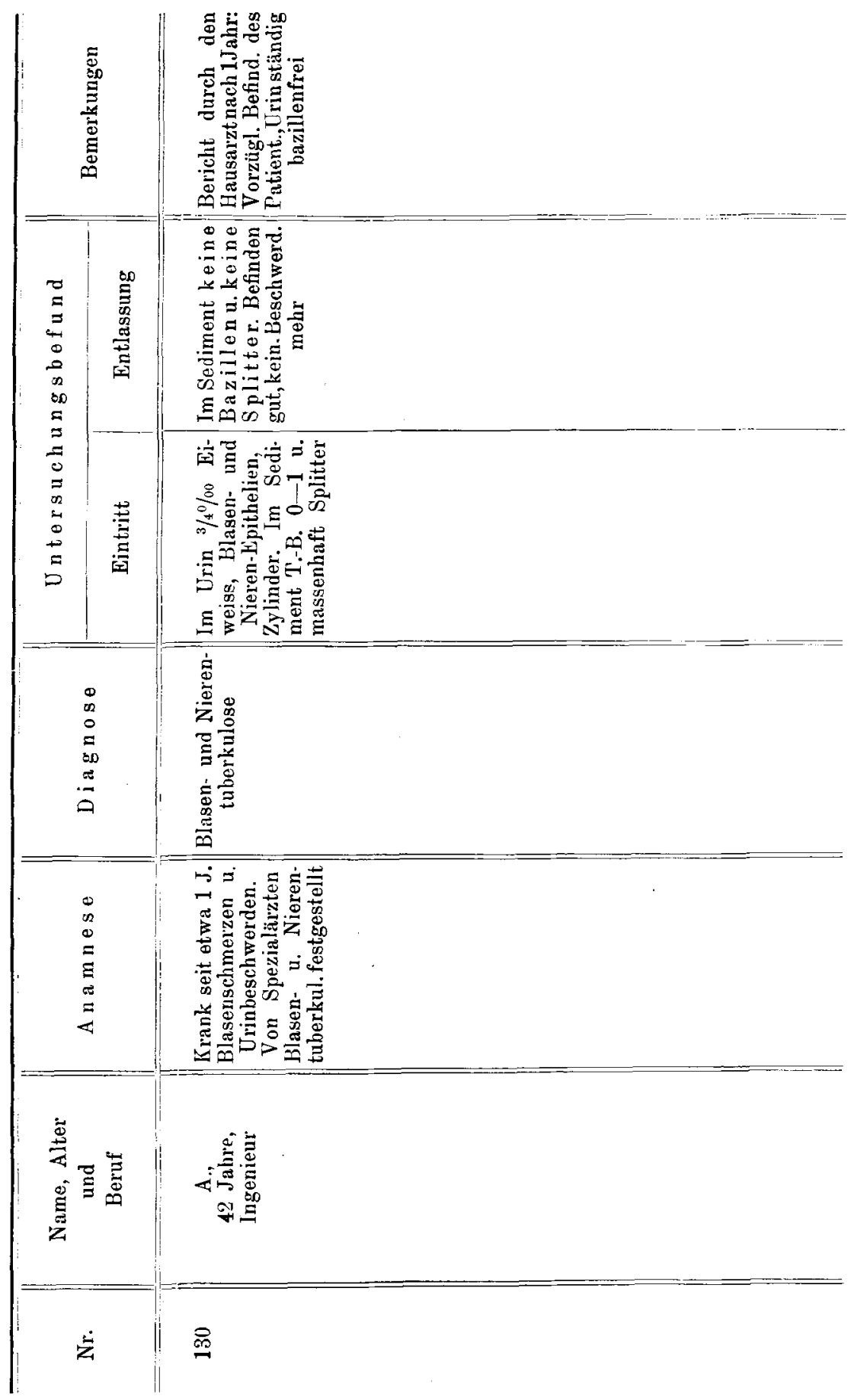


Als weiteren Beweis, dass I.K. nicht nur im Hochgebirge, sondern auch im Tiefland seine volle Wirkung entfaltet, fügen wir unserer Veröffentlichung vergleichsweise folgende 46 uns freundlichst überlassenen Fälle von Dr. Pumr, Bahnarzt in Böhmen, bei.

Die Wirkung des IK. bei chirurgischen Tuberkulosefällen zeigt die nachstehende Arbeit von Dr. Westphal (Hannover), die er liebenswürdigerweise Dr. C. Spengler zur Verfügung gestellt hat. Die hier angeführten Fälle sind insofern interessant und für die spezifische antituberkulöse Wirkung des I.K. beweisend, als in dem Gonitisfall ein chirurgischer Eingriff, der als notwendig erklärt worden war, infolge der Wirksamkeit der I.K.-Behandlung unnötig und dem Patienten die Beweglichkeit des, Kniegelenks erhalten wurde, und bei der Bauchfelltuberkulose der I.K.-Behandlung eine Laparotomie voranging, die über die Schwere und operative Unheilbarkeit des Leidens kaum einen Zweifel liess.

Die Vorschläge Westpha]s, die Pausen zwischen den einzelnen um Teilstriche gesteigerten I.K.-Dosen auf Wochen zu bemessen, entsprechen der Geptlogenheit Dr. C. Spenglers im Beginn der Behandlung, wo bis acht- und vierzehntägige Pausen oft nützlich sind. Dagegen steigert Dr. Spengler im weiteren Verlauf die Dosen schneller und begnügt sich auch oft mit mehrtägigen, allerdings individuell abgestuften Pausen. 

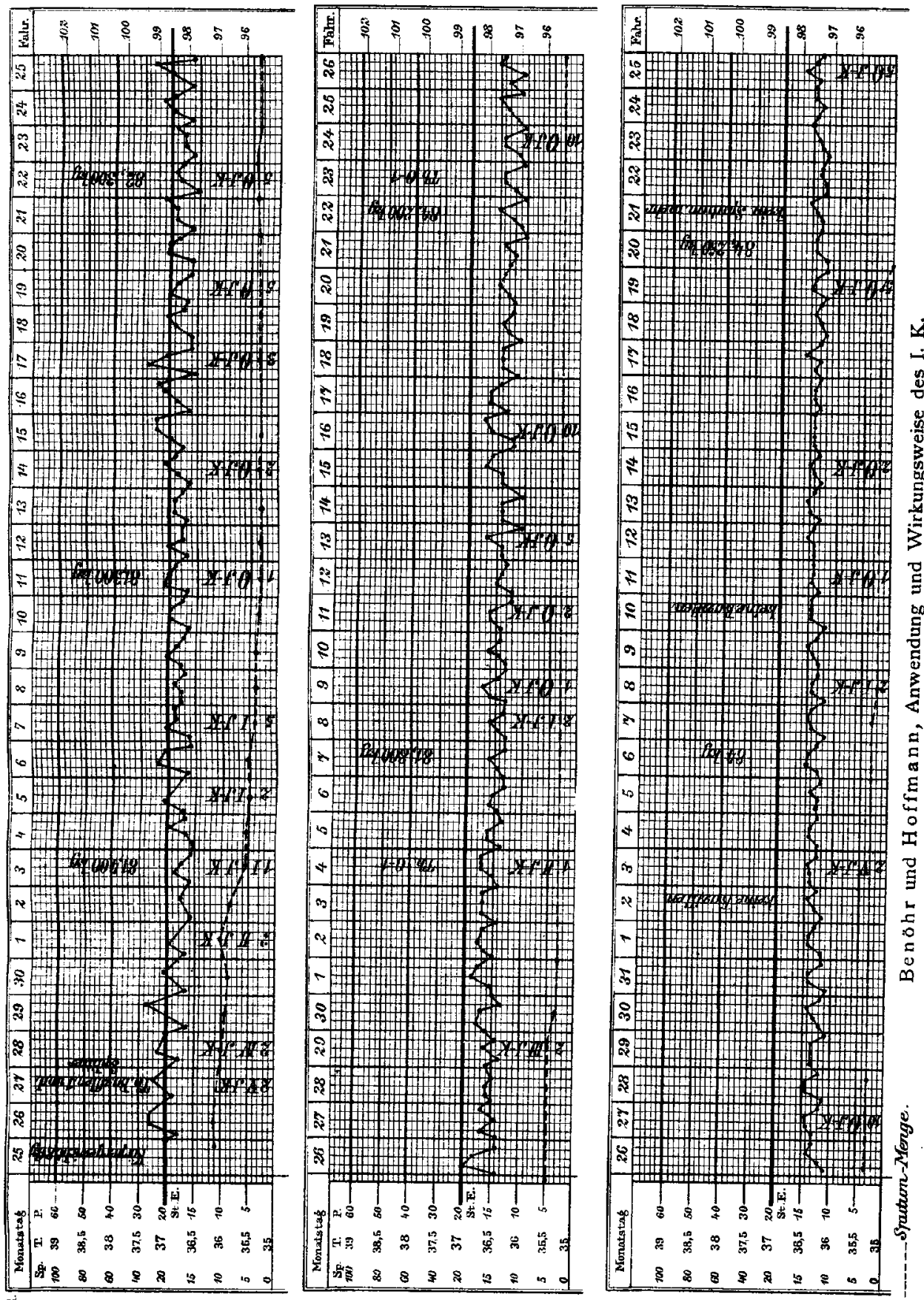

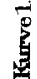




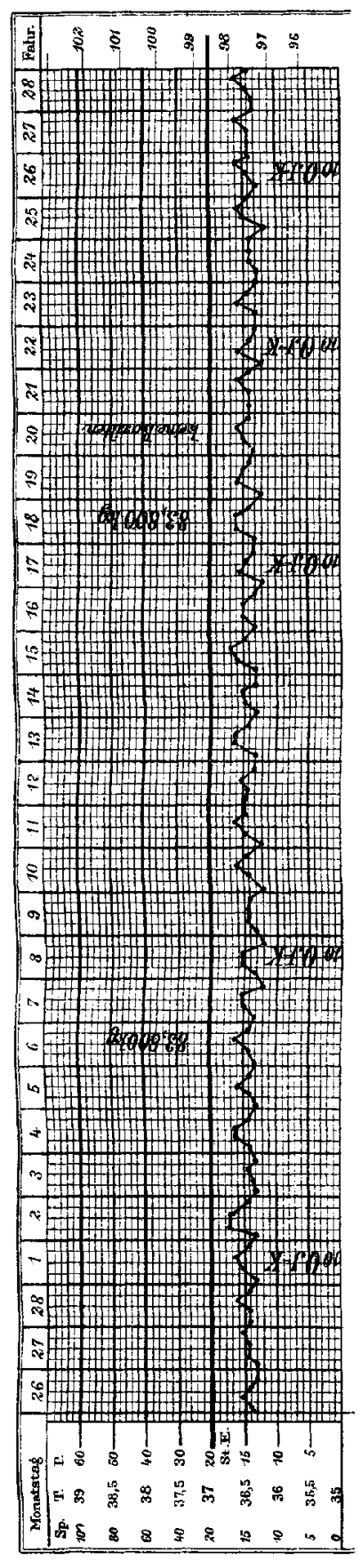

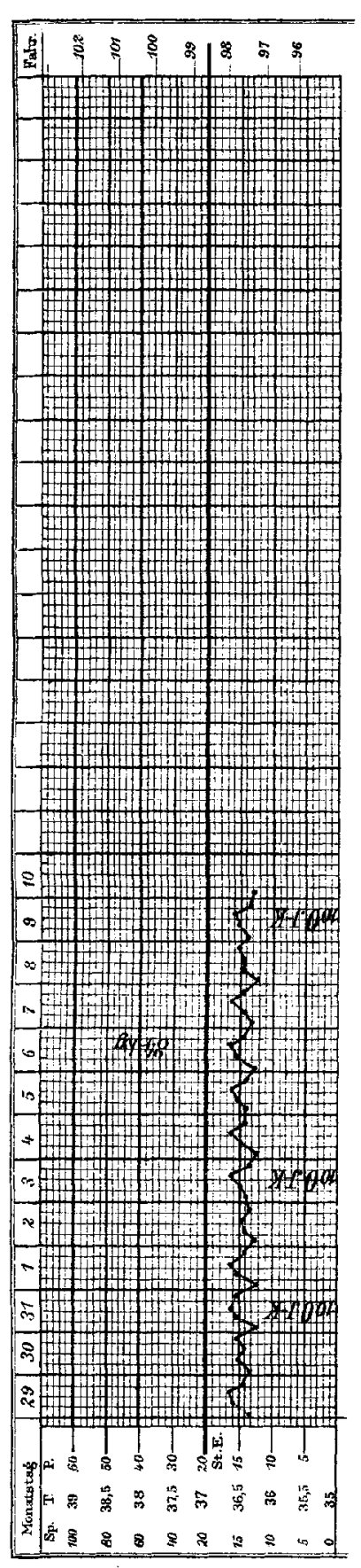

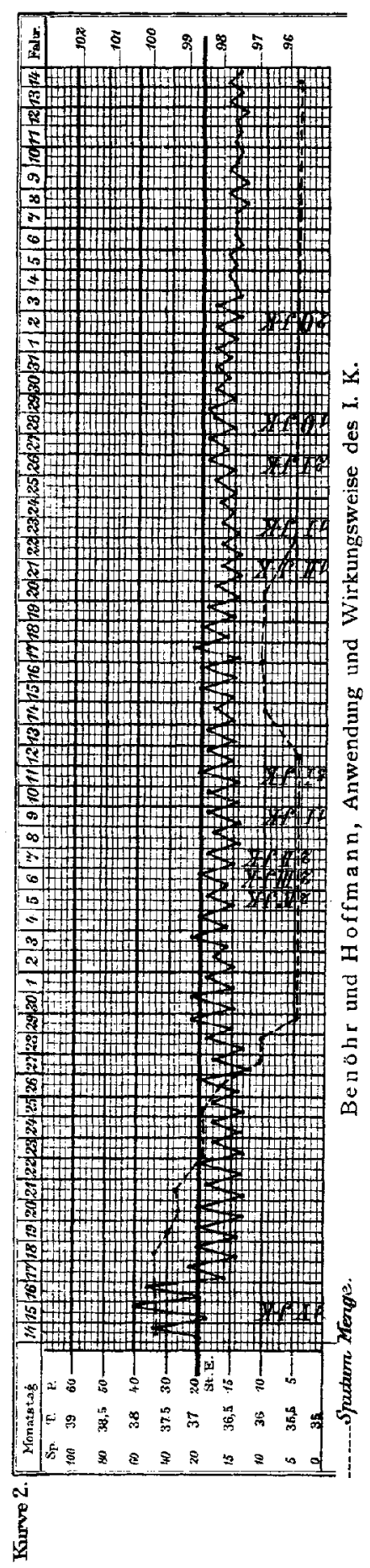




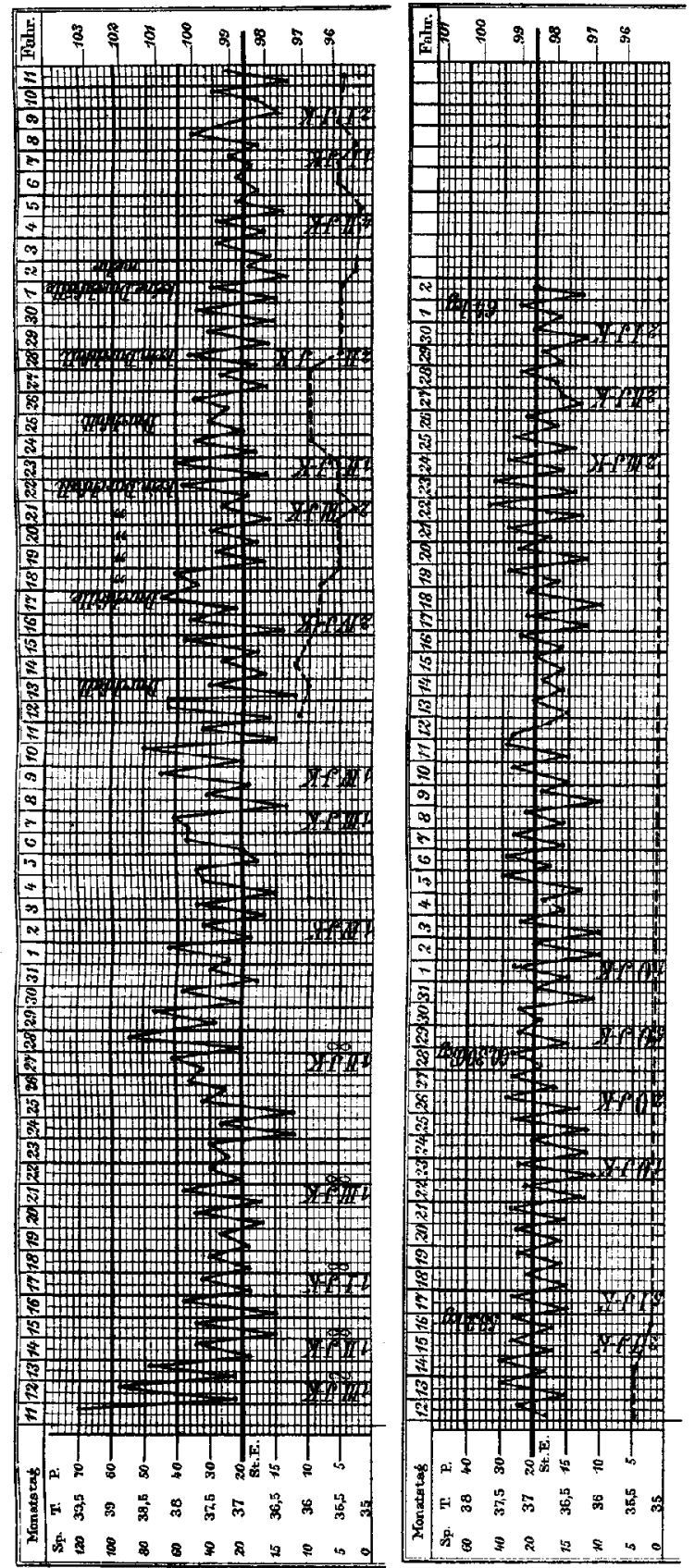

है

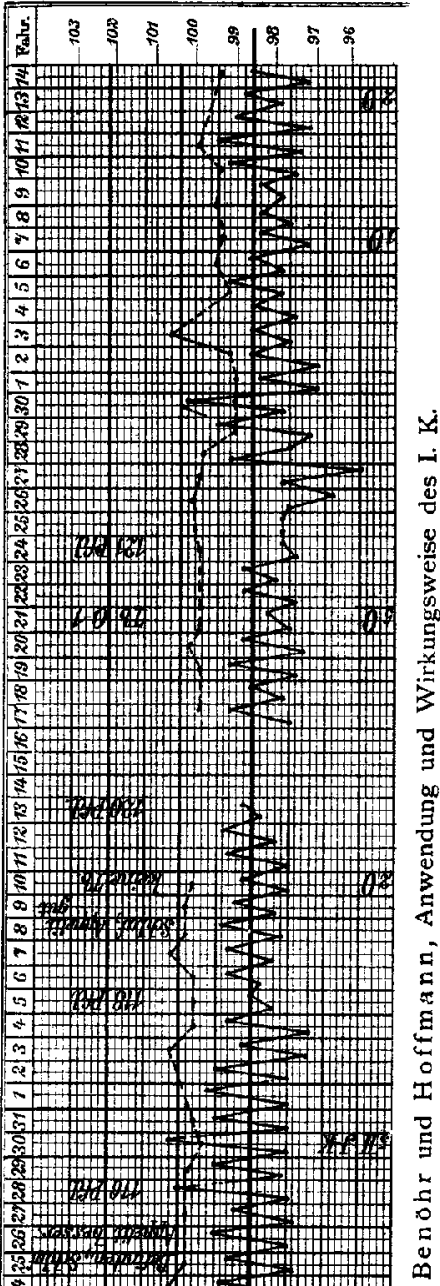

計 亭

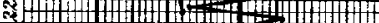
ז̀ $\mathrm{m}$ $\grave{s} \mathrm{~m}+\mathrm{m}+$ 决 尺 地

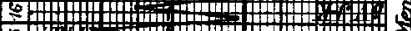

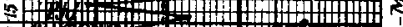
5 \&i:

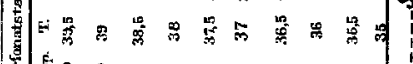

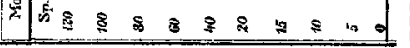
i 要

Kgl. Univ.Druckerei H. Startz A. G., Worzburg. 


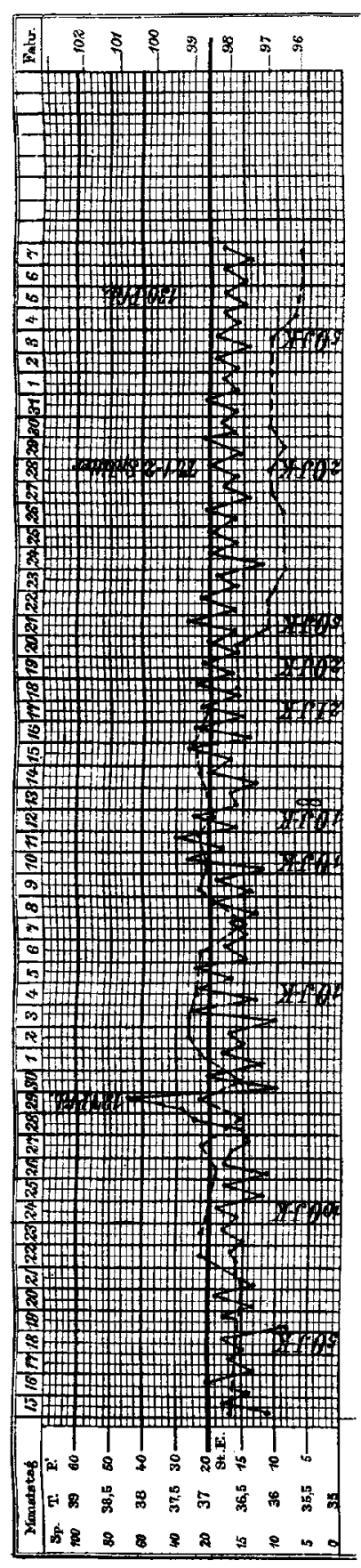

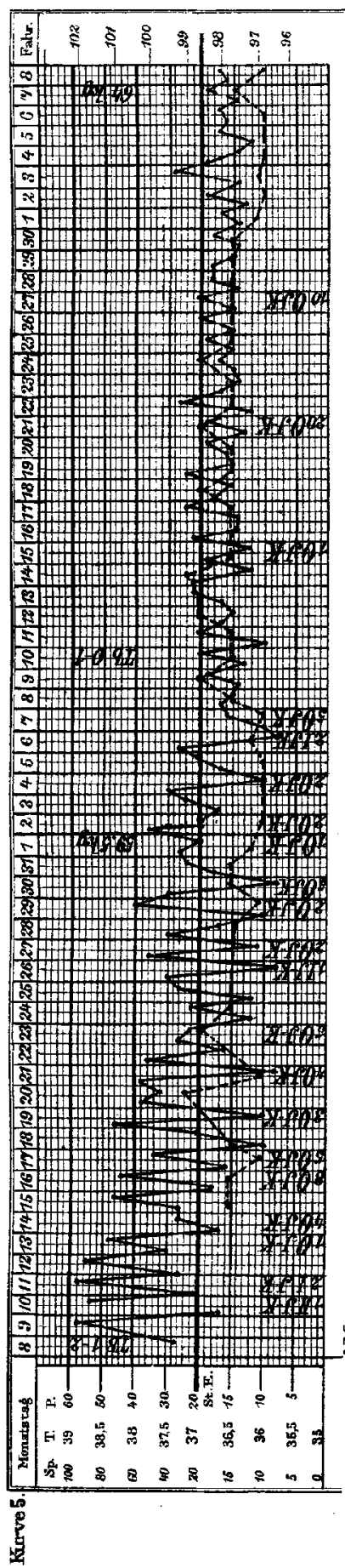

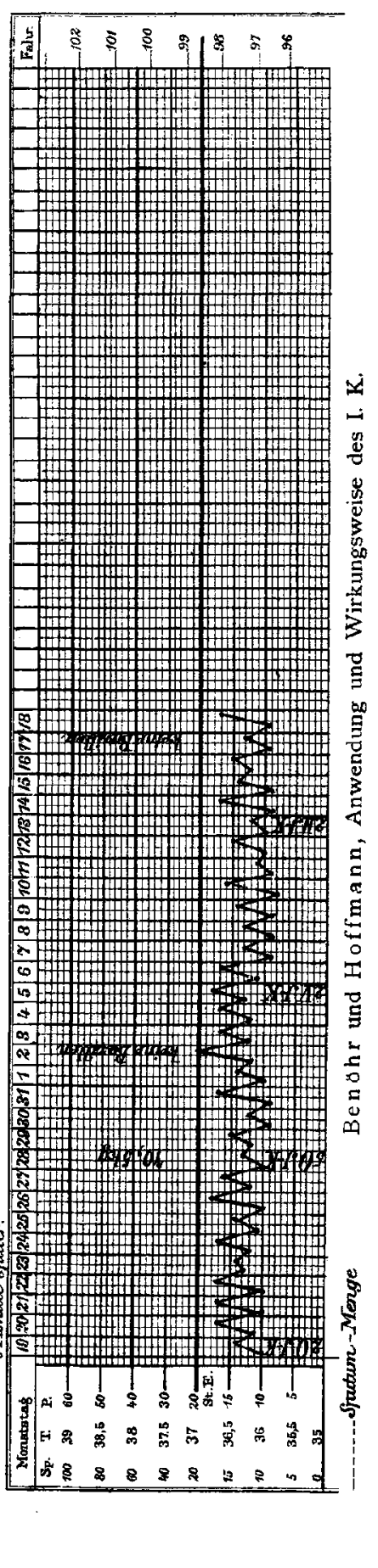




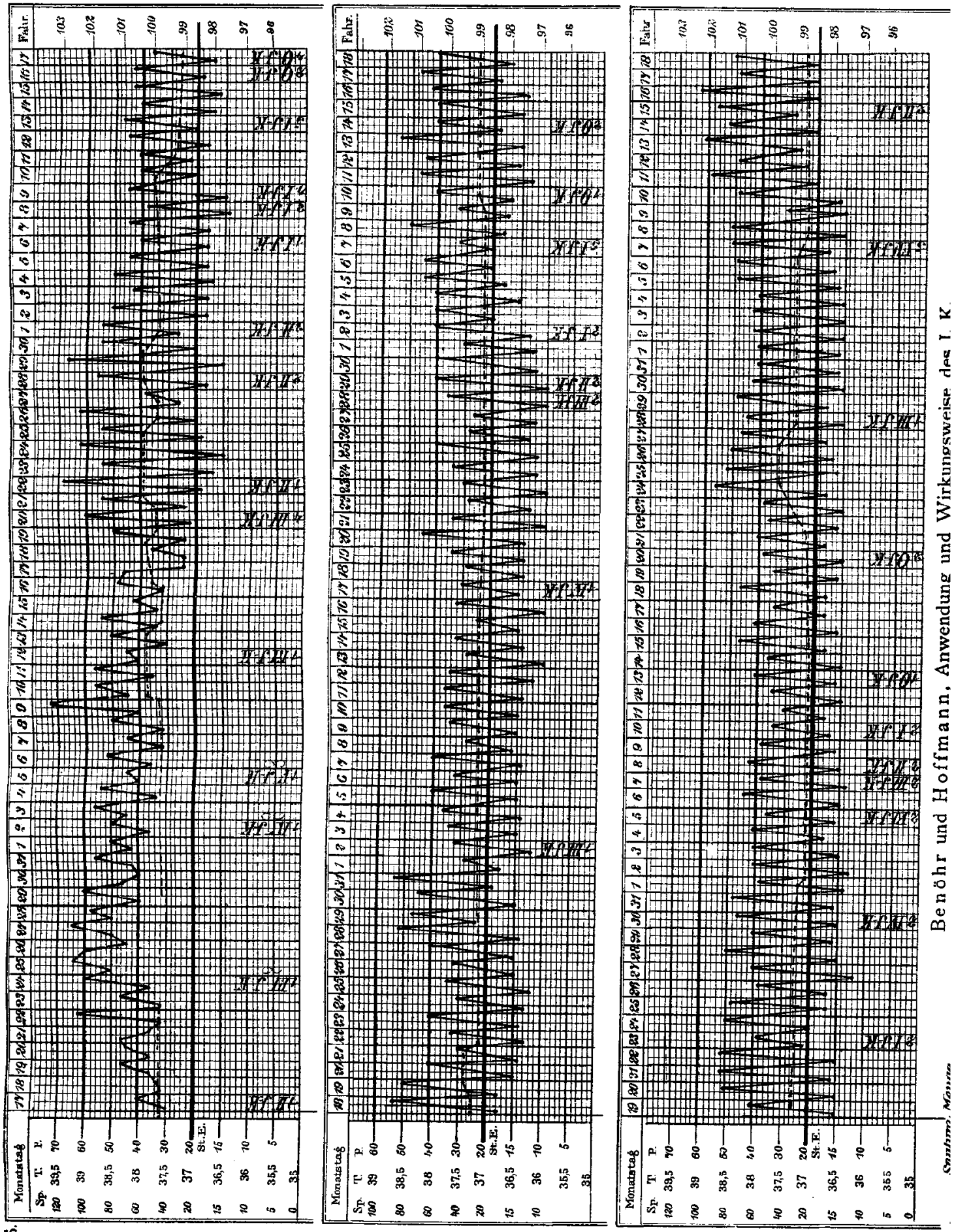



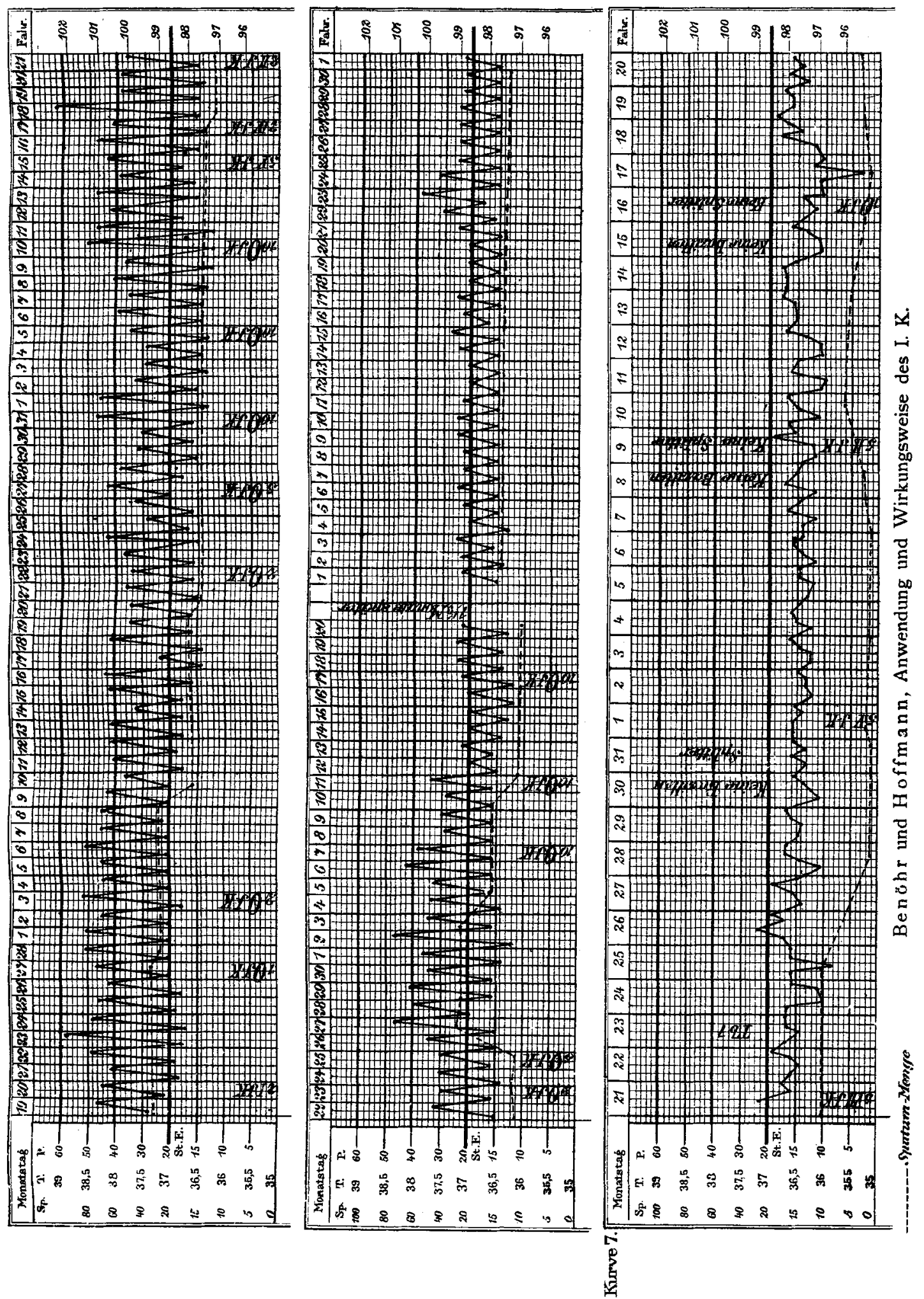

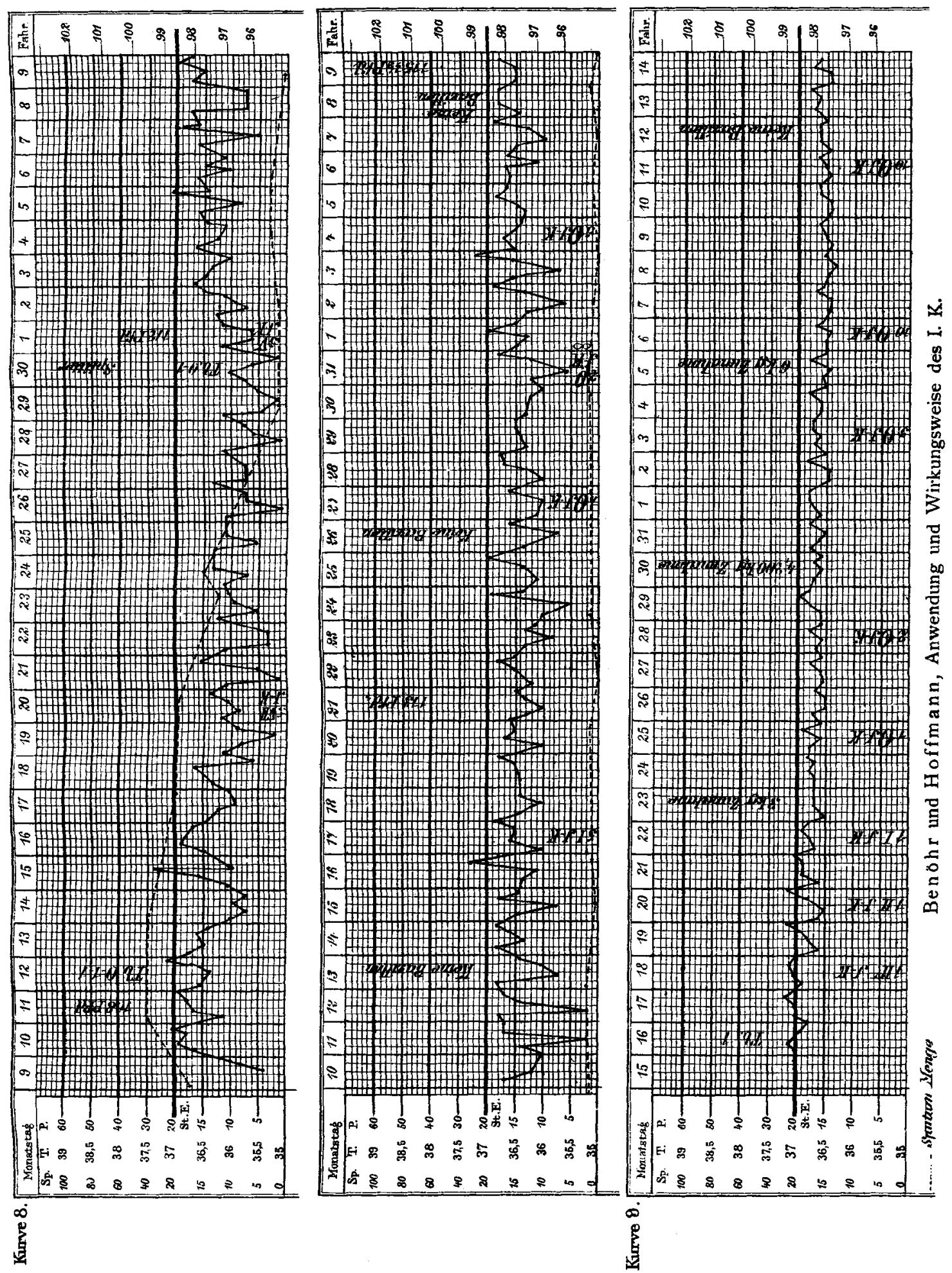

Curt Kabitzsch (A. Stuber's Verlag), Warzburg.

Kgl. Univ.-Druckerei H. Sturtz A. G., Worzburg. 


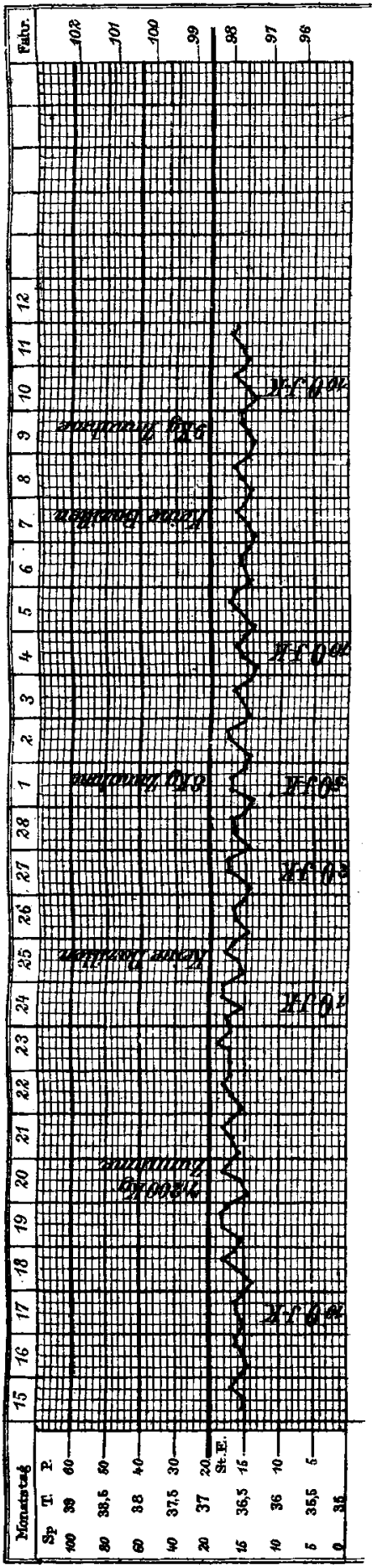

\% \$ \& : \$

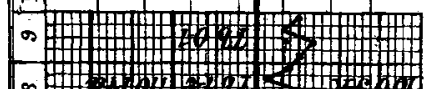
$\infty$ œ ऽ ‘̆ ‘s ๖`

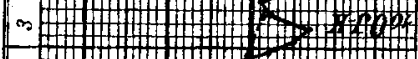
``冊 「r ஸे

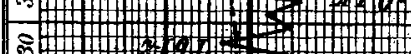
'̌̀ গ̊ํ

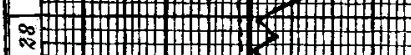

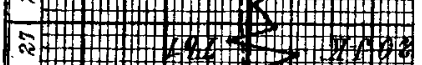
๖๖ 地 地

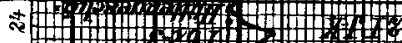

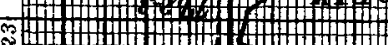
( 氺 屁 $\grave{\imath} \grave{\imath}$

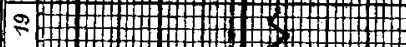

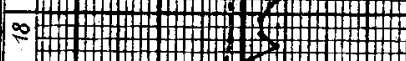
's $\stackrel{\imath}{\imath}$

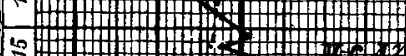
○̊ î́ 帘 ヘ̊ ₹ 尺

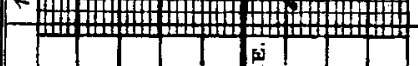

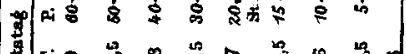
स\& 密

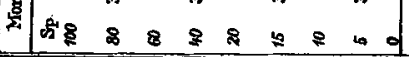
ㅇ 悬 $\sqrt{\text { \% }}$

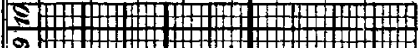
迥 vi ๖̊

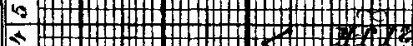

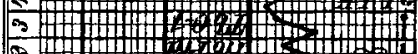
๖ 今 今 今 栬 地

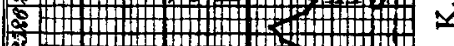
(十) वे P ⿷匚 进 亭 ,  次 今̌ 今 尺े ז 尺̌

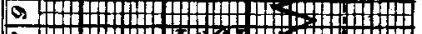
ळ , ì ๖っ 今心 今 今̊ 地 次地 (2) ¿ 今 壾 ¿̀ š פ̧

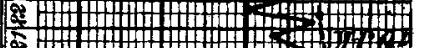
今, I (s र̌ 次 is

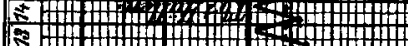
冷 ז I

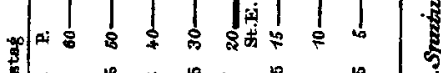
Fi 亮 


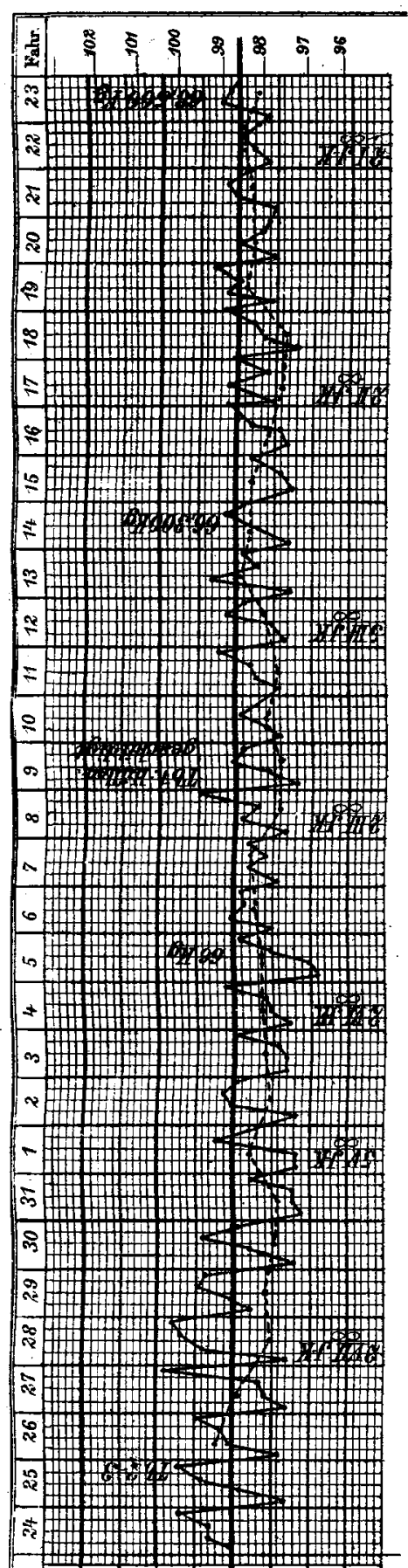$$
\text { J. }
$$

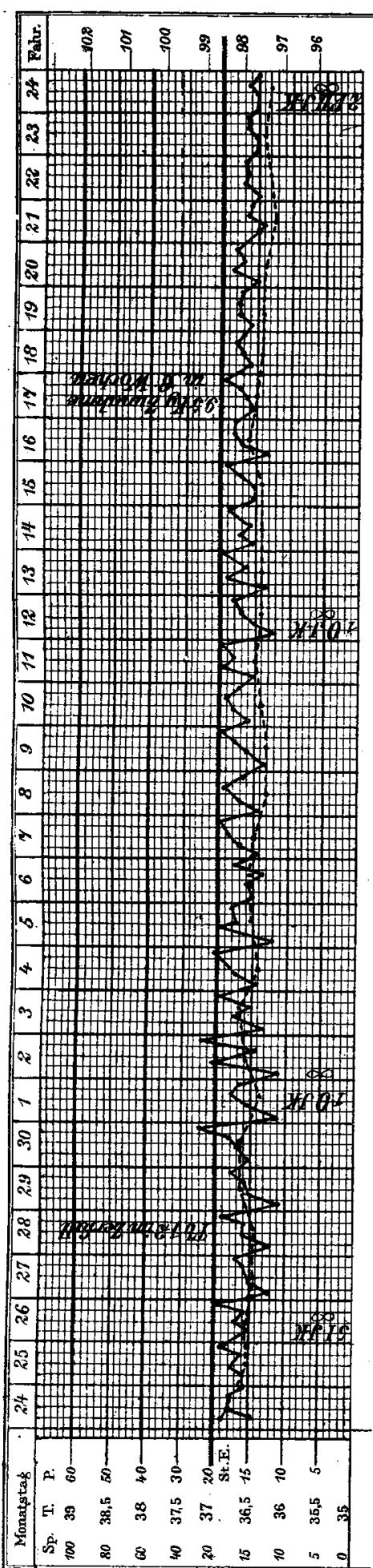

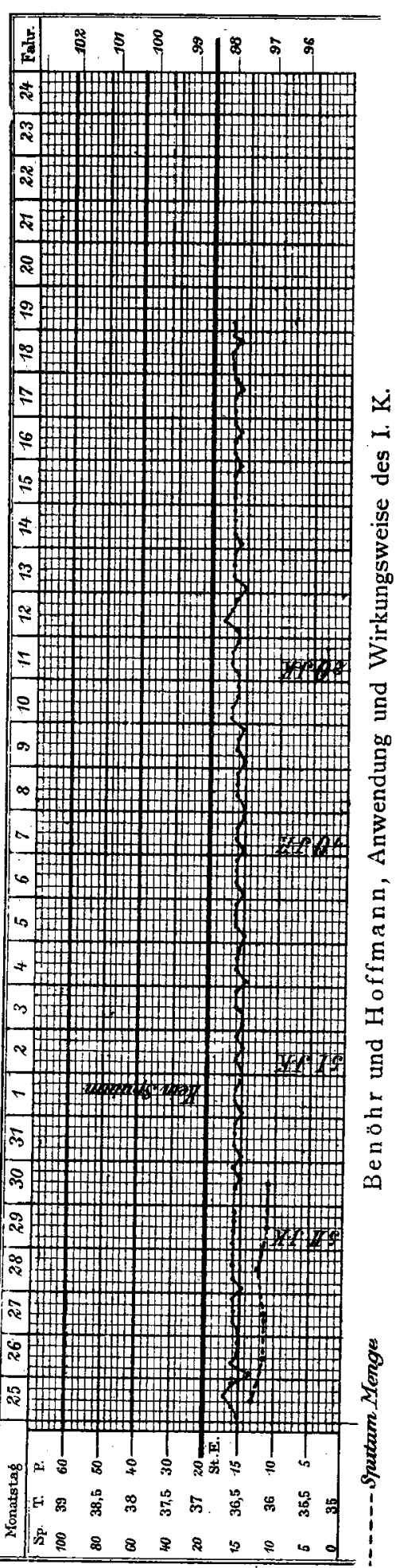

